



\title{
ANTHROPOCENTRIC: \\ Exploring real-time data for human centric architecture
}

\author{
BY
}

Timothy Graham Voss

A 120-point thesis

submitted to the Victoria University of Wellington in partial fulfilment of the requirements for the degree of Master of Interior Architecture

Victoria University of Wellington

School of Architecture 


\subsection{ABSTRACT}

This thesis explores applications of Mixed Reality, commonplace technologies and representation techniques in embodied and interactive design, through the development of an airport wayfinding system. The proposition that airports can be difficult to navigate, struggling to foster social connections, along with the challenging notion of providing an interface for Big Data spatially to users, motivates the research.

The development of personalised spatial wayfinding techniques aids methods for the use of location and big data to ergonomically and spatially represent users' navigation of space. Through methods of connecting people virtually within a single physical location using a unified design language, social implications of space are enhanced and extended. Finally, space which functions efficiency provides real-time feedback.

Key theory in Human Computer Interaction and Embodied Design informs the research, through mixed reality, technology and data-form translations.

Research is done over two stages, the first explores data inputs from users and represents these in $2 \mathrm{D}$ graphics. The second develops three separate design elements to create a spatial wayfinding system, to allow user engagement. These are a virtual projection, a set of physical forms and a set of wearable device applications. Design development happens through iterations within each experiment, and are always informed by previous work.

The result is an inhabitable data space with seamless embodied design exploring the localisation of large sets of data (fig. 0.01).

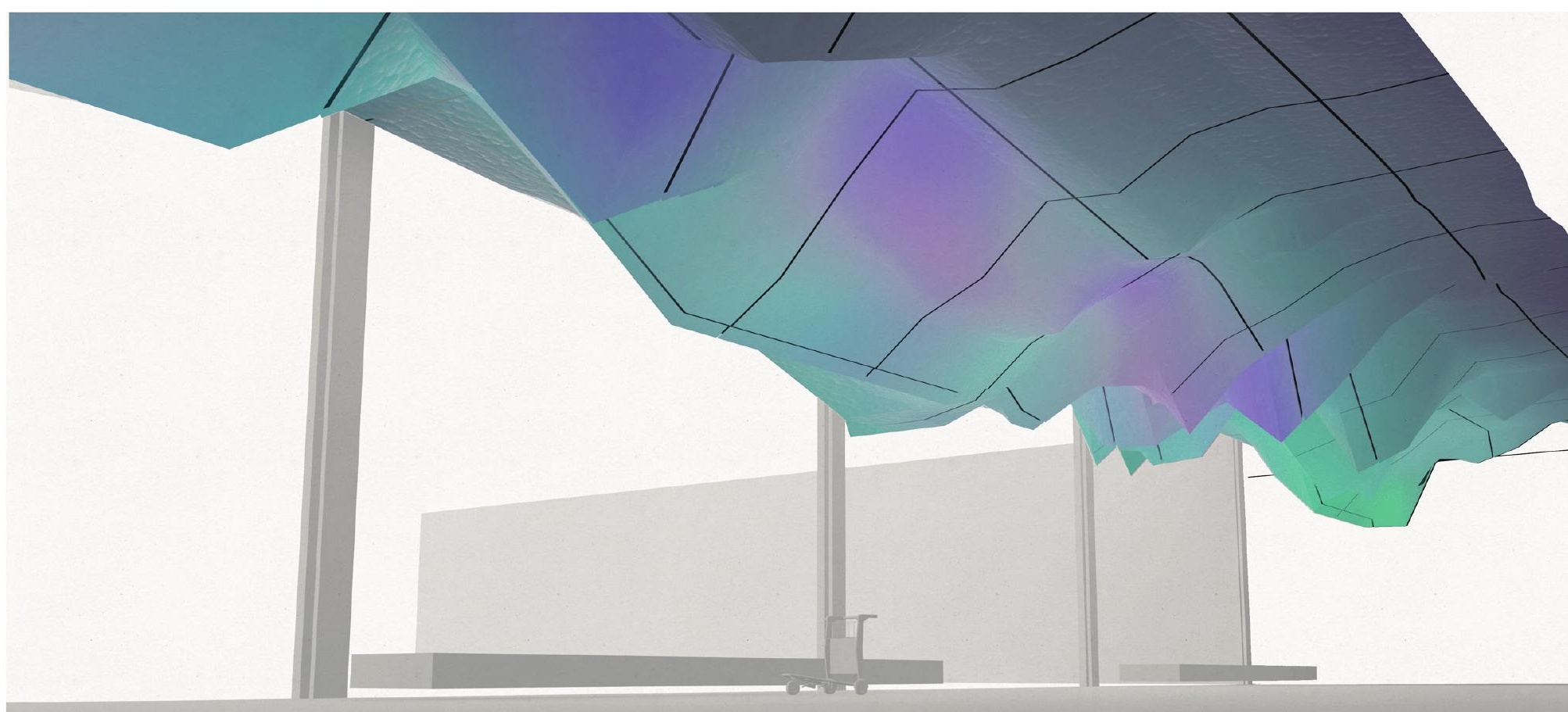

0.01 Design Solution - Virtual-Real space that provides real time way finding system situation within the context of an airport. 


\subsection{ACKNOWLEDGEMENTS}

I would like to thank my parents, Linda and Roger, for their tremendous amount of support over the past five years, encouraging me to aim high, and Helen Brunskill for her constant support.

Also my supervisor, Tane Motela, for his knowledge through the thesis year - encouraging me to explore new design processes and options.

Thank you to my colleagues in my stream, Julia Contessa, Tom Davies, Anneke Prins and Jared Zivkovic.

Also, thank you to reviewers through the year's critiques, Stephen Frith, Leonardo Carta, Kevin Sweet, John Hardwick-Smith, Euan Mac Kellar, Michael Spooner, Justine Clark, Nigel Groom and Simon Twose, for motivating feedback. 


\subsection{EXPERIMENT MAP}

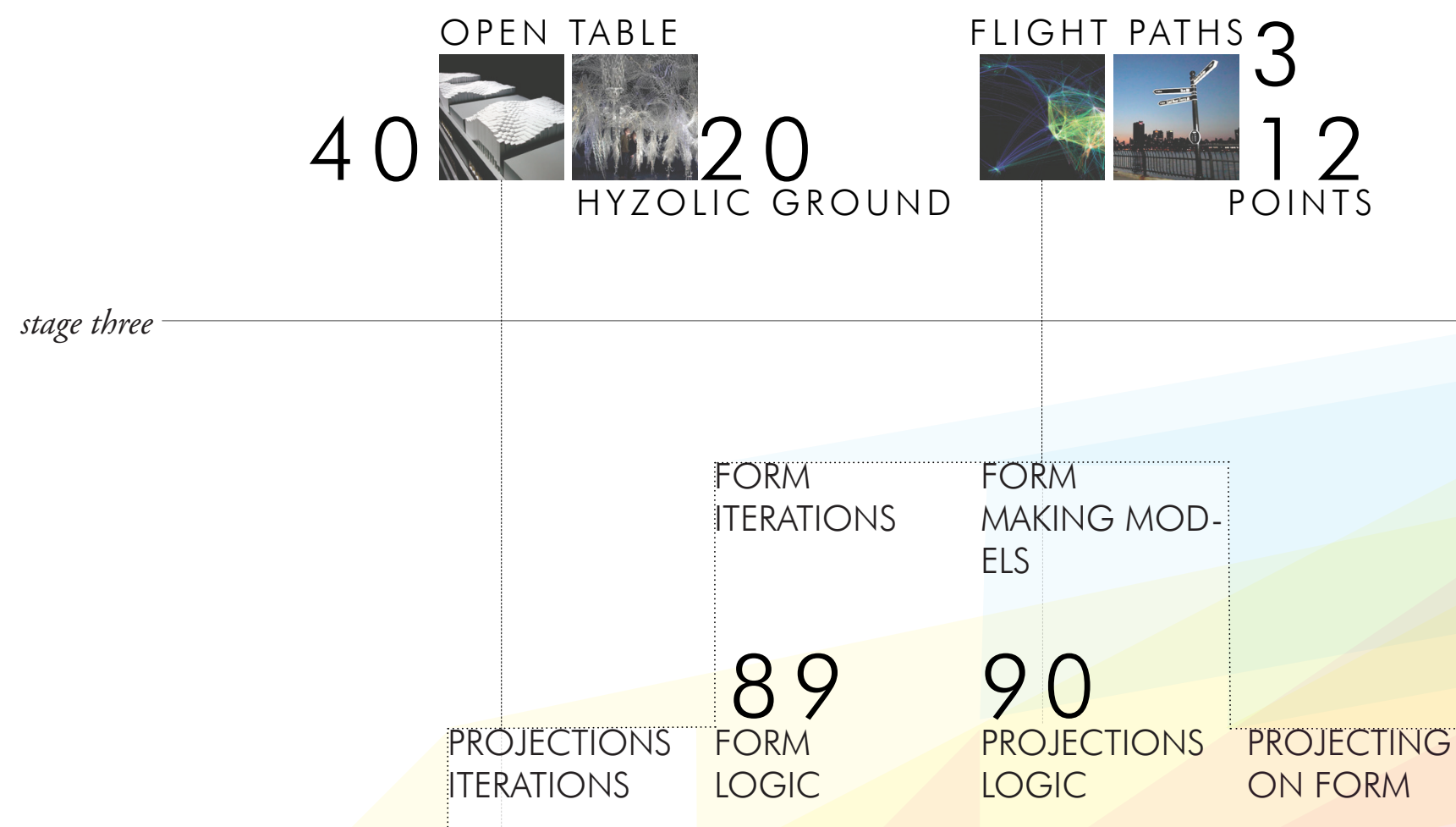

stage two

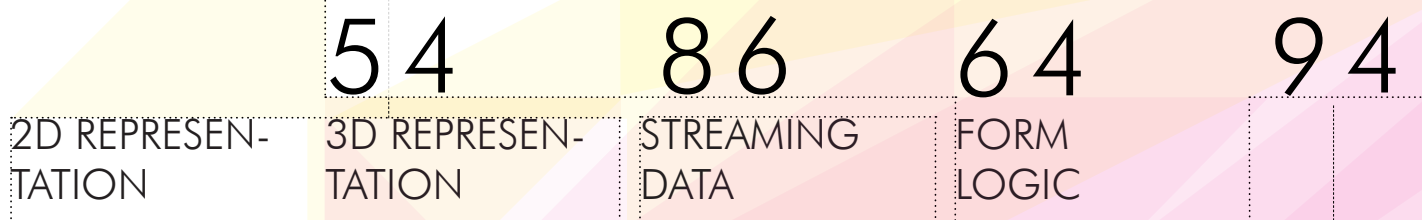

stage one

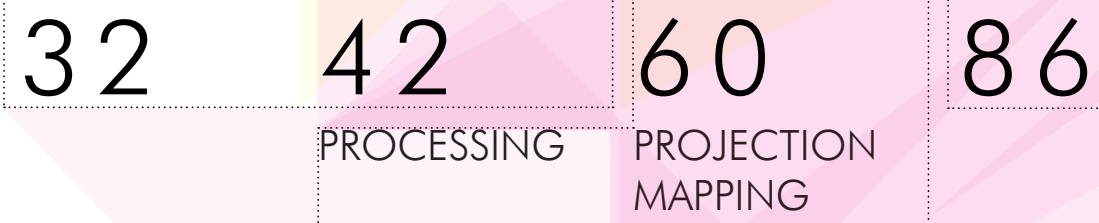

60

NETWORKING MOTION CAP-

TURE

technology tests

\section{8}

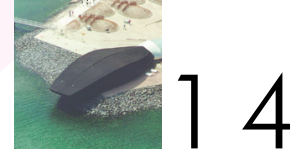

SALT WATER PAVILION

HUMAN

LOCATION 


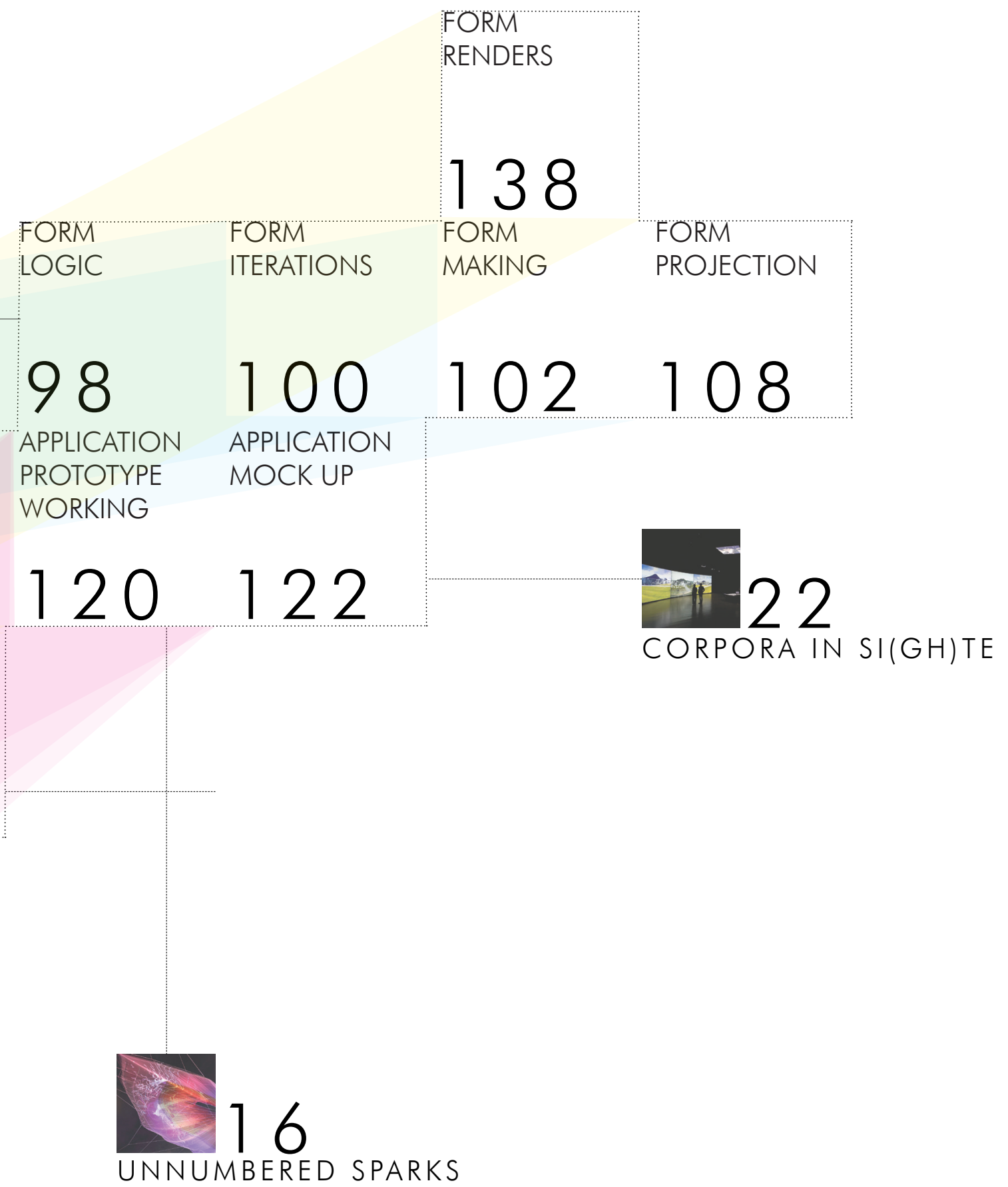

PROJECTION APPLICATION APPLICATION

INTERPRETA- PROTOTYPE MOCK UP

TION

76

PROJECTION

ON ARCHITEC-

TURE

68

UNNUMBERED SPARKS

0.02 The arrangement of experiments in relation to each other, with the locations of impact from precedent projects. 


\subsection{MOTIVATION}

The use of various software and technology in the context of architectural design is intriguing in today's fast moving digital culture. As technology filters into all aspects of life, it raises questions about how it can alter experiences and interaction within architecture. Interfaces and applications of software about space often don't have tangible links to the built environment, spatial or social experiences. The contrast between the building environment and digital displays can be jarring. By using architectural knowledge linked with pervasive technology, new spaces and interaction methods can be formed.

Just as software is used to draw and represent architecture, how can it also be custom-built to create a social architectural experience? The use of computation allows an extension of physical aspects to become digital and affect the use of space physically. This is what I aim to explore. 


\subsection{TABLE OF CONTENTS}

$\begin{array}{lr}\text { Abstract } & \text { iv } \\ \text { Acknowledgements } & \mathrm{v} \\ \text { Experiment Map } & \mathrm{vi} \\ \text { Motivation } & \text { ix } \\ \text { Table Of Contents } & \text { xi } \\ \text { SOCIAL, SPATIAL COMPUTING } & 1 \\ \text { Introduction } & 3 \\ \text { Theoretical Basis } & 4 \\ \text { Performance Criteria } & 6 \\ \text { process and Research Method } & 8 \\ \text { Precedent Analysis } & 10\end{array}$

REPRESENTING INPUTS $\quad 25$

Graphical 2D Representation 31

Responsive 3D Representation 42

Summary of Preliminary Design Stages 54

PERSONALISED WAY FINDING SYSTEM 57

Visualisations $\quad 60$

Mixed Reality $\quad 85$

$\begin{array}{ll}\text { Human Location } & 119\end{array}$

$\begin{array}{ll}\text { Resulting system } & 134\end{array}$

$\begin{array}{ll}\text { Summary } & 150\end{array}$

CONCLUSION \& CRITICAL REFLECTIONS 153

Conclusion $\quad 155$

Next Steps 157

Limitations 158

REFERENCES 159

References 161

Source of Figures $\quad 163$

Appendicies 165 


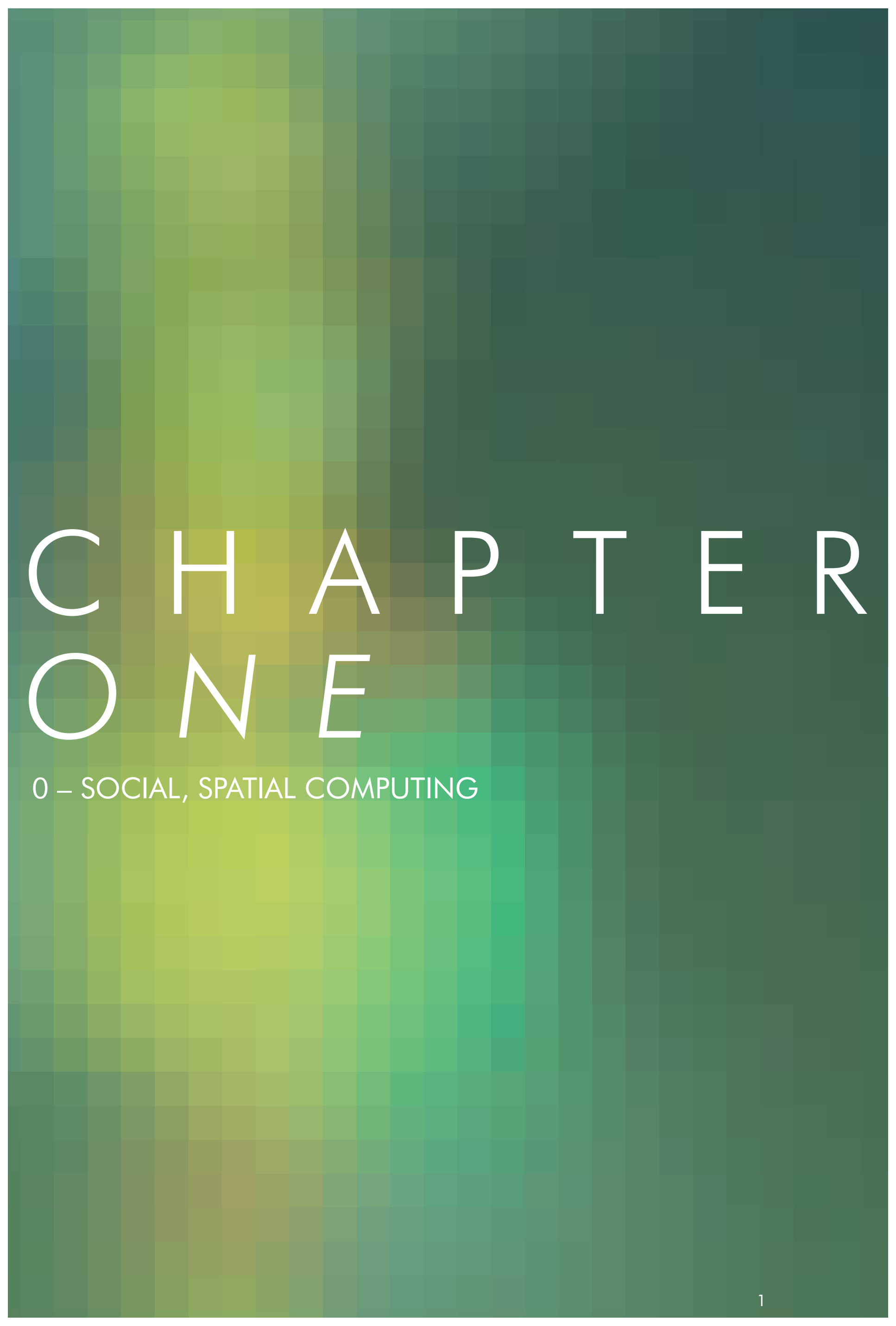




\subsection{INTRODUCTION}

Data flows are increasingly embedded in systems throughout society, facilitating the world around us. This 'big data' is rarely seen, accessed or understood directly by members of society. However, this data contains important trends that, if accessed, provide insights and cause implications beyond their

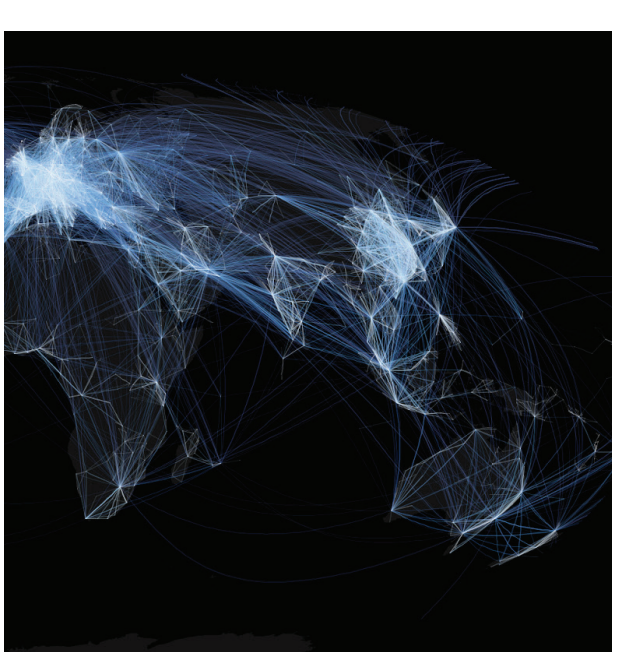

1.01 Flight Paths, by Aaron Koblin, is a representation of US flight paths into an interactive visualisation. Patterns are exposed through large amounts of data.

original use. Methods of accessing this data are usually done via dedicated computing devices - web browsers or applications.

Airports are filled with data, with thousands of people moving through them on a daily basis. Occupants depart or arrive on numerous flights, connecting locations around the world. All of these movements and flows are seen and displayed in data, such as Flight Paths (fig 1.01). However, these spaces do not often outwardly show the social constructs happening within, through their performance or experience. This spatial information can allow users to easily navigate space and encourage more meaningful social experiences (Izaki \& Helme, 2014).

The thesis aims to work towards a spatial way finding system that creates an appropriate social and informative experience for users. Interaction with architectural space is needed for users to influence spatial qualities. This will inform the creation of a socially responsive architecture. Data about users should have an ability to influence design outcomes. Also, the ability for users navigate space through data will be explored. Finally, to enhance the social experience of space, allowing users to connect easily through space will be worked on. By research through design, methods of understanding data sources will be learned to increase the representation of these data sources in physical space. This leads to the following research question:

\section{HOW CAN DATA BE USED TO ALTER ARCHITECTURE TO PRODUCE AN AUTHENTIC HUMAN CENTRIC EXPERIENCE AND TO FACILITATE A SOCIALLY RESPONSIVE SPACE?}

Through a series of experiments, technical and architectural difficulties are explored. Wellington Airport is used as a site for the development. The initial design stage explores data sources about users, both locally direct (from within the space and directly from the user, i.e. movement, location, physical direct interaction) and globally indirect (interpreted from the user, but still about the space pulled from the cloud, i.e. weather data), and transforms these in a way that allows it to help and benefit users. 


\subsection{THEORETICAL BASIS}

Three key areas are being researched in the process of this thesis; the use of mixed reality (MR), technology and data physical translation within the context of interactive architecture and embodied design environments. Embodied interaction, as described by Dourish (2001), is the "interaction with computer systems that occupy our world, a world of physical and social reality, exploit this fact in how they interact with us". Humans typically interact with computational devices through a desktop computer or personal computation device, however, pervasive computing is the term of computing embedded in everyday objects. Considering space as a device embedded with computational power that can be connected to, new social experiences begin to emerge from within data sources.

Anders' Designing Mixed Reality (2007) overviews the designing of realvirtual objects and spaces, and principals to design by. These principals show the importance of fluid relationships between the two, a constant understanding of the effects on physical and digital parts. These principles align well with the design aims, so are altered slightly to fit within these, providing a theoretical framework for the design process (fig.1.03). Ways of interacting with virtuality is over viewed in From Virtuality to Reality and Back (Schnabel, Wang, Seichter, \& Kvan, 2007), which encourages links between humans and computers.

Two types of technologies allow Human Computer Interaction with Mixed Reality; ubiquitous (McCullough, 2004) and emerging. Achten (2015), propose that existing recently developed technologies can be used in interactive architecture projects to enhance and simplify interaction methods within these. Broad topics include wearables, cloud computing and internet of things, giving spatial inputs for greater interaction and better understood outputs. Wearables are devices to monitor human information, such as location, heart rate or steps. Cloud computing is an 'out sourced network of computing devices' to which local computing can be transferred to. Internet of Things is a 'computational representation of a physical object so that they can be manipulated by virtual entities (Achten, 2015). Human location is important to the design of spatial systems about people, wearable devices ensure that pervasive computing can take place (McCullough, 2004). These technologies, as concluded by Achten (2015), are important to providing a 'technological layer' on which to build interactive architecture. Kuo, Lin, Shen and Jeng (2004) develop a method for spatial exploration, giving a "computer generated representation of reality in real time, providing applications for the digital translation of data to embodied interaction, aligning with the aims of the research.

As this design process is about using data flows, transforming them into space flows, a suitable methodology must be understood. Achten's (1997) methods transform inputs into 'generic representations', 'a certain set of entities' with 'a particular meaning". Forms are assigned a representation before the data source is identified. Wu and Clayton (2015) outline examples of methods to translate data into physical 3D objects representative of multiple variables, creating a tangible manifestation of the data. This is processed data and $3 \mathrm{D}$ printed, but the project aims to use real time data as the input, hence the output will also be real time. 
These theorists will be used to directly influence the research; inform discussion points and understand the best route for future design iterations.

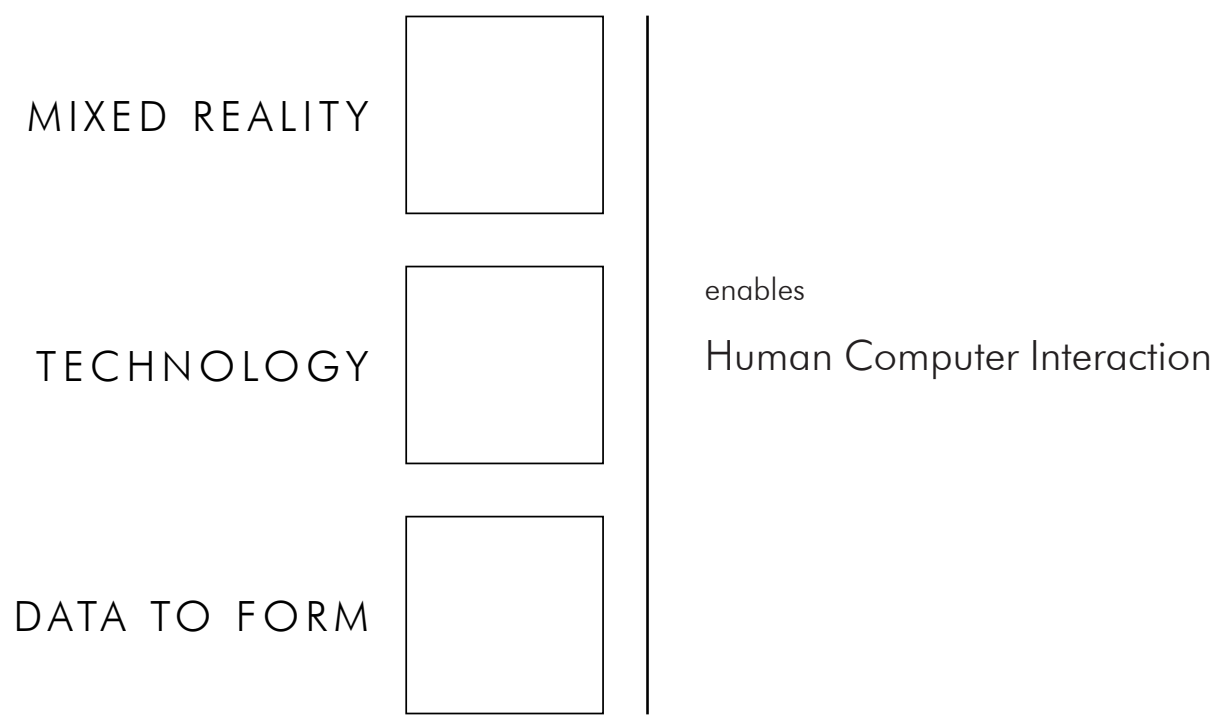




\subsection{PERFORMANCE CRITERIA}

The work done throughout the thesis is placed within Milgram and Kishino's (1994) Reality-Virtuality Continuum (fig. 1.03), between actuality and simulation, along with a selection Anders' (2007) principals of mixed reality design. This produces an index (fig. 1.02) on which to access outcomes. This index is chosen based on the research aims as mentioned below, to develop a unique solution.

They are the following:

- Comprehensive space between material, cognitive and symbolic

○ In creating MR space, these three ideals develop coherency and completeness. This ensures the space is meaningful, allowing information to be accessed.

- Corroboration and reciprocity between physical and virtual components

- That data influences spatial qualities, and the context of the space will influence the virtual representations of space. These should be equally considered and influence each other to become a single 'cybrid'.

- Extension of a coherent spatial experience and social context.

- The developed design extends social space, allowing an easier connection to other users.

- The project adheres to the context of the experience within.

- Anthropic Design (Anders, 2007)

○ The project is ergonomic and helpful to users, allowing them to navigate space easier. Human usability is be considered.

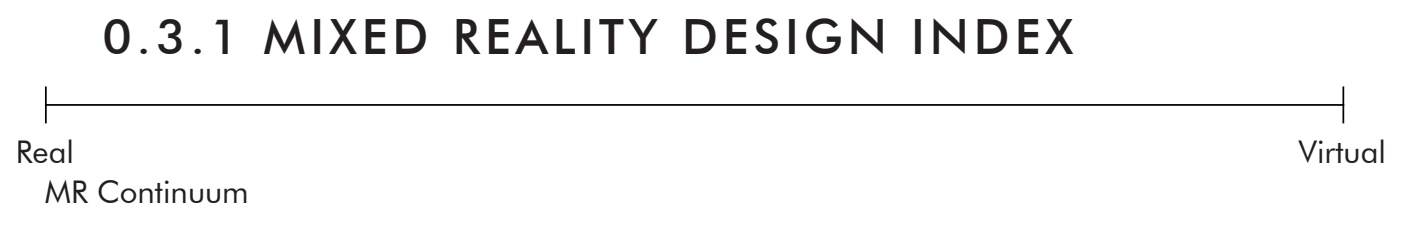

Comprehensive space between material, cognitive and symbolic

Corroboration and reciprocity between physical and virtual components

Extension of a coherent spatial experience and social context.

Anthropic Design

1.02 Assessment index to be used to determine the success of design experiments, in order to understand what aspects of the work should be improved on. Based off Milgram and Kishino (1994) and Anders (2007). 


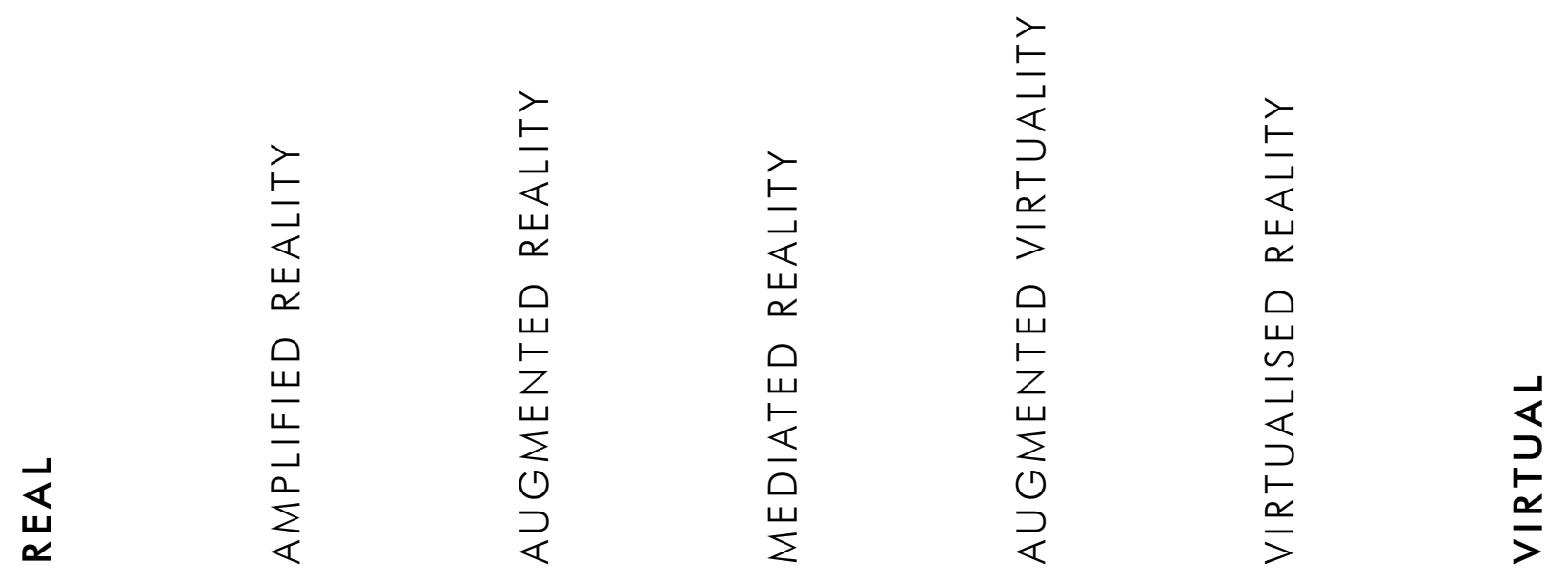

Salt Water Pavilion

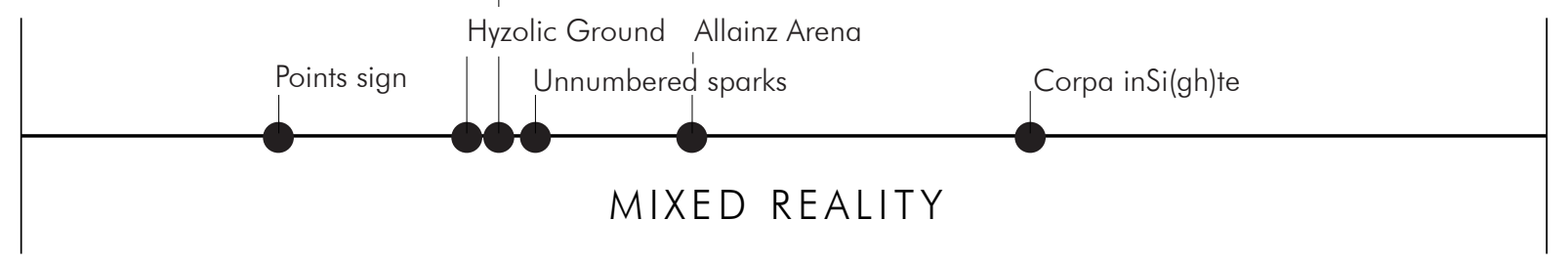

Augmented Reality

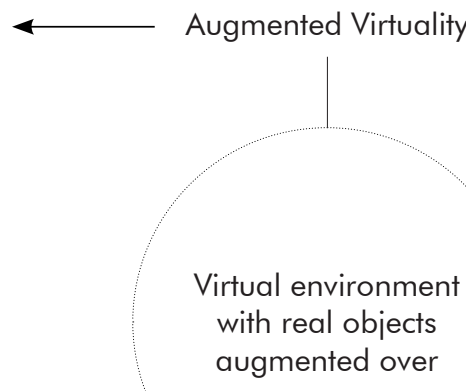

Real world environment

with computer

generated information

augmented over

with real objects

augmented over

1.03 Milgram and Kishino's Reality-Virtuality Continuum (1994), with descriptions of stages of composition between virtual and real. Precedent works are marked against this continuum, linking theory to practice works. 


\subsection{PROCESS AND RESEARCH METHOD}

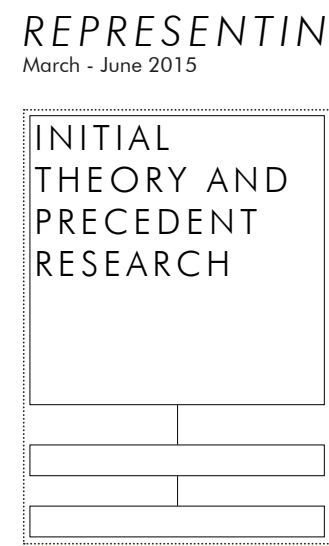

CH. 1

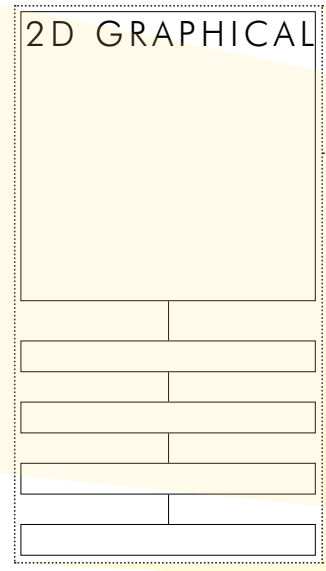

CH. 2.1

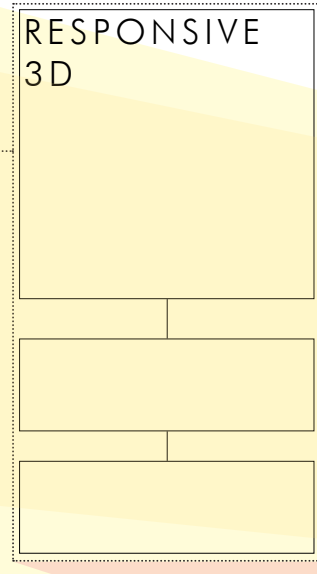

CH. 2.3

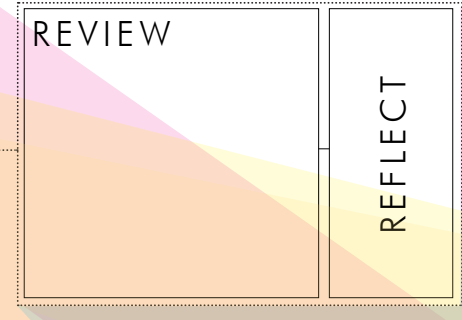

CH. 2.4

Design research can be broken into three types, research for, about and through design, linking together for a clear and considered research process (Downton, 2003).

Design through research is undertaken in three major parts (fig.1.04). Each step of an experiment involves the addition or layering of new information to better meet design goals and work to further and redefine aims. Through digital architecture design, a quick iteration process takes place allowing an additive design process. The production of the design outcomes is fast, although writing logical software and logic for the production is time consuming. Tweaks can be made for refinement during design production, through critical reflection.

Research for design provides information to support the design process (Downton, 2003), and is through project, theory and technology reviews. This constant input of relevant works and theory are first overviewed at the beginning, then referred back to throughout the design process, ensuring there is an appropriate level of understanding of research topics.

\subsubsection{DIAGRAMMATIC LANGUAGES}

The thesis uses two diagrammatic languages; circular forms describe and analyse work done, rectilinear forms document process workings. 
WAY-FINDING PART ONE

June- August 2015

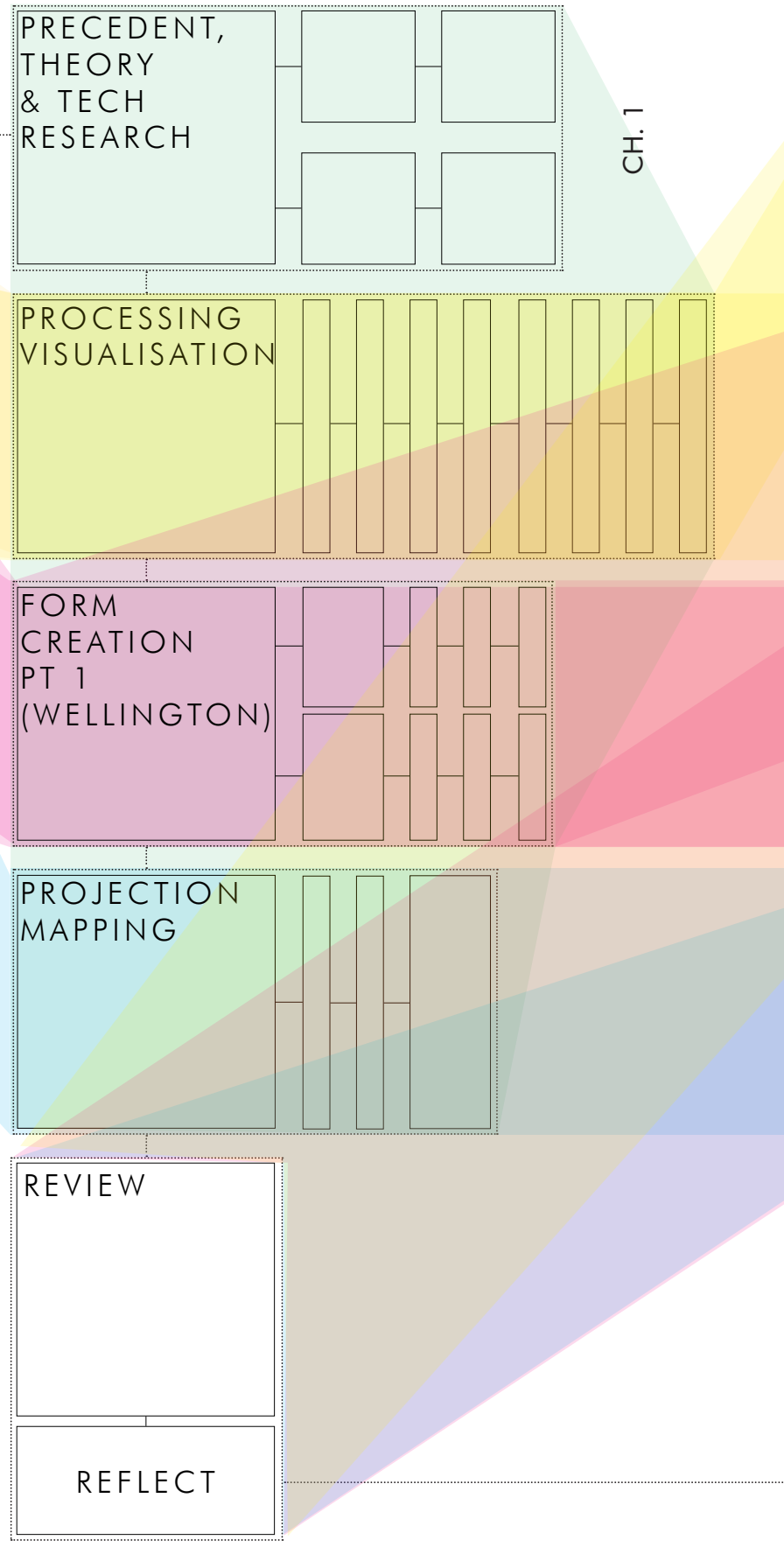

WAY-FINDING PART TWO

September - October 2015

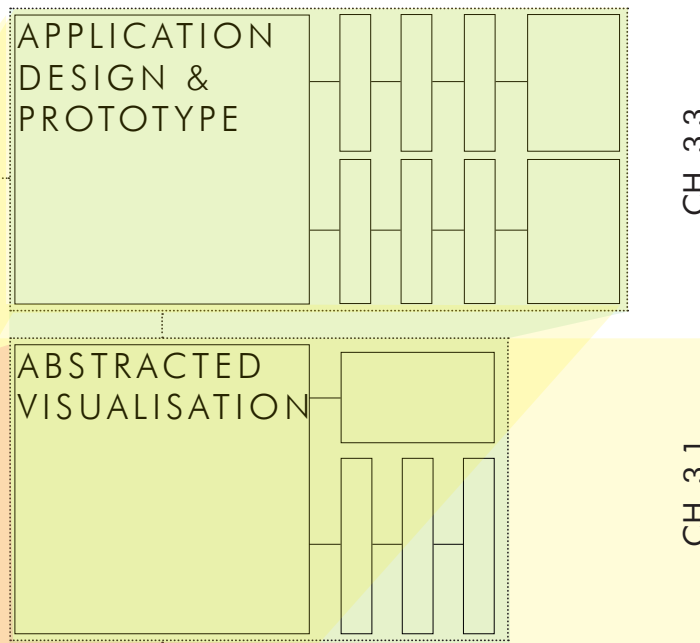

$\stackrel{m}{m}$
$\dot{I}$
$U$
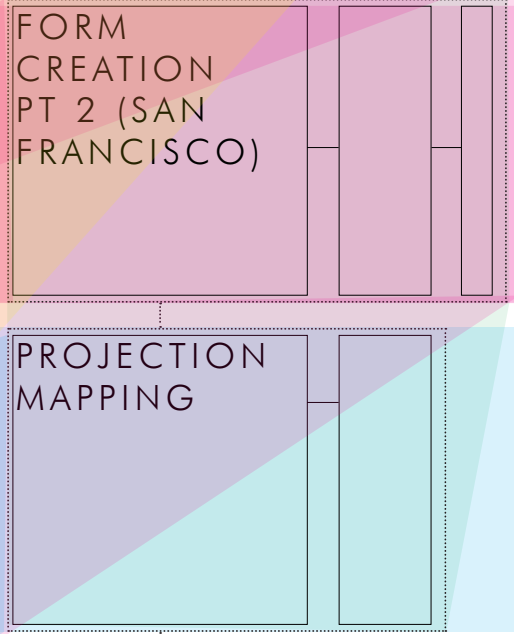

$\stackrel{\sim}{m}$

ড்

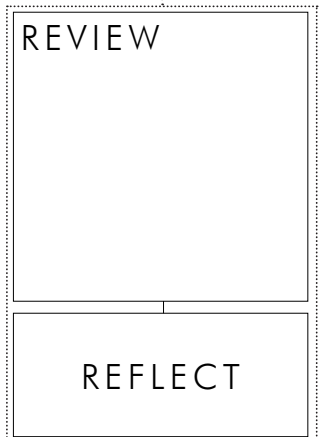

1.04 Process diagram across the three key stages of design. Project one had a different orientation to the wayfinding projects, but all three had corresponding themes. The process evolved and iterated each time it was done. The thesis is ordered in a similar way, however work from part two and three is grouped into experiment types to better show the evolution of the system as a whole. 


\subsection{PRECEDENT ANALYSIS}

These projects are used as case studies for the design experiments. They are chosen for their use of mixed reality, technology and spatial data representation techniques to create a responsive architecture around humans. Key theoretical influences are used to analyse these projects, and the ideal use of their technologies, aesthetics and systems in the creation of socially responsive airport experience is examined. 


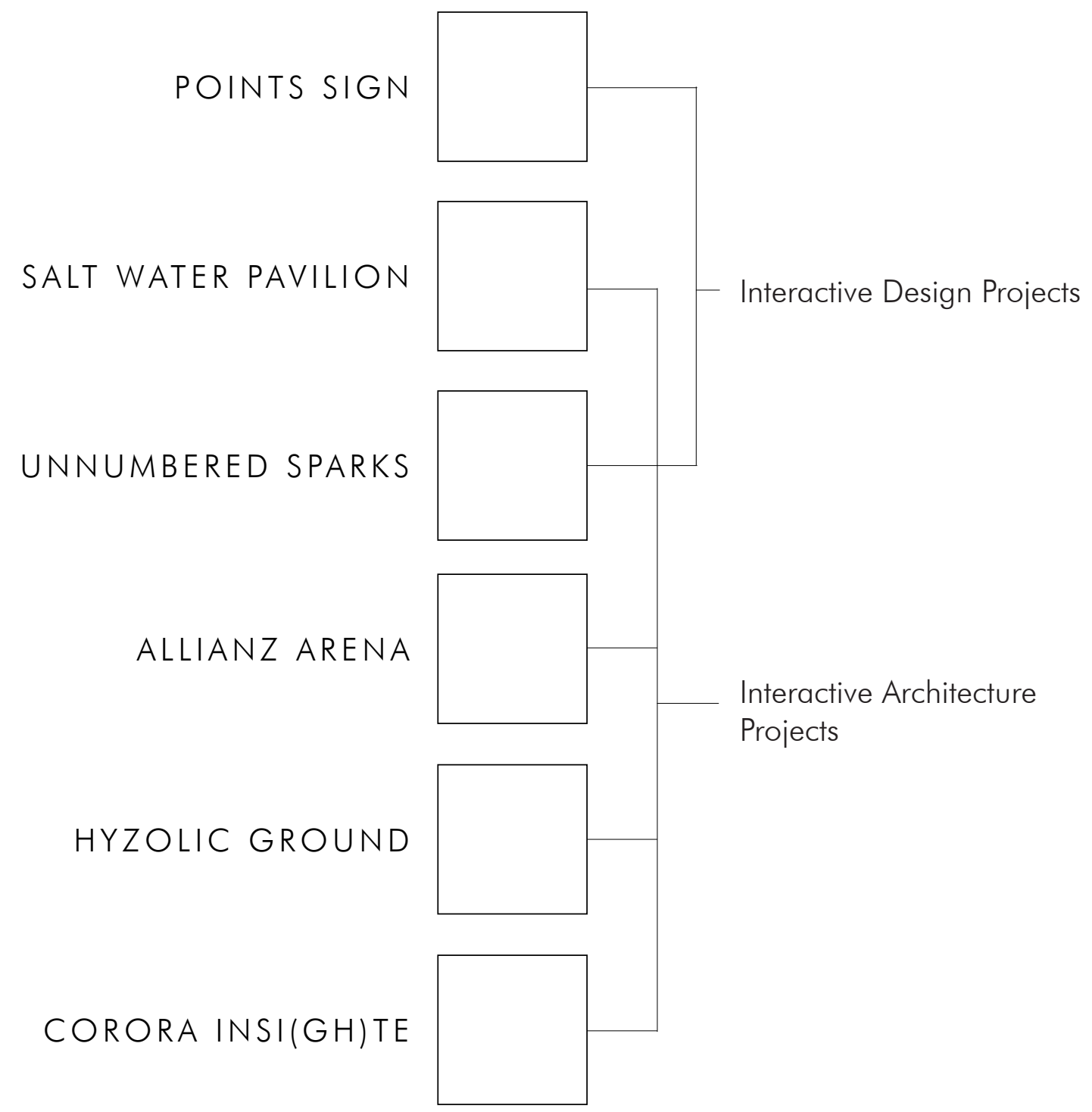




\section{5 .1 POINTS SIGN}

BREAKFAST

This project is a way finding system that links social networks and data driven API's, to humans, producing live and personalised information. The 'points' on the sign rotate as required with interactive buttons to allowing the sign to change information output (fig. 1.06). The data context means information given is never out of place, and uses data in a clear and refined way, showing methods of pulling large amounts of online data (fig.1.05), and producing an understandable amount of information for the users in real-time.

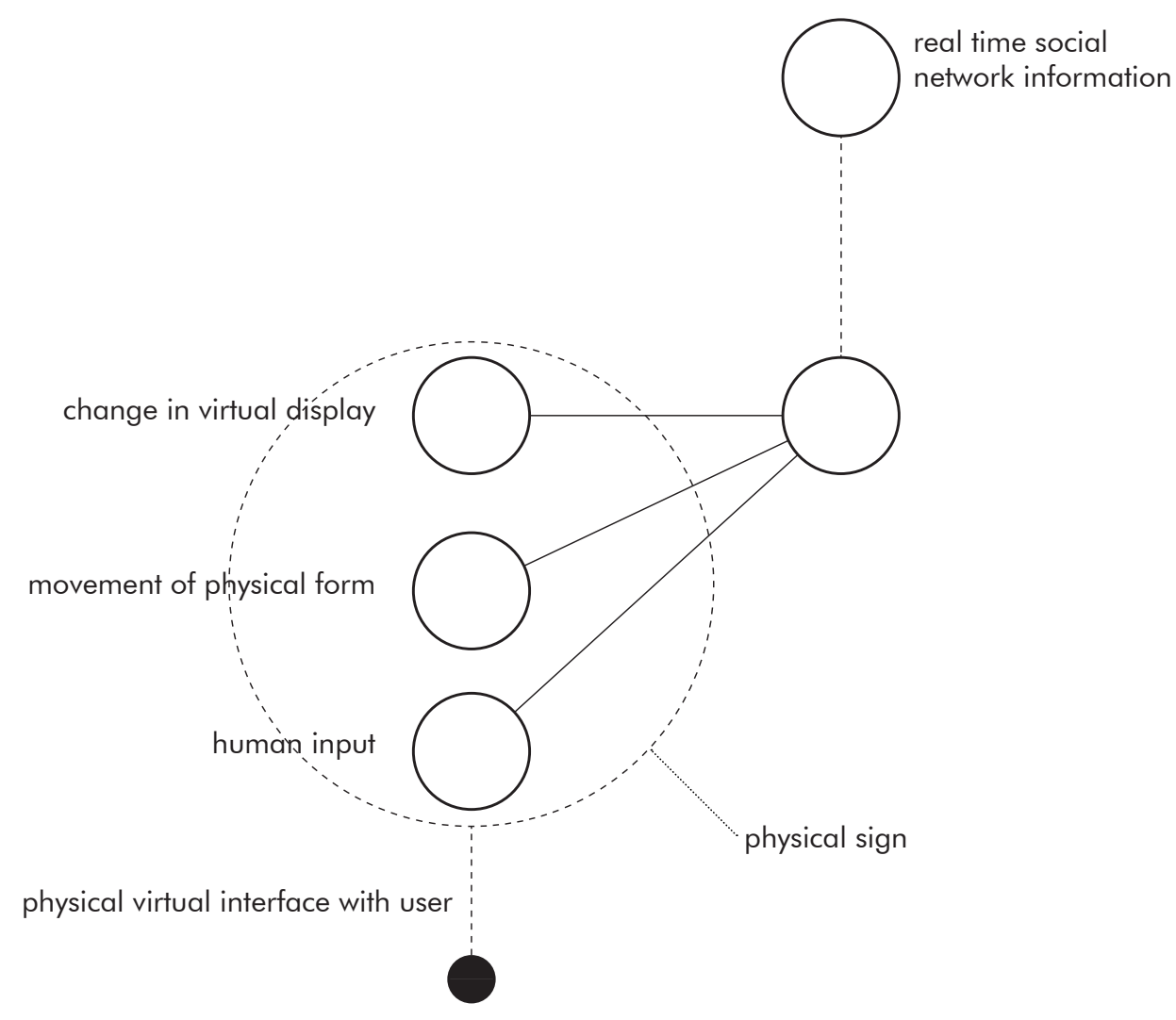

1.05 Combination of live inputs from social networking sources and direct user manipulation causes a unique, personalised context aware information display. Motorised pivots allow the directions to change as user's desire information. 

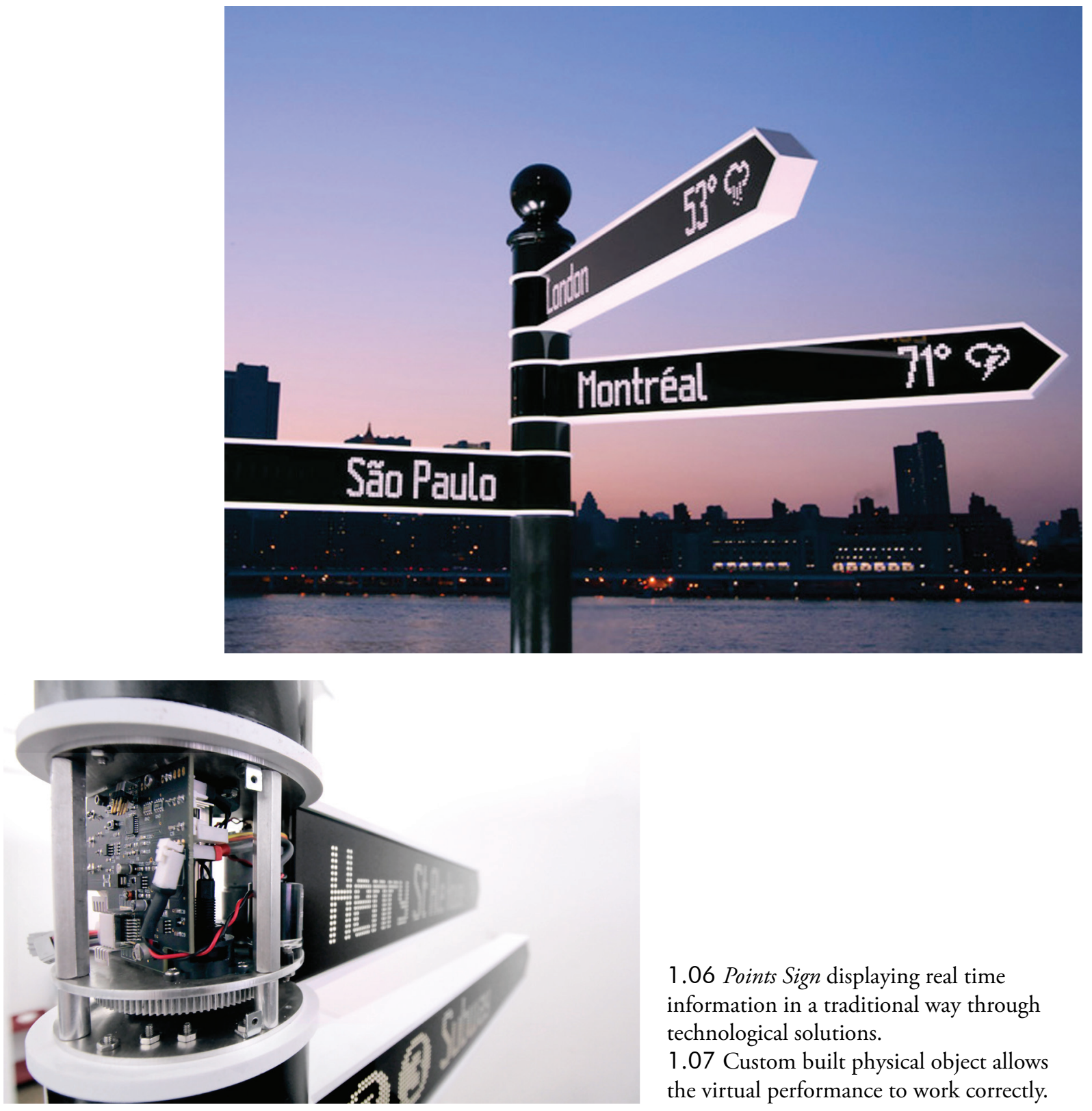

1.06 Points Sign displaying real time information in a traditional way through technological solutions.

1.07 Custom built physical object allows the virtual performance to work correctly. 


\subsubsection{SALT WATER PAVILION}

\section{ONL ARCHITECTURE}

Salt Water Pavilion is a sculptural form on the water's edge (fig. 1.09), a single element which moves using light and sound, rather than kinetics. Sensors and data feed weather information, allowing the architecture to constantly change itself and produce a sensory, multimedia physicaldigital experience. The users are mapped and tracked through the space, highlighting the importance for location models that feedback on themselves to create responsive architecture around both users and the environment (fig.1.08).

THE HYDRA KEEPS

FOLLOWING THE

VISITOR, SOMETIMES AS A STRUCTURAL ELEMENT, SOMETIMES AS AN INTERFACE, ALWAYS TRANSMITTING IN THE FORM OF SOUND AND LIGHT

-ONL ARCHITECTURE

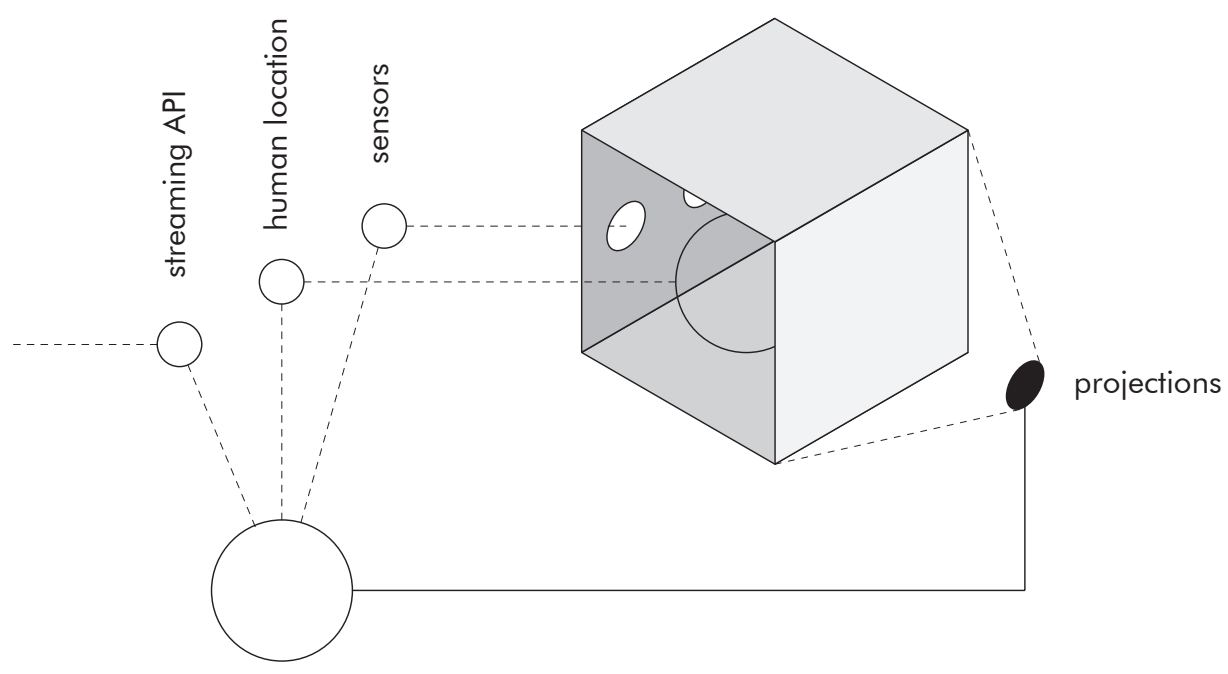

1.08 The architectural space allows information to be pulled from within. The human performance directly changes the spatial performance. 


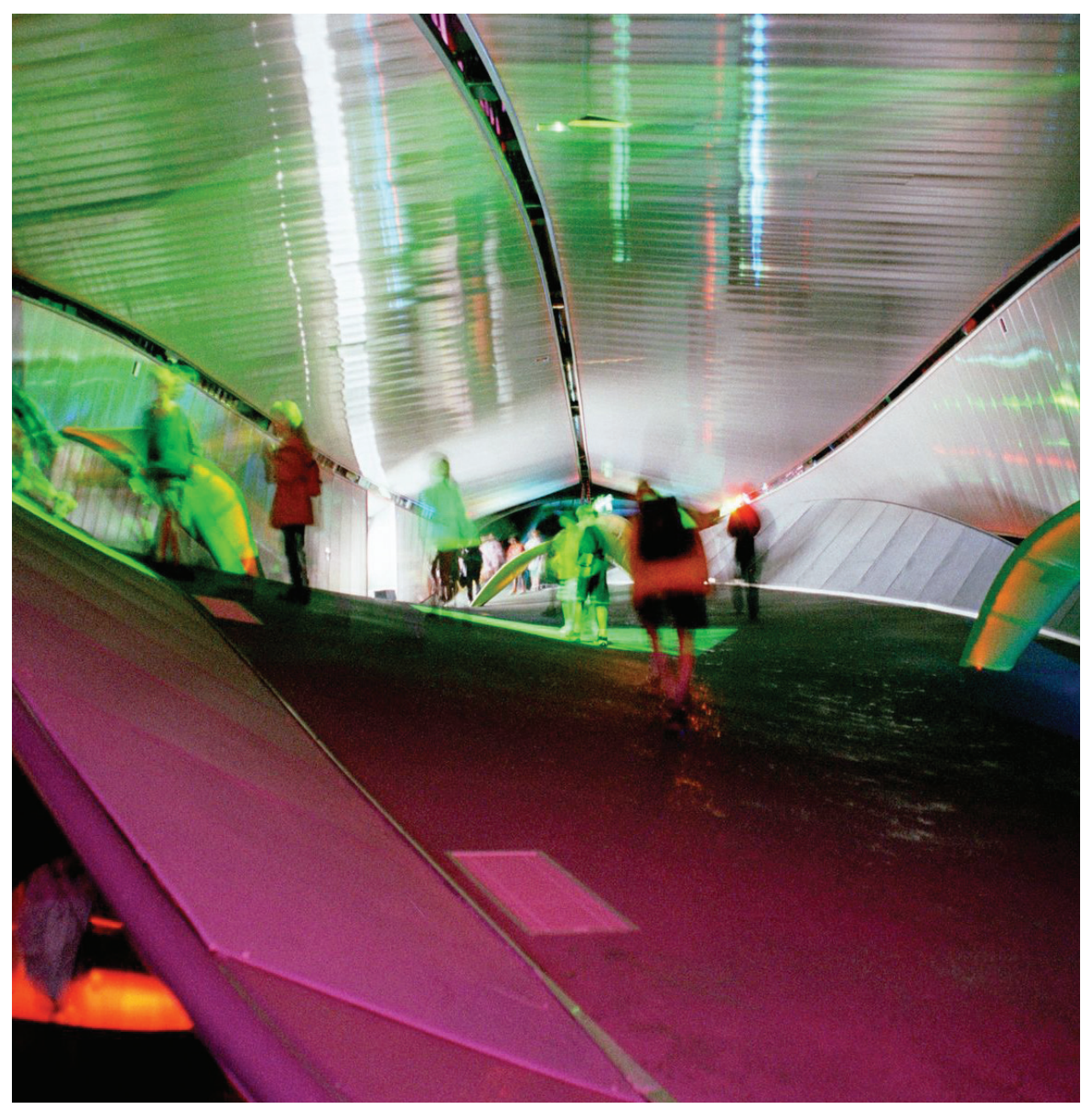

\subsection{Interior real-virtual form of}

projections. There is not a change in form, only the perceived form. The nonstandard geometry matches the irregular lighting with dynamic atmosphere to draw people through the space.

1.10 Exterior shell.

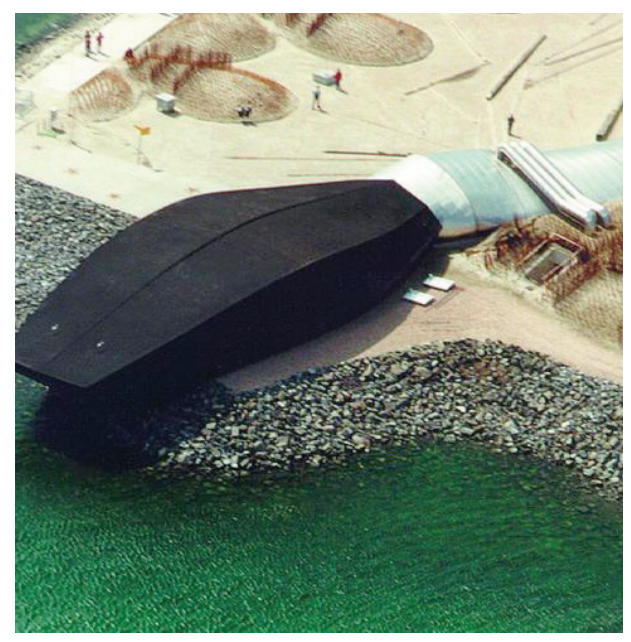




\subsubsection{UNNUMBERED SPARKS}

JANET ECHELMAN AND AARON KOBLIN

This project is an 'interactive community artwork' providing spatial experiences allowing users to digitally connect via smartphones (fig. 1.13). The installation is formed from a net like structure suspended between buildings which user manipulated projections can be displayed on.

The suspended spatial element can be seen by all, which creates atmosphere of the data projected onto it, is simple but effective. The controls use common technology allowing personal interaction with the space. The connections between users is via the digital projections, augmented onto reality (fig. 1.11).

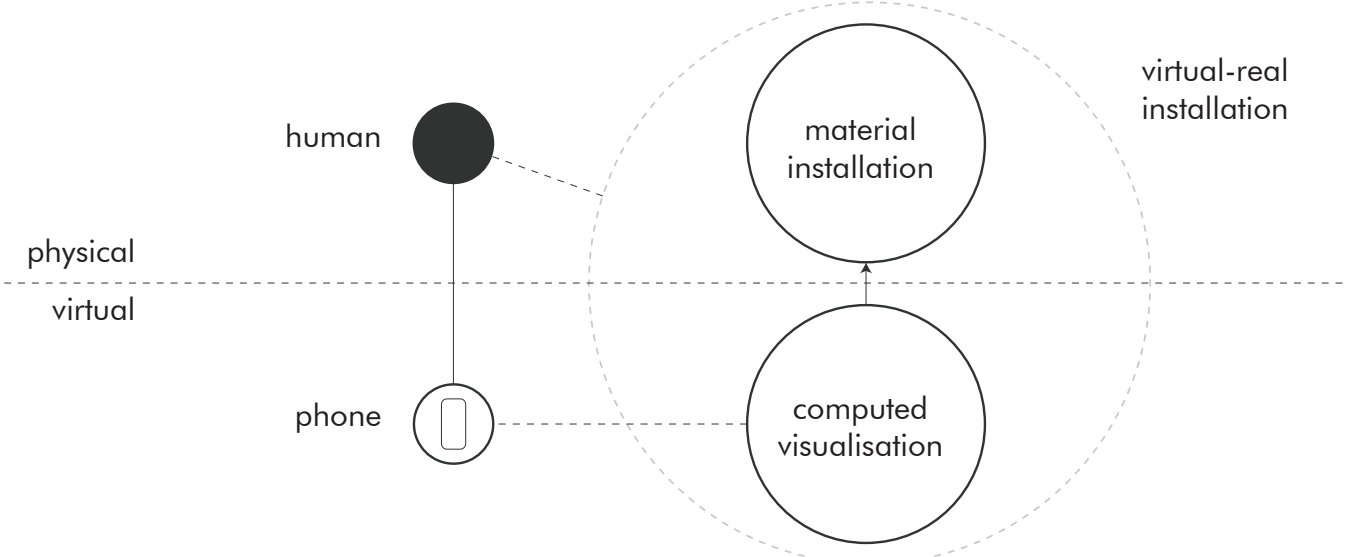

1.11 The combination of physical and digital for the installation creates something that is of both worlds. The suspended form's language likens itself to the virtual form through the use of sharp lines and mesh like outlines. 

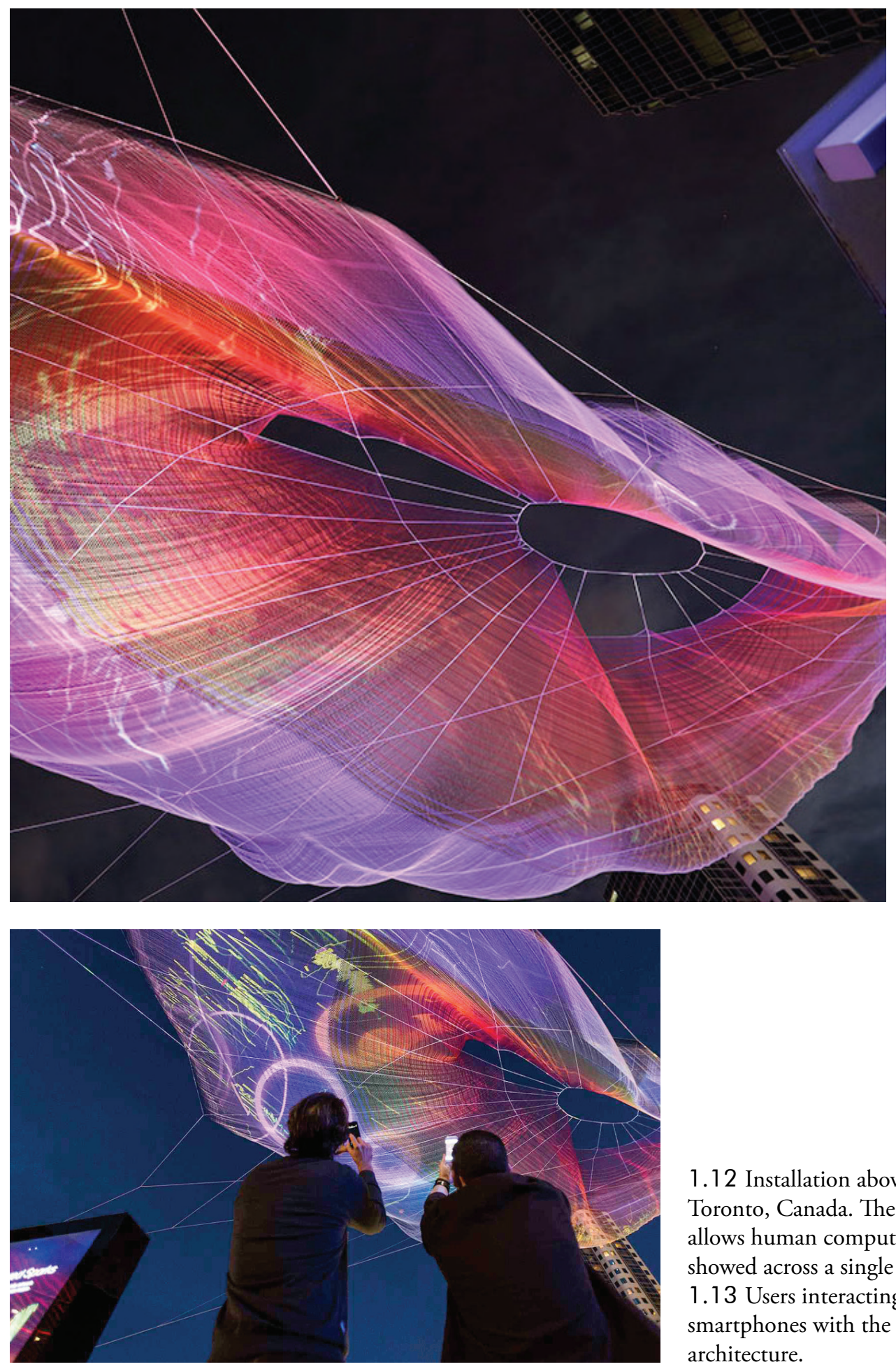

1.12 Installation above civic space in Toronto, Canada. The multiple user system allows human computer interaction to be showed across a single physical location. 1.13 Users interacting virtually through smartphones with the virtual/physical architecture. 


\subsubsection{ALLIANZ ARENA}

HERZOG \& DE MEURON

The football stadium features a reactive façade that changes appearance over time (fig. 1.15). Colours represent teams playing inside, of the activity happening within (fig. 1.14). The information is displayed through the illumination of ETFE cushions in patterns ("Herzog \& de Meuron - Allianz Areana," n.d.).

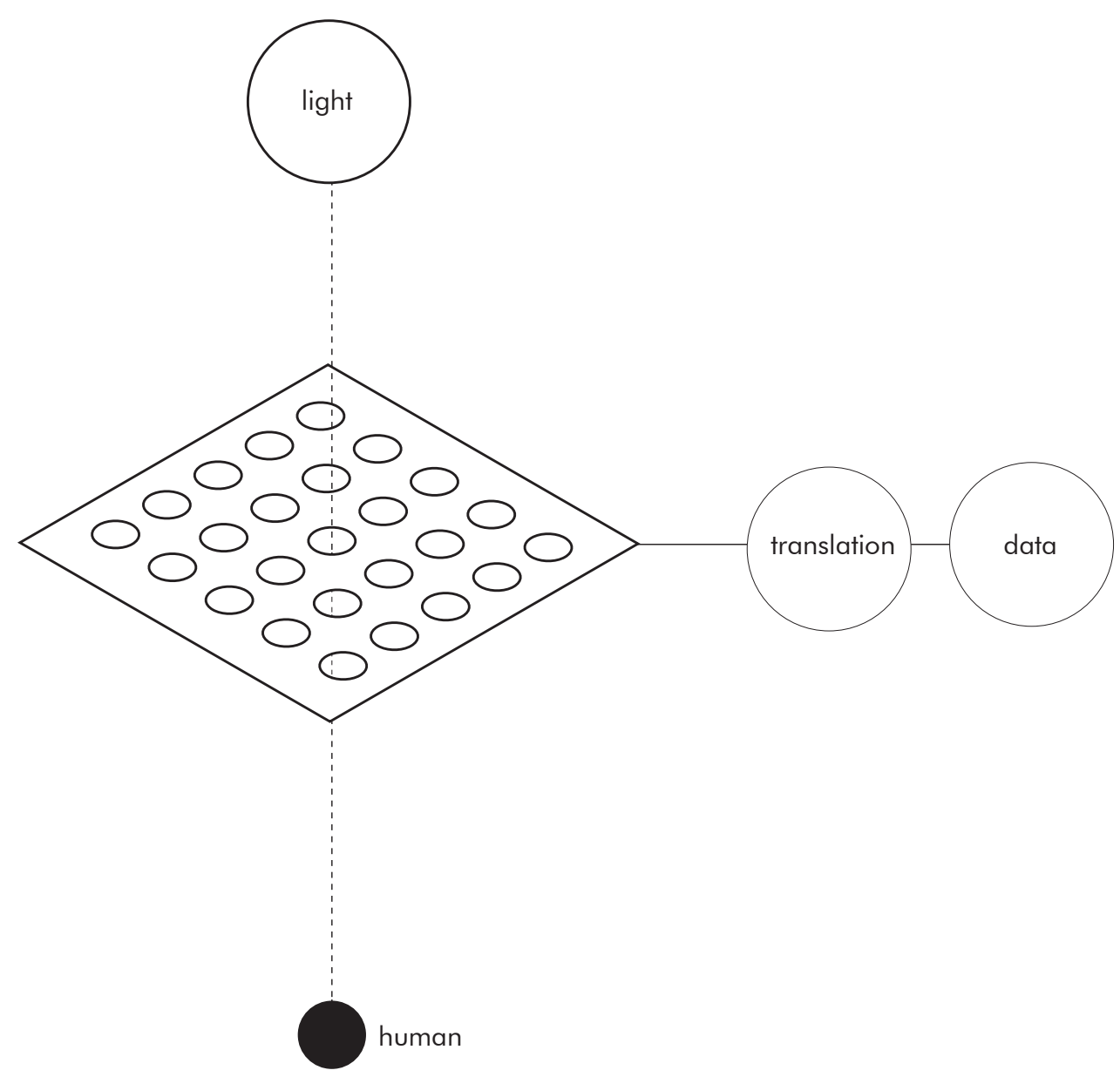

1.14 Using the façade system, the data could insert itself in the creation of space. 


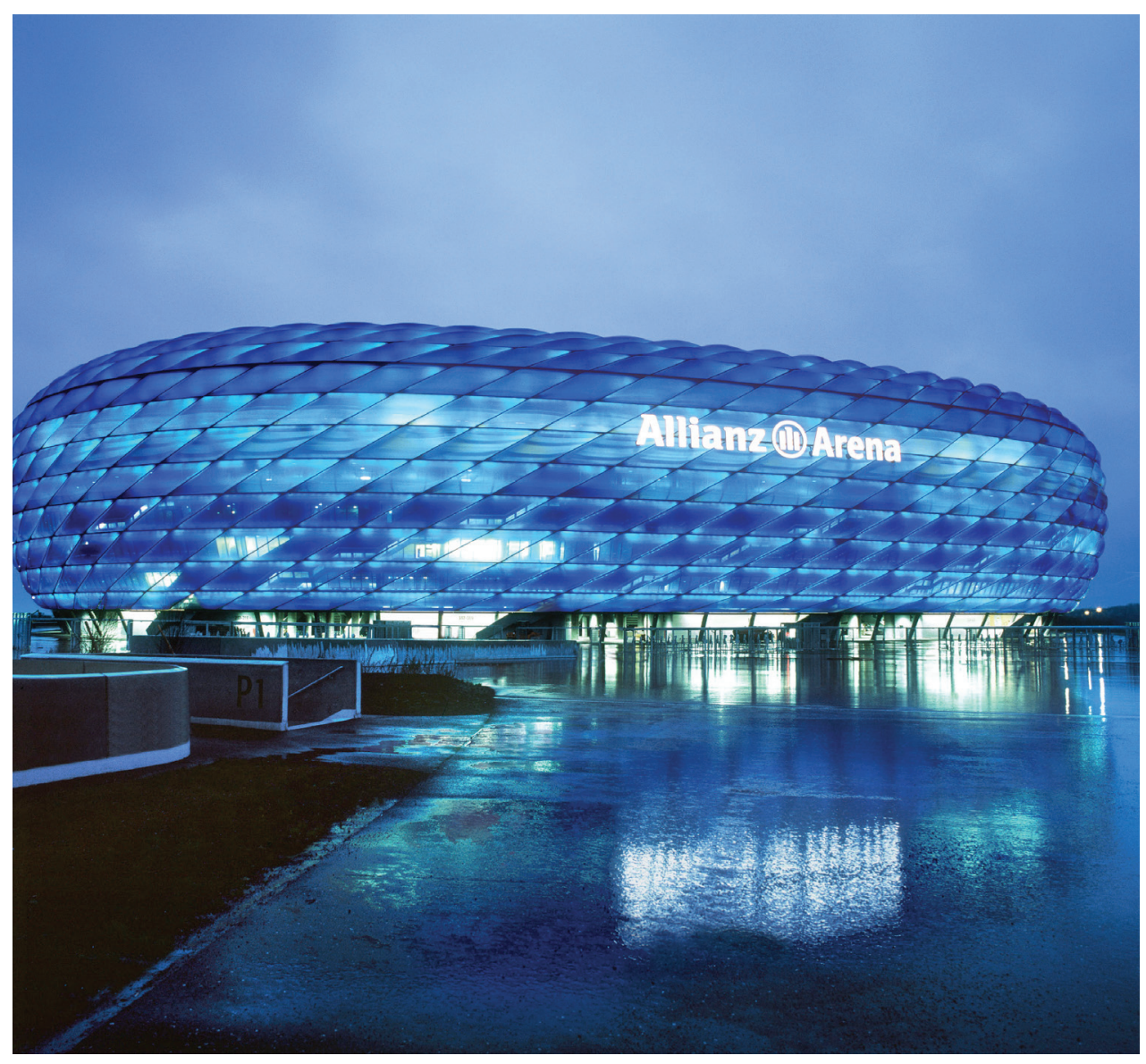

1.15 Altering atmosphere changes the way the space is perceived.

1.16 Overall exterior form of ETFE cushions.

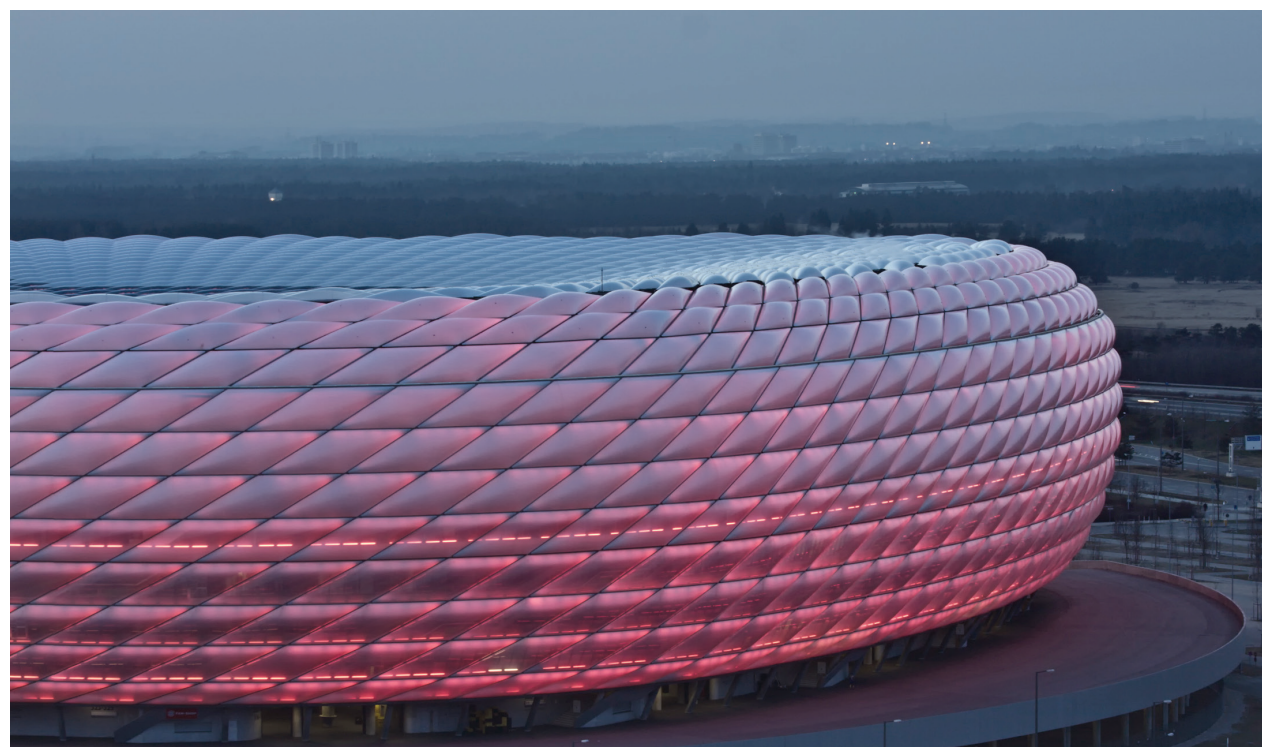




\subsubsection{HYZOLIC GROUND}

\section{PHILIP BEESLEY ARCHITECT}

This project uses sensors integrated throughout to alter the architectural form in real time. The project breathes with changes in the immediate environment it is within (fig. 1.18). The project then becomes a virtual - physical experience between the digital sensors and mechanisms and the physical form it is altering. This compounding creates a unified experience as one design. Sensors include motion tracking, touch and temperature and outputs to move and light the space through multiple human senses (fig. 1.17). The interaction method is taught by experimenting with in, there is no devices or standard form of computation present. Natural movement is displayed through computation, the output translates the environmental levels of temperature, sound etc. into architectural and spatial qualities.

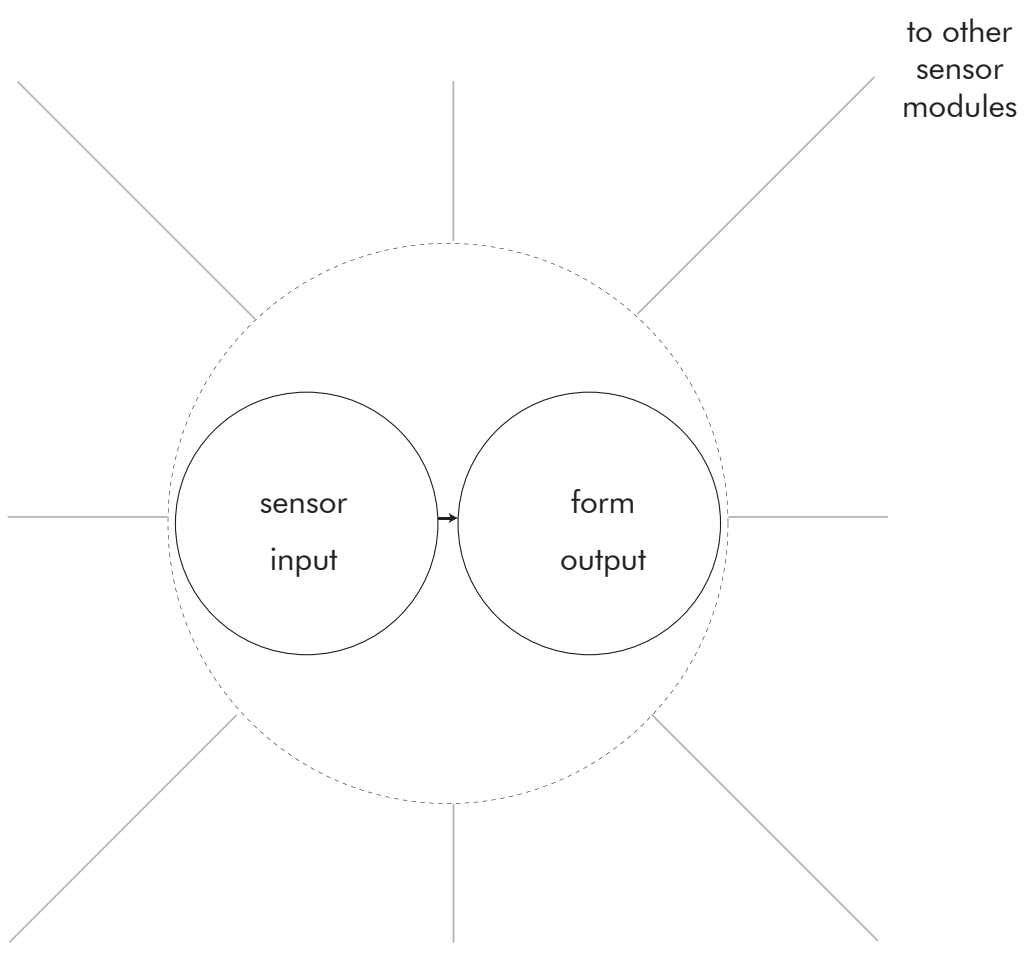

1.17 The architecture is turned into components both physically and digitally, acting as a multi agent system. 

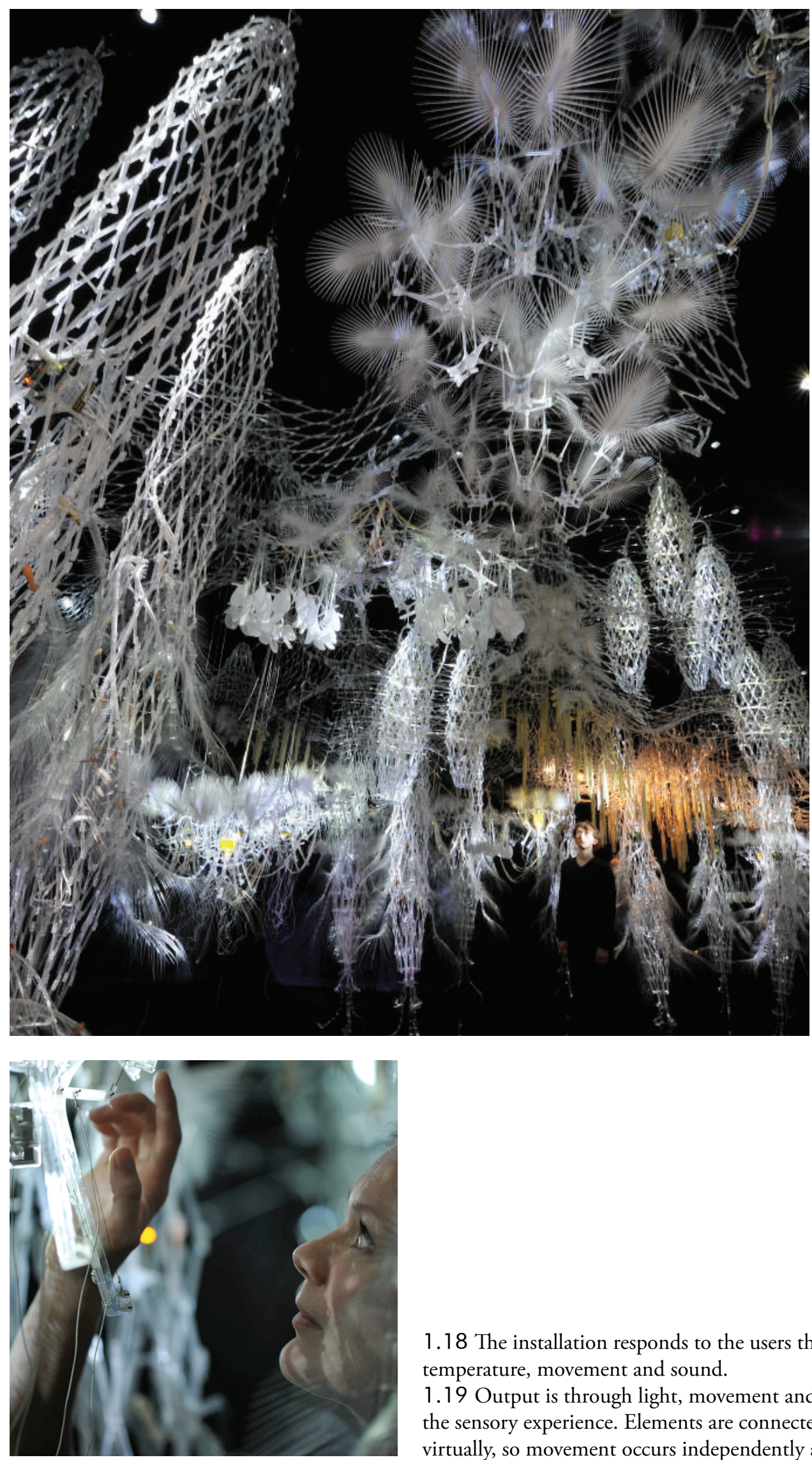

1.18 The installation responds to the users through sensors for temperature, movement and sound.

1.19 Output is through light, movement and smell, adding to the sensory experience. Elements are connected physically but not virtually, so movement occurs independently acting like cells. 


\subsubsection{CORPORA IN SI(GH)TE}

DOUBLENEGITAVES ARCHITECTURE

Using sensors on site, the architecture uses the Internet of Things approach to retrieve data and create real time virtual meshes of architecture, which change as the physical environment changes. The architecture is overlaid on the site. This link is important in understanding the inputs and outputs of a physical, virtual system, the way in which physical data is computed in real time and presented back to users (fig. 1.21). The network of sensors create an awareness of the site (fig. 1.20).

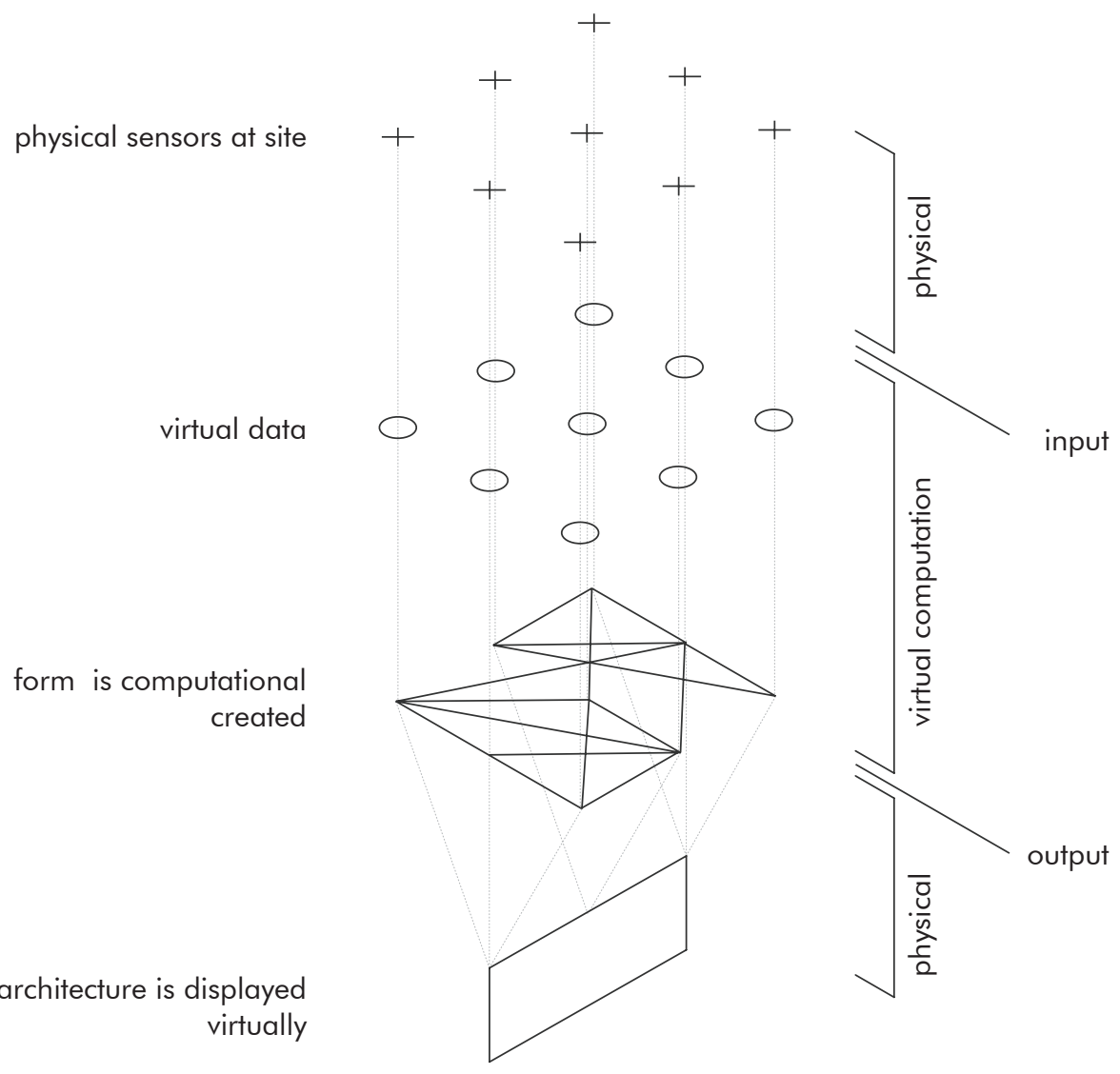

1.20 Conversion between physical site conditions to virtual reality architecture simulation. 

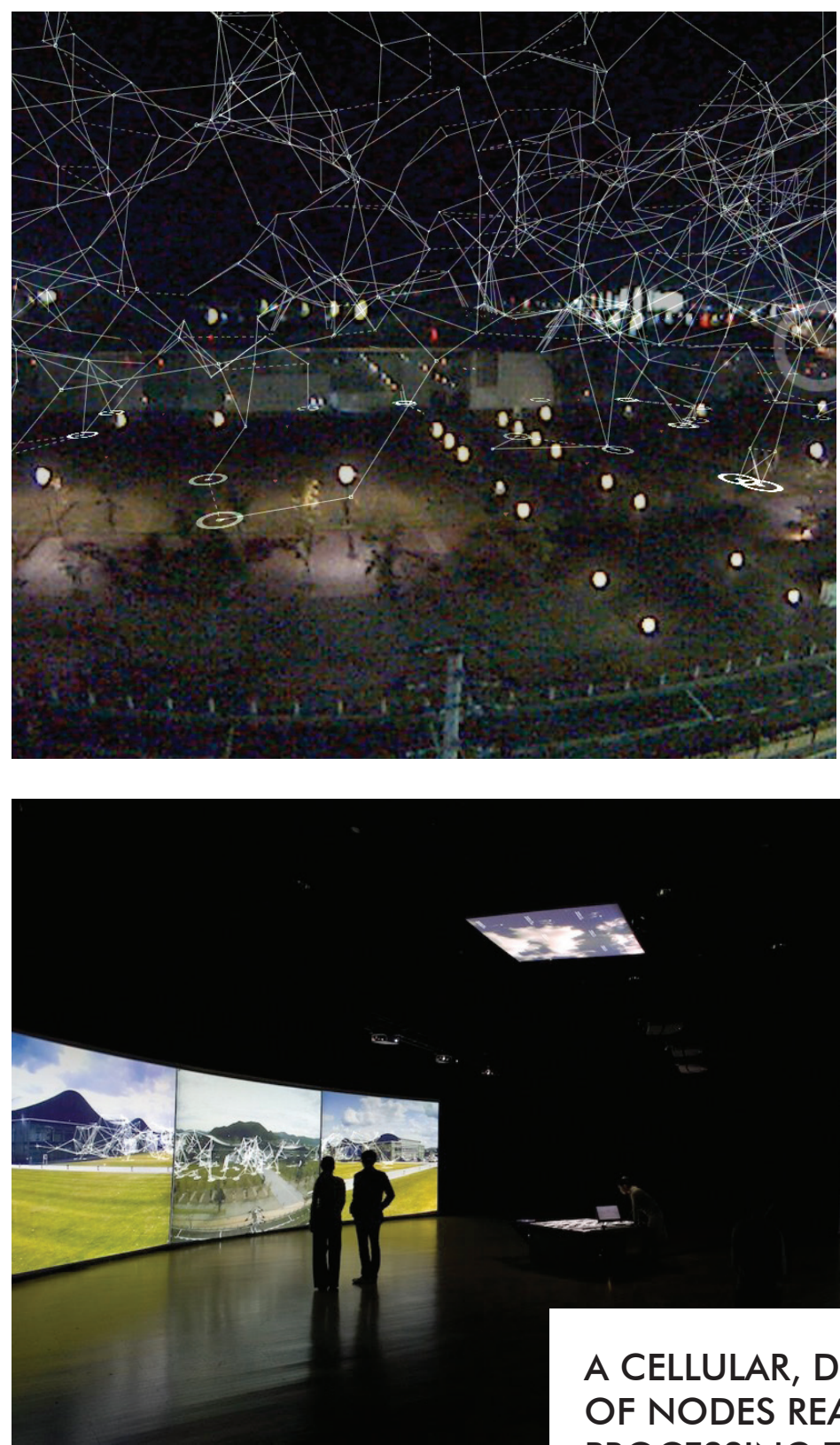

1.21 Digital images are augmented over a live feed of reality.

1.22 Output of responsive architecture transforming itself to site constraints and conditions.

-DOUBLENEGITAVES ARCHITECTURE 


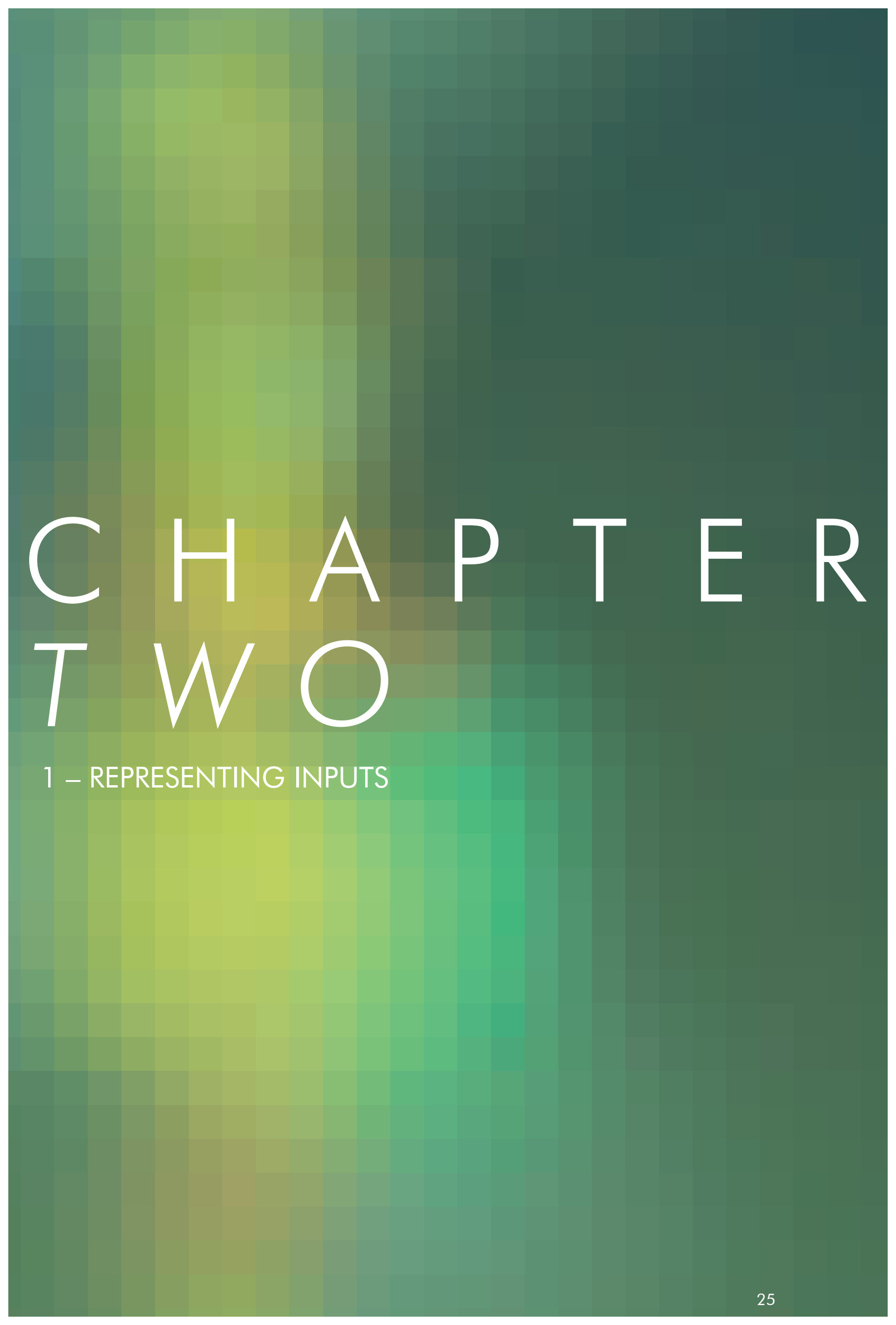


Translating data about people to information for people is done initially in two phases. The first represents data in informative $2 \mathrm{D}$ graphics through generic forms, the second spatialises the developed logic, creating a human-centric architecture.

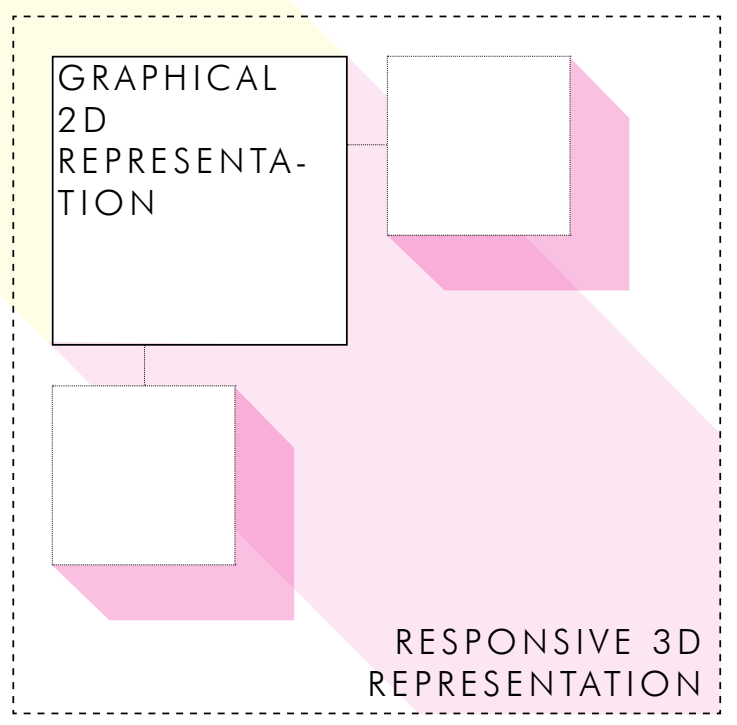




\subsubsection{WELLINGTON AIRPORT}

This international airport is situated in Rongotai, to the east of the Wellington CDB (fig. 2.02). The airport serves New Zealand towns and cities and Australian Cities, with 5.5 million passengers/year using the terminal (Infratil - Airports, 2015). With the first floor dedicated to departures, and the ground floor for arrivals, the airport lends itself to a testing ground for a multi flow way finding system (fig. 2.01). The medium size allows data from aircraft movements to be easily used to produce architectural interventions.

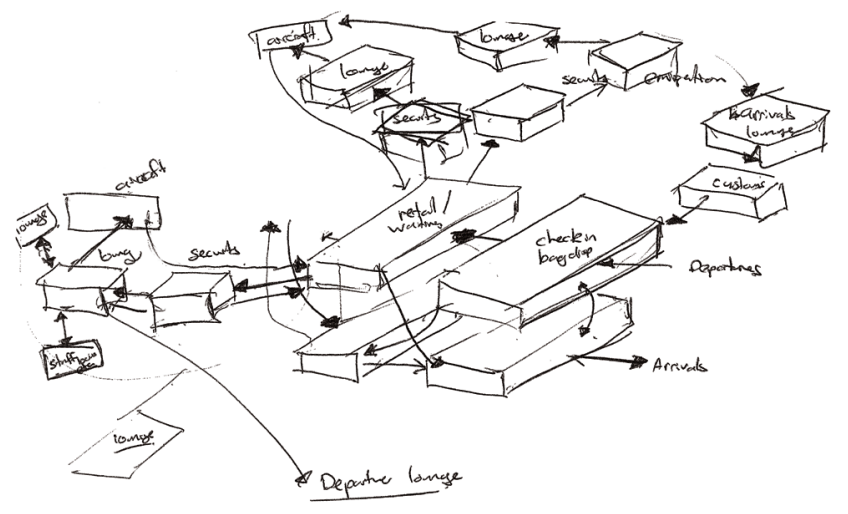

2.01 The organisation of Wellington Airport. 


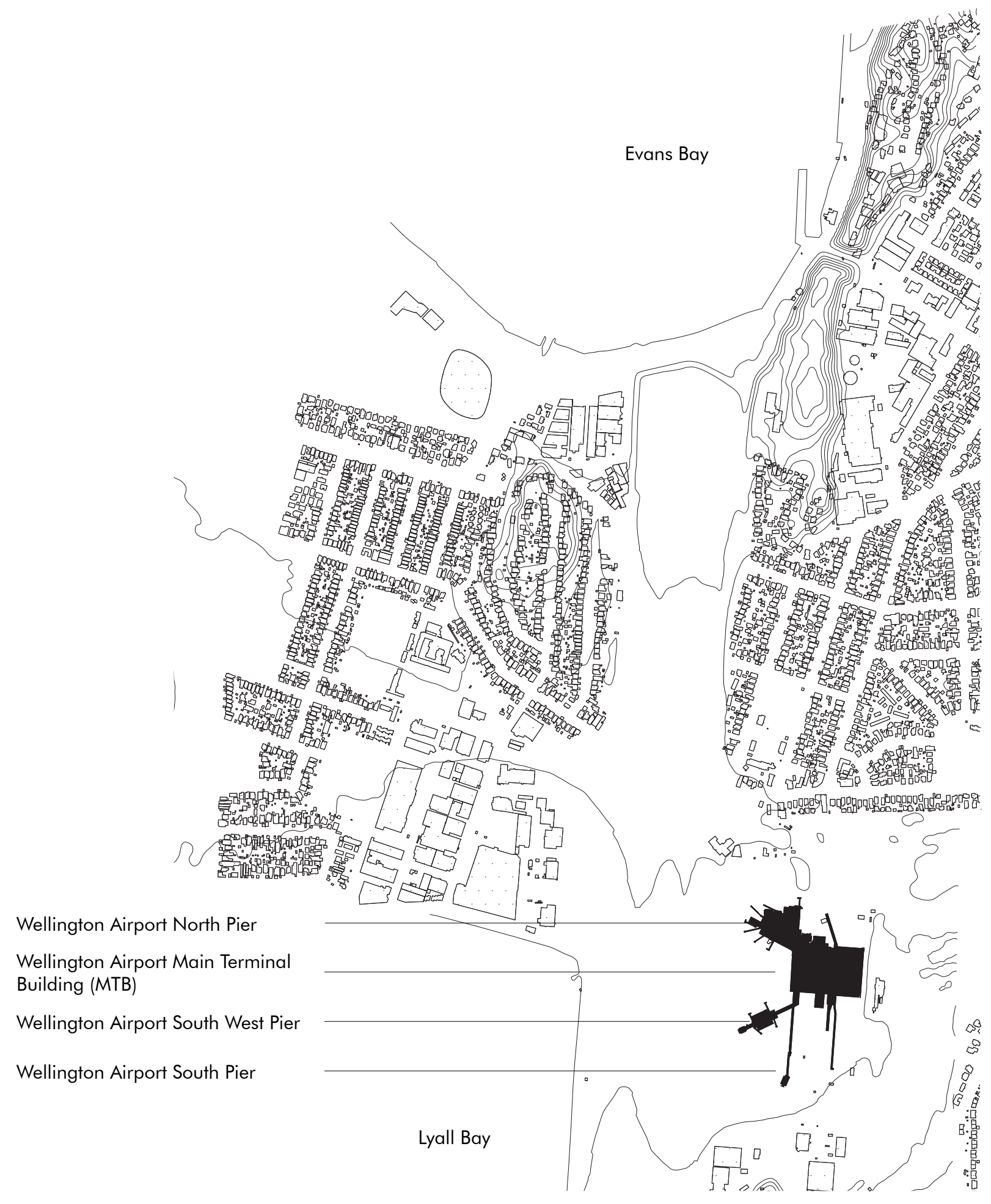

2.02 The location of Wellington Airport within Eastern Wellington, various parts of the airport are highlighted. 


\subsection{GRAPHICAL 2D REPRESENTATION}

Translation of live streaming data into graphic information is done using interactive visualisation design principles of data representation and graphic information design (Fry, 2008). Technology tests (Appendix B) are used as a starting point. Extracted information from personalised user centric data, including flight information, weather, geographical and human motion via motion capture, a depth of context and location is formed. Data choices are made on its centricity to users. A generic representation is designed around input data, encoding sequences to display multiple types of data inputs visually (Achten, 1997). Each data variable is mapped to a geometry's location, size or hue. Grasshopper completes this transformation, with specific plug ins to parse API and motion capture feeds.

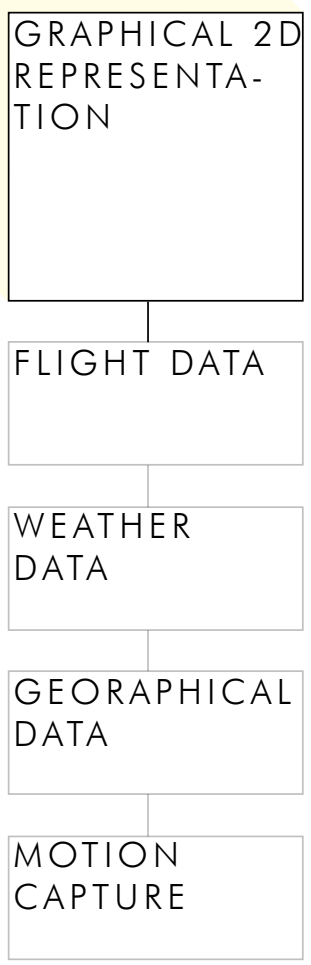




\subsubsection{FLIGHT DATA INPUT}

Real time arrival and departure data provides four outputs: time of flight, aircraft, gate and destination/origin. Data from online sources, using an API for an XML feed ${ }^{1}$, is mapped to show dynamic movement of users using geometry's size, location and movement as variables. The use of flight data instead of traditional, static way finding systems allows a parametric grasshopper script to receive data for any airport.

1 Flight Data is from flightstats.com XML premium data feed, allowing access to passenger airports globally.

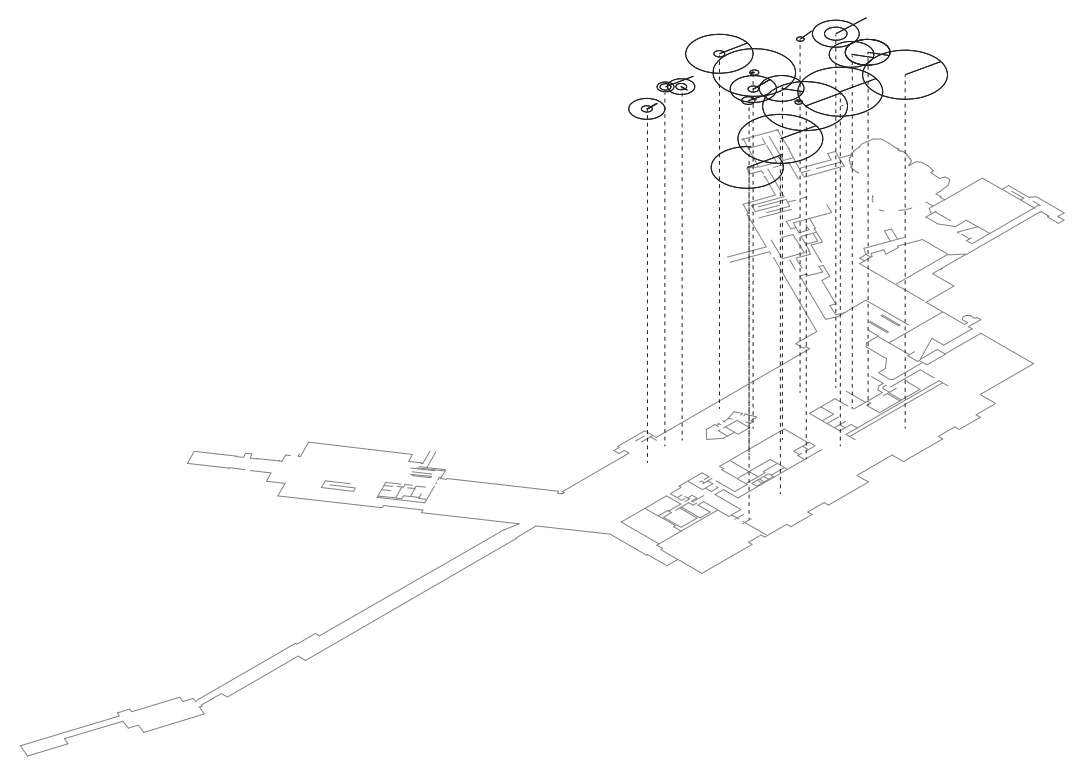

2.03 Mapping movement in the visualisation on the airport plan shows the indication of directionality based on departure piers. 


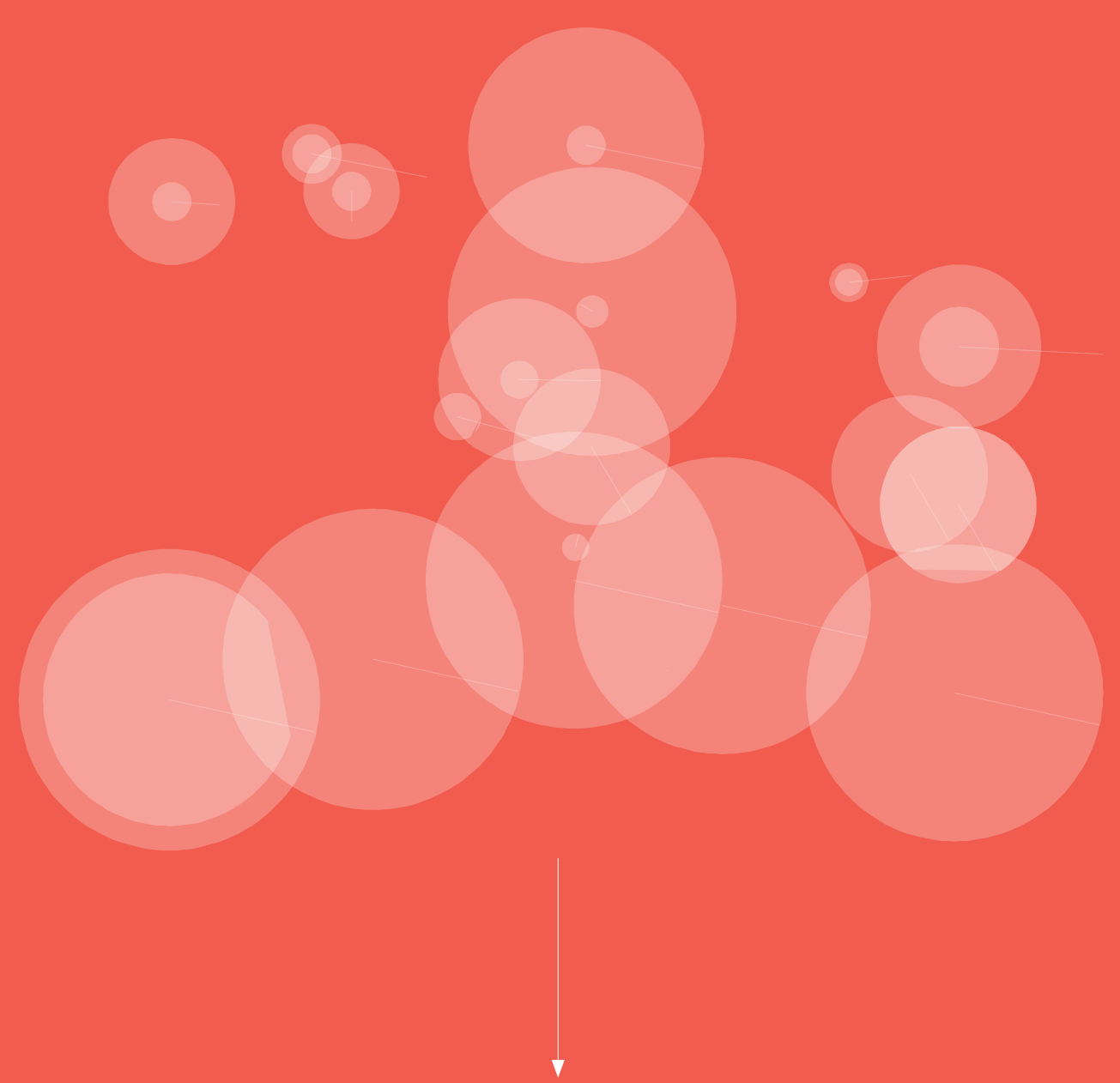

Each flight is represented as a circle

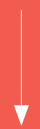

Radius is the amount of people on the flight

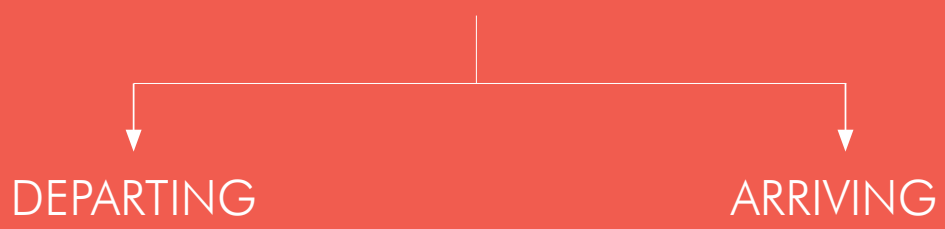

Each departing flight starts at the origin and moves further towards the gate as the flight gets closer to departure
Each arriving flight starts at the gate after the flight has arrived and moves further towards the origin 


\subsubsection{WEATHER INPUT}

Overlaid temperature or wind speed at the destination or origin adds contextual information. Colour coding explores a relative temperature level at the destination compared to the current location. This acclimatises users physically and mentally, allowing passengers to change their clothing or prepare for the destination through a simple graphic and a real time awareness of destinations' environment. This links locations to users based on their journey not physical distance.

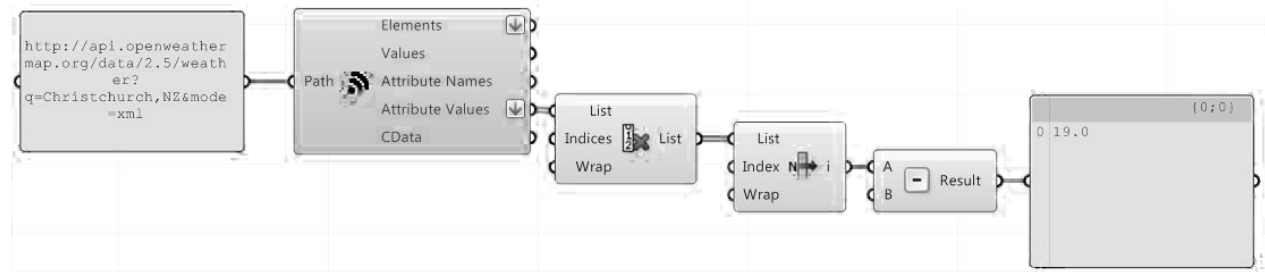

2.04 Grasshopper logic for the loading and parsing of real time weather XML files. The above shows the logic for Christchurch, NZ. This uses the gHowl plug-in. 

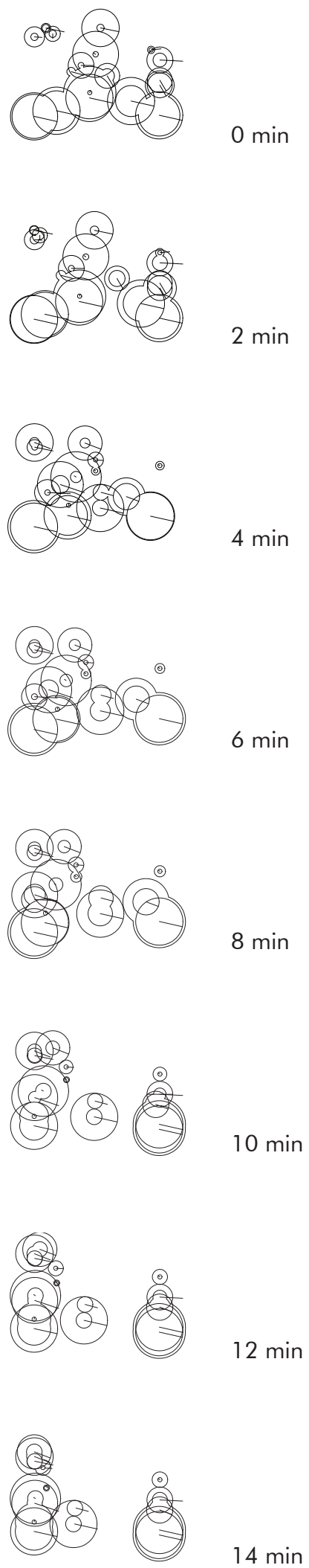

2.05 Movement of 2D representation at 2 min. intervals. 


\subsubsection{GEOGRAPHICAL INPUT}

Adding a geological contour map overlay of destinations' topography helps to understand the destination. Topological data is pulled based on the destination/ origin and becomes the surface, allowing the logic to become artistic, adding fluidity into a data driven system. 


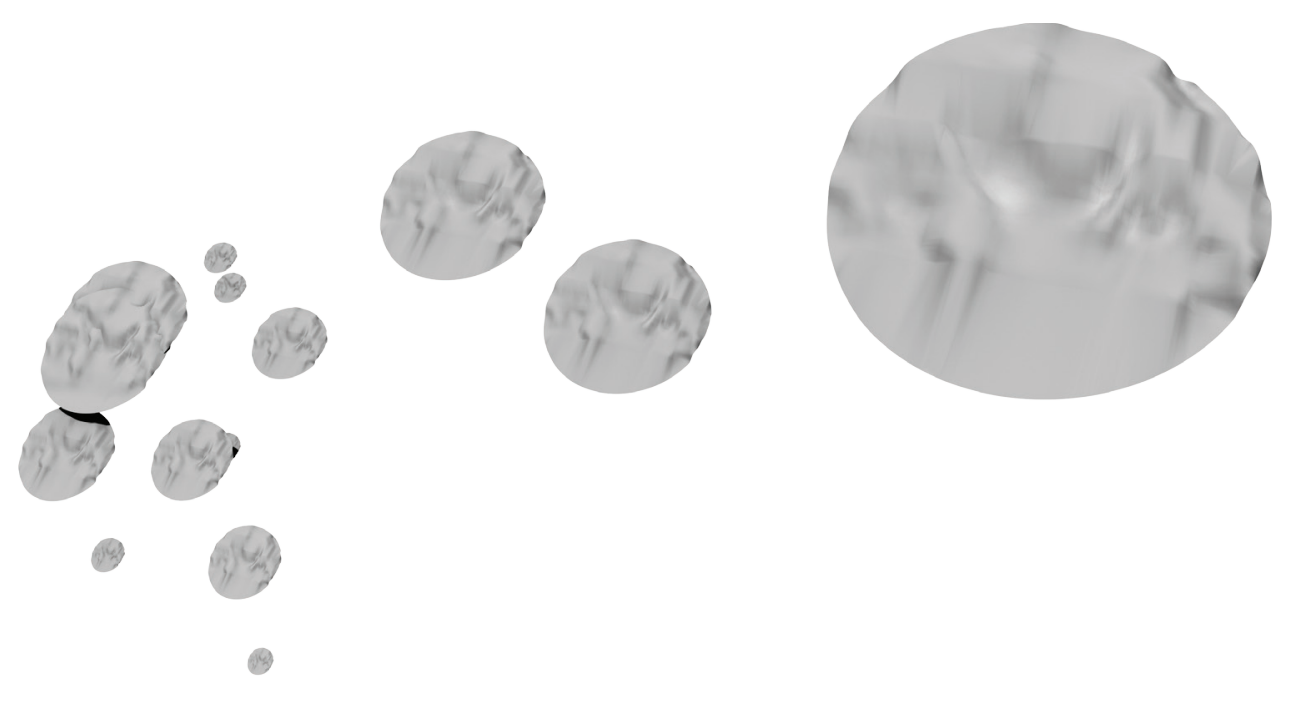

2.06 Geographic forms overlay the data points. A transformation up or down depending on temperature creates layers of forms. The visual language and knowledge of locations landscapes creates a simple communication method. 


\section{1 .4 KINECT INPUT}

An interaction method was devised where the location of a person is motion captured $^{2}$ and mapped to the $2 \mathrm{D}$ visualisation, acting as a negative attractor to shift data around. This introduces a layer of embodied interaction to virtual forms. The human point is mapped to various parts of the body, and could be extended with a different interaction method with the user's own data (i.e. their flight).

2 "Motion capture is the process of recording a live motion event and translating it into actionable data that allows for a $3 \mathrm{~d}$ recreation of the performance" ("Motion Capture Software and Mocap Tracking Info,” 2015) . 

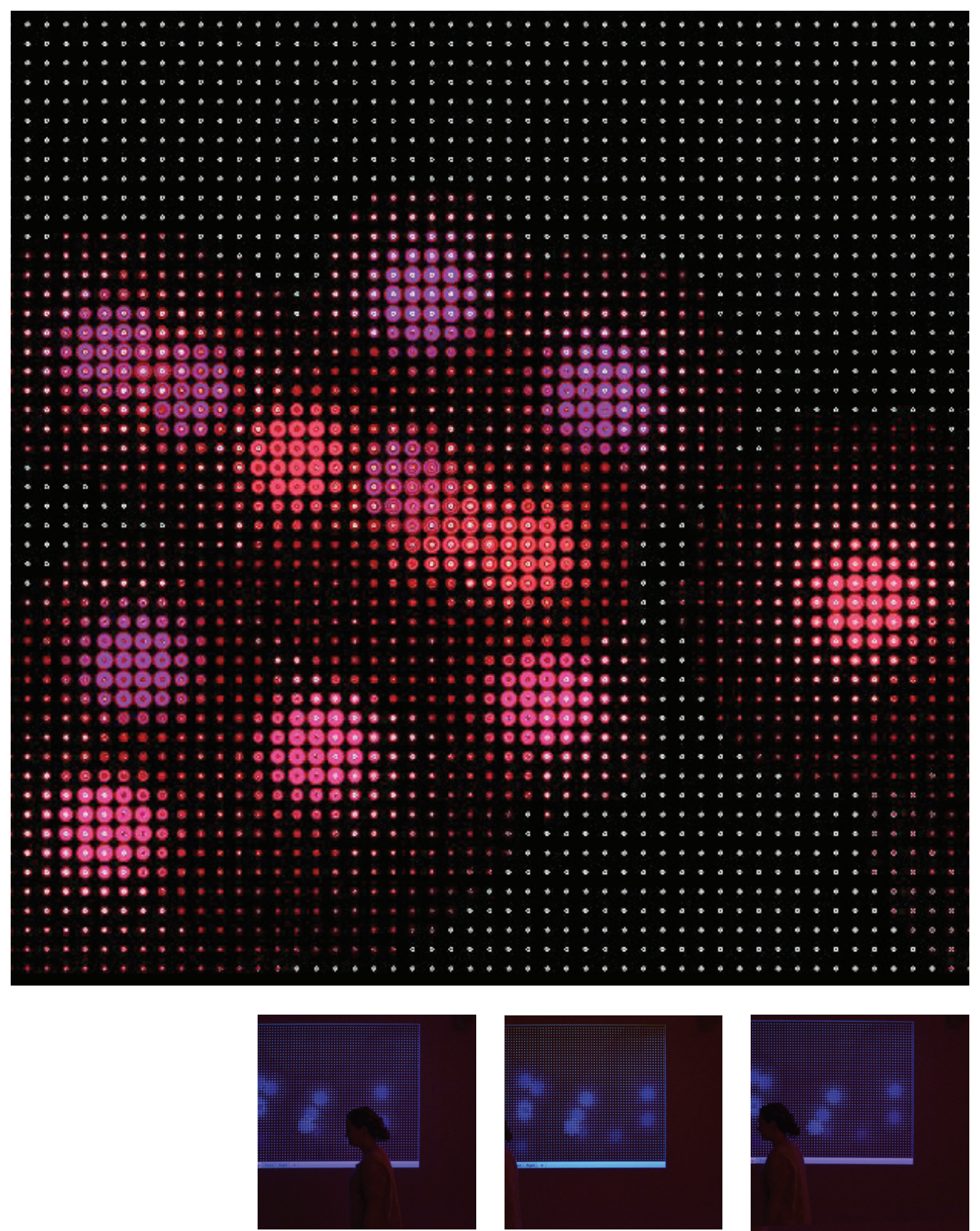

2.07 Projection onto a screen showing flights and weather data. 2.08 Photographic series of interactive data projection with motion capture input. The physical scale to digital data will inform the next steps of the project. 


\section{1 .5 SUMMARY}

These initial experiments provided a base knowledge for data integration into a system, transforming into a visually understandable medium. A base knowledge of the input of data sources is beneficial later in the project, providing new ways of displaying this. Decisions on which data to highlight is from the closeness to people. Mapping this makes data more useful in context, resulting in surfaces (fig. 2.09), which have communication through an embodied atmospheric interaction, although these need to be spatialised.

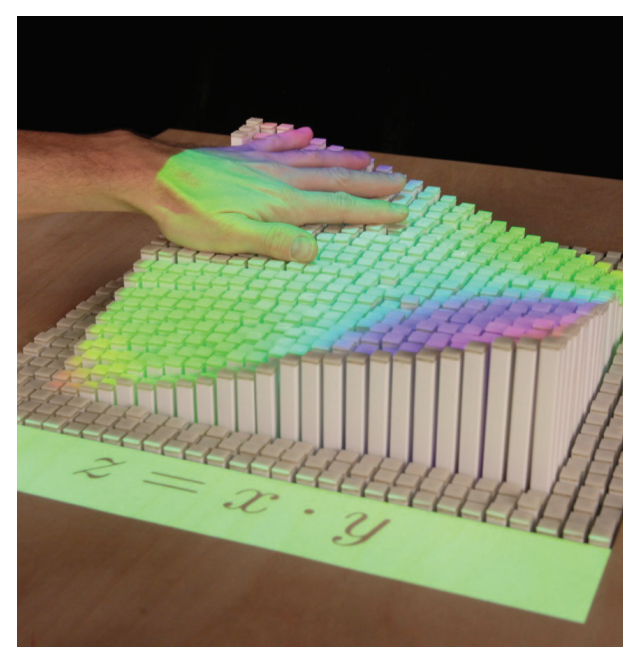

2.09 MIT Media Lab's Tangible Media Group's inFORM is a Dynamic Shape Display which creates digital physical surfaces to move objects and 'interacts with digital objects in a tangible way'. This could be used to move users around the space through kinetic form - the creation of a physical data landscape. 


\subsection{RESPONSIVE 3D REPRESENTATION}

Using the previous $2 \mathrm{D}$ methods, this phase creates a kinetic space responding to people via input of data. Wu and Clayton (2015) define a 3D visualisation of data sources which maps variables in $3 \mathrm{D}$ to create objects. Data is defined in $3 \mathrm{D}$ by the angles, size, porosity and location of circular shapes, which are then lofted together to create distinguishable forms based on the data set. Using similar methods, in a kinetic form, responsive architecture is created, using a series of steps to develop a central concourse form. These are:

- Gate links flight to pathway which are designer defined.

- Time until flight arrives/departs calculates a point on a path.

- Points creates a curve using 2D metaballs.

- Vertical extrusions inside the curve remain flat, allowing more space for users.

- Vertical extrusions outside the curve are extruded by an amount proportional to the distance to the curve.

The result is an extending and retracting form, creating enclosed or exposed space, seating or walls which cause circulation, acoustic, light and material properties to change and help people navigate spatial zones (fig. 2.10).

The developed space takes cues from Philip Beesley's Hyzolic Ground, an analogue output of digital computation. The purely physical space involves large amount of computing, determining physical kinetic movements. However, this case study pulls its data directly from the environment, by using online but contextual data, the thesis project remains site specific.

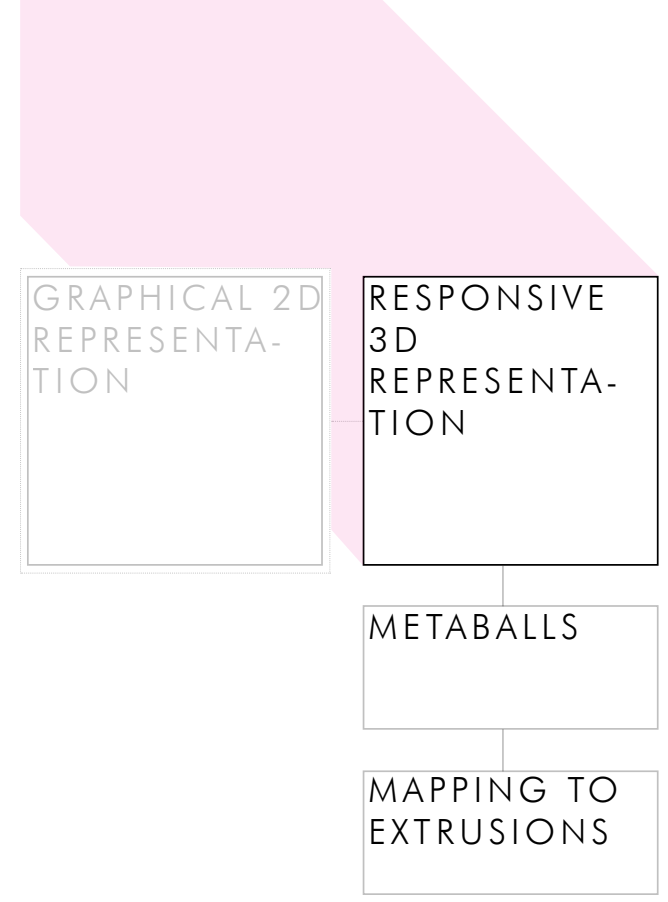



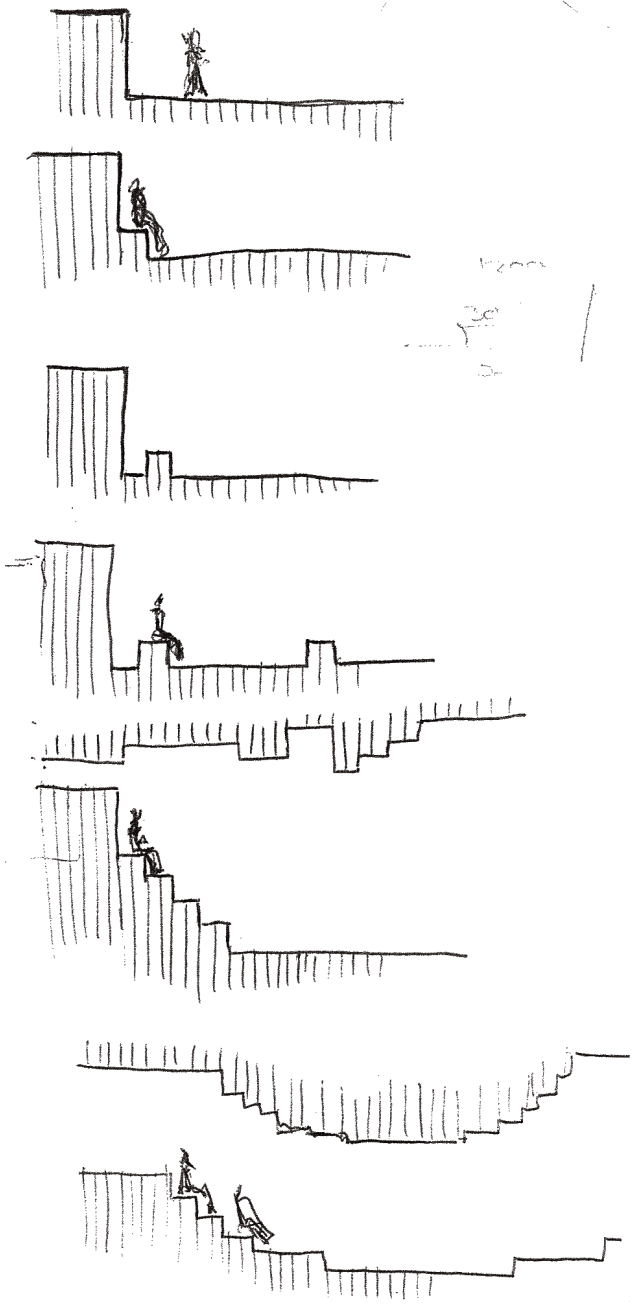

2.10 Types of arrangements of space, influenced through movement of space based on data. 


\subsubsection{D LOGIC}

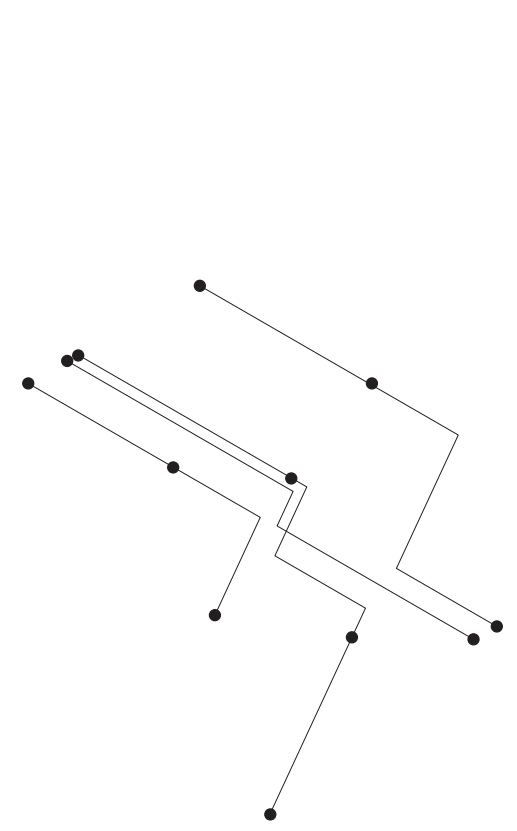

\section{PATHWAY}

Routes between concourse and gates for arriving and departing travelers.

2.11 Pathway routes through airport concourse, predefined by author.

\section{POINTS}

Each flight moves along path towards or away from gate.

\section{TIME}

As time changes the flight moves closer/away from gate.

\section{SIZE}

The radius of the point is set by the number of passengers \& grows as time increases. 
PATHWAYS

Flights have a point on the pathways lines.

P METABALL

A 2D metaball form is created for each pathway informed by the location and strength of the flight points.

2 SURFACE

A surface is formed based on their proximity to the 2D metaball forms.

\section{GEOMETRY}

The height of the surface at the centre of the extrusions sets the height for the floor. The ceiling is inversed.
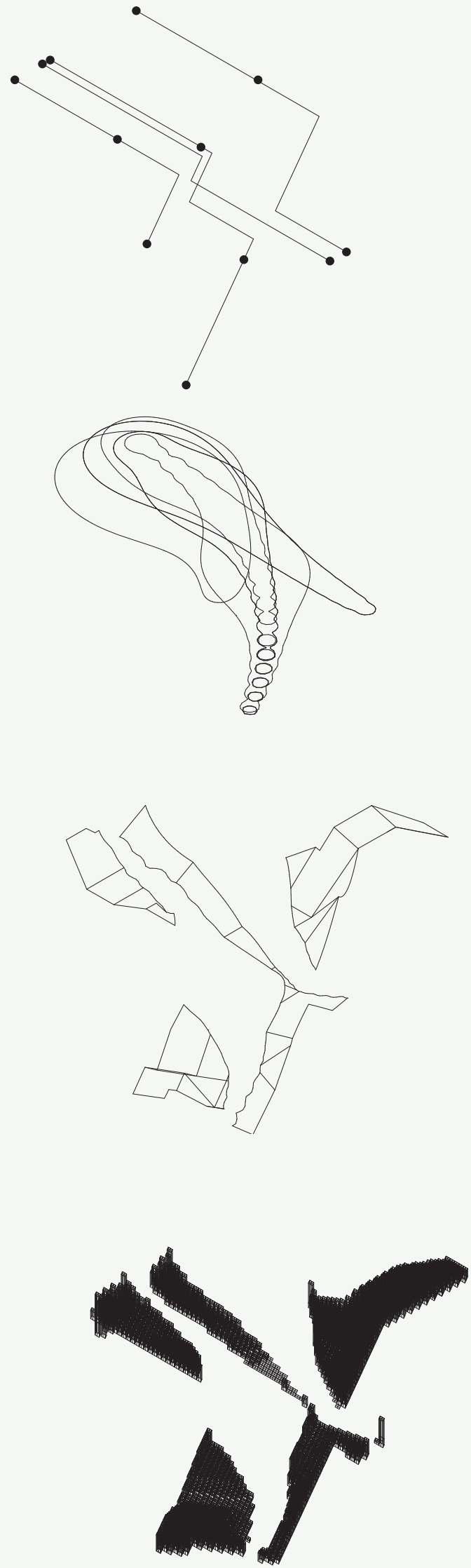


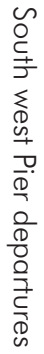
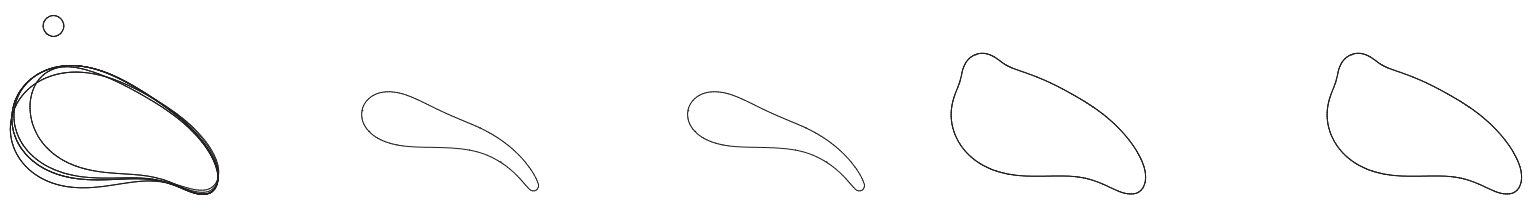

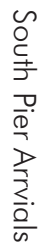
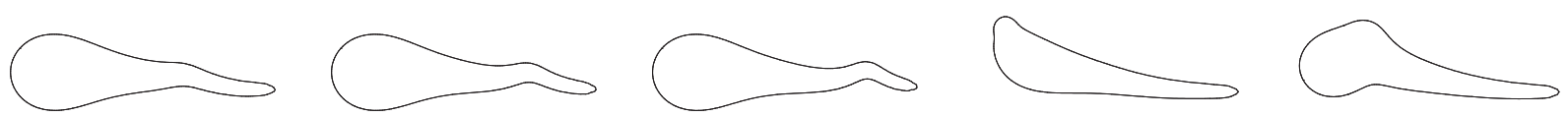

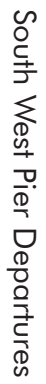

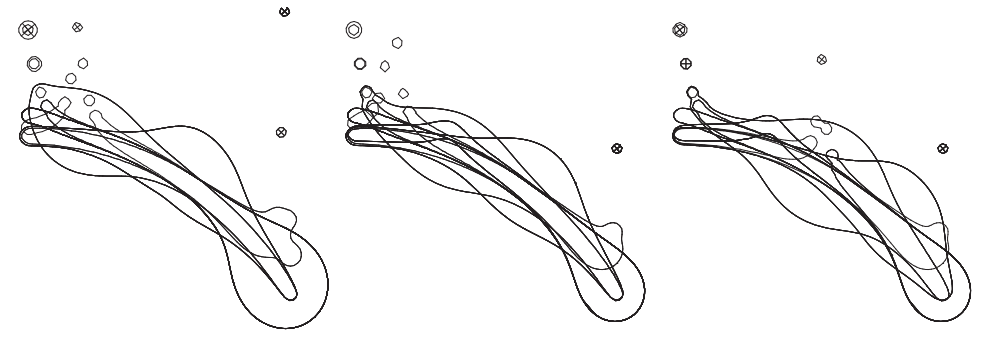

0
0
$C$
5
5
70
0
0
0
0
0
0
$\frac{0}{c}$
$\frac{1}{0}$
0

\begin{tabular}{l|l}
46 & $\begin{array}{l}\text { Representing } \\
\text { Inputs }\end{array}$
\end{tabular} 

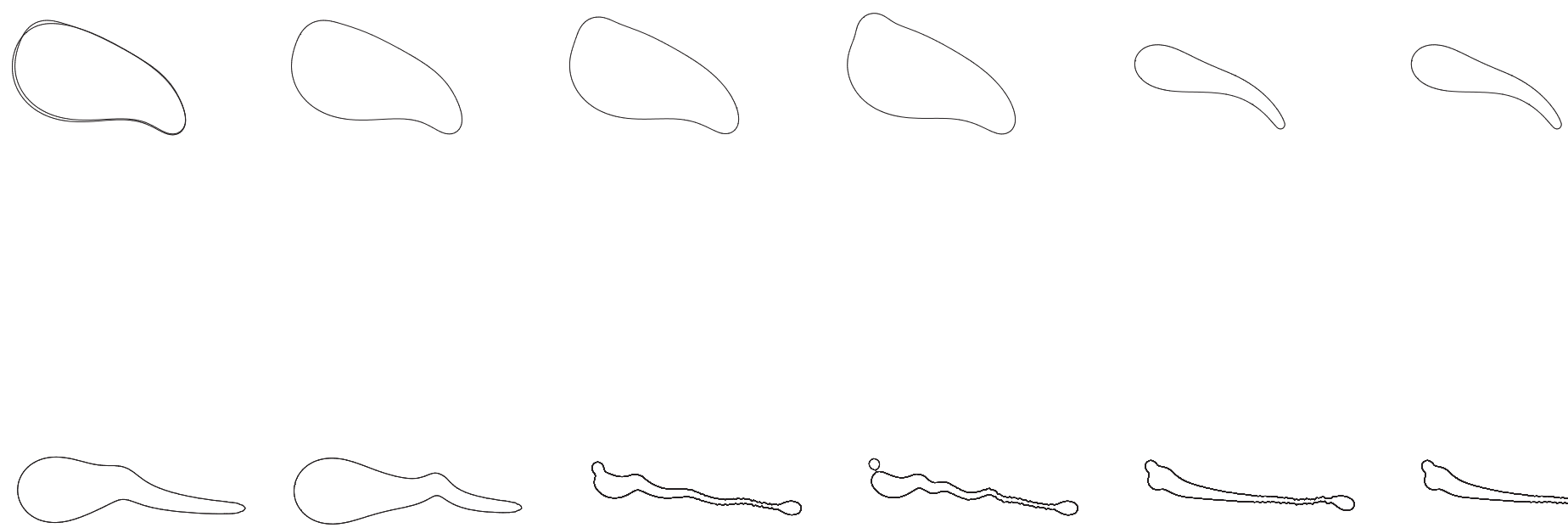

$\sqrt{2}$

$\overbrace{}^{2}$

Q

$\sqrt{2}$
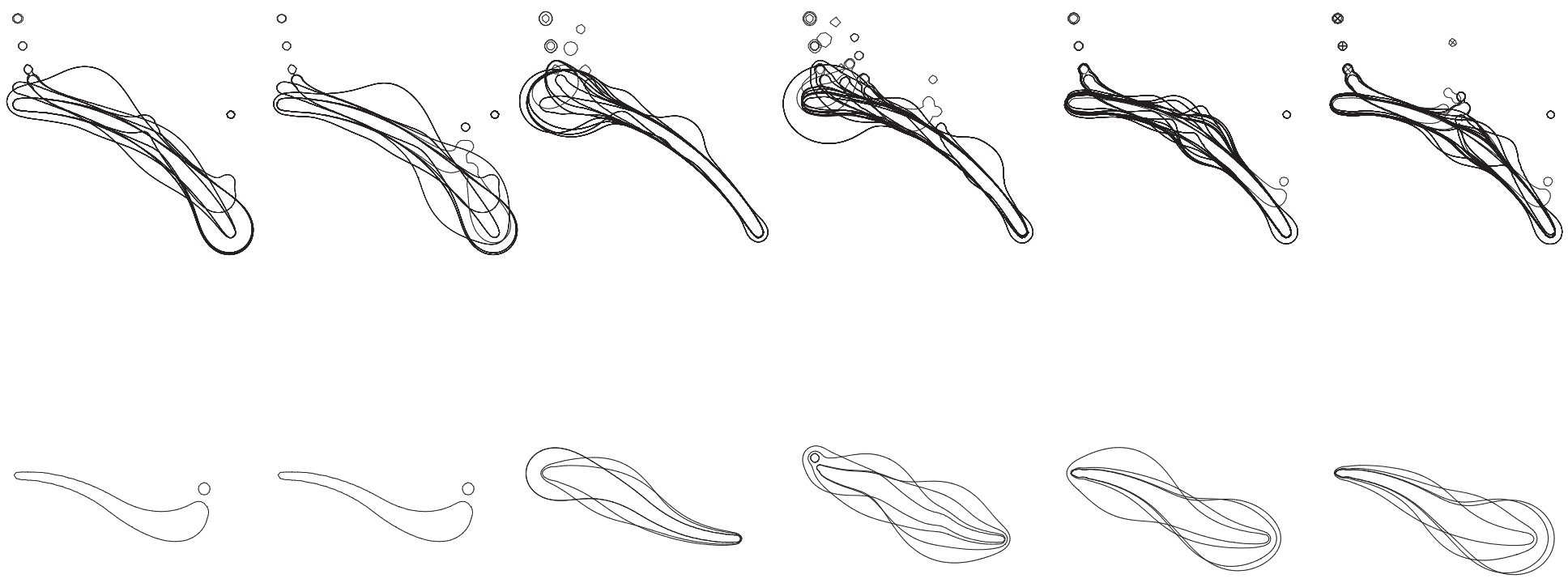

47 


\subsubsection{DRAWINGS}

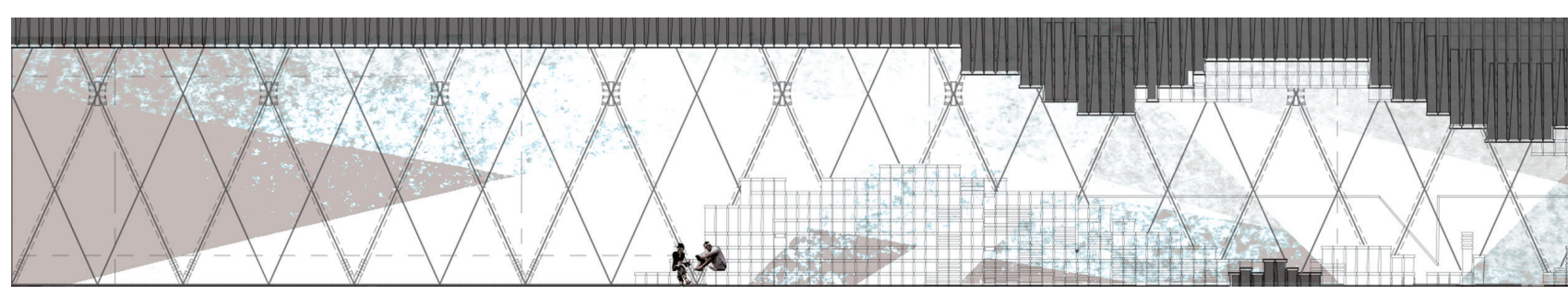

Section A

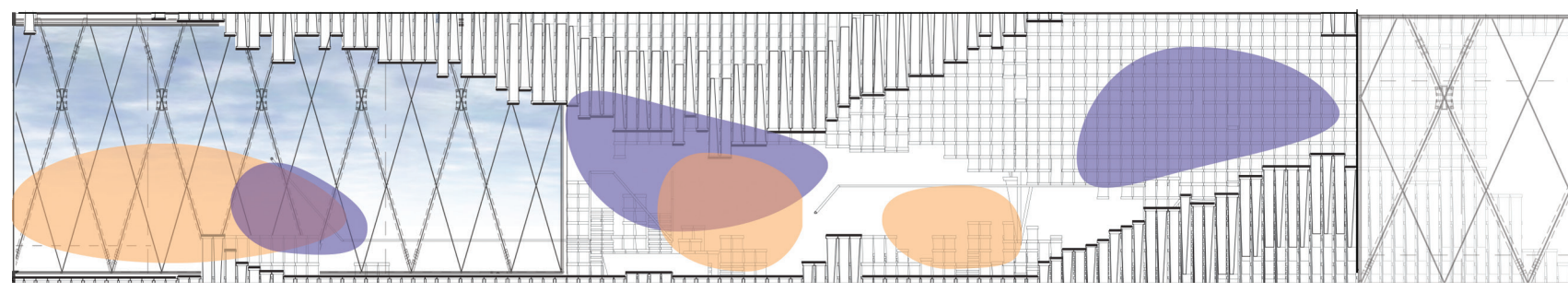

Section C

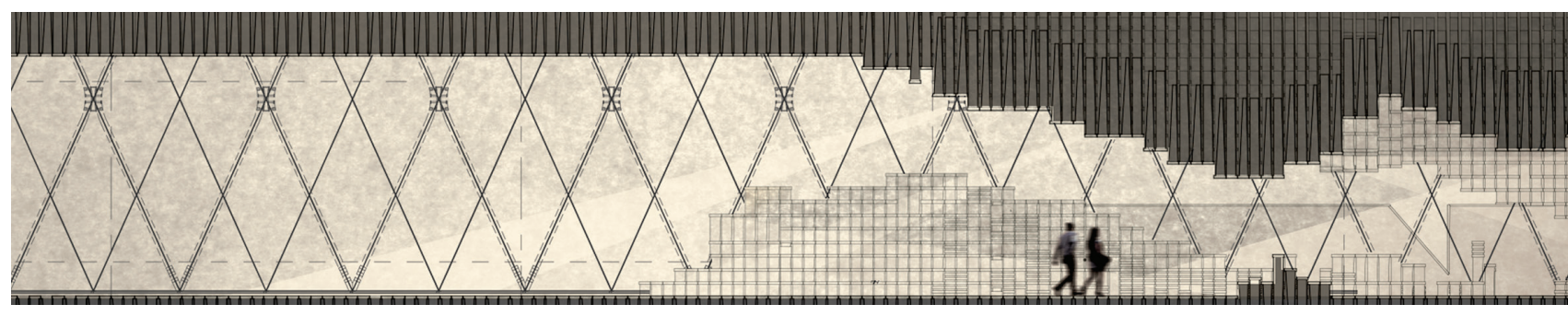

Section B
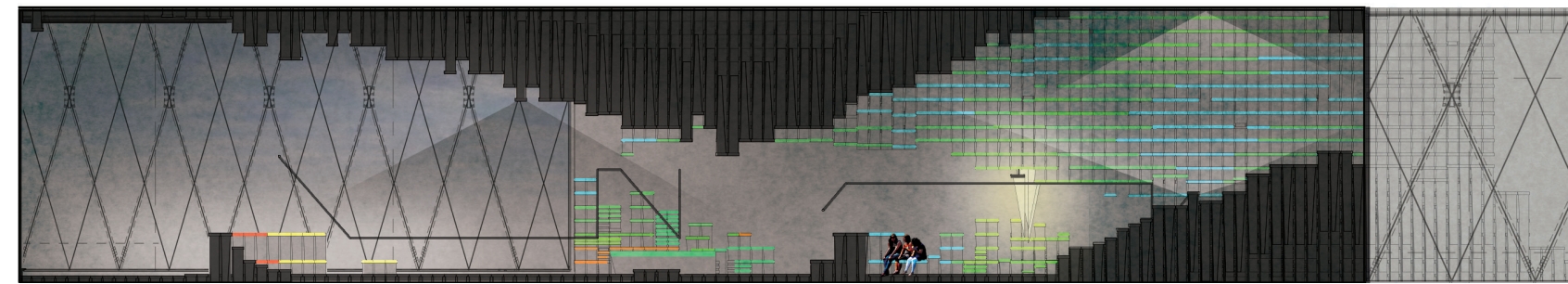

Section D 


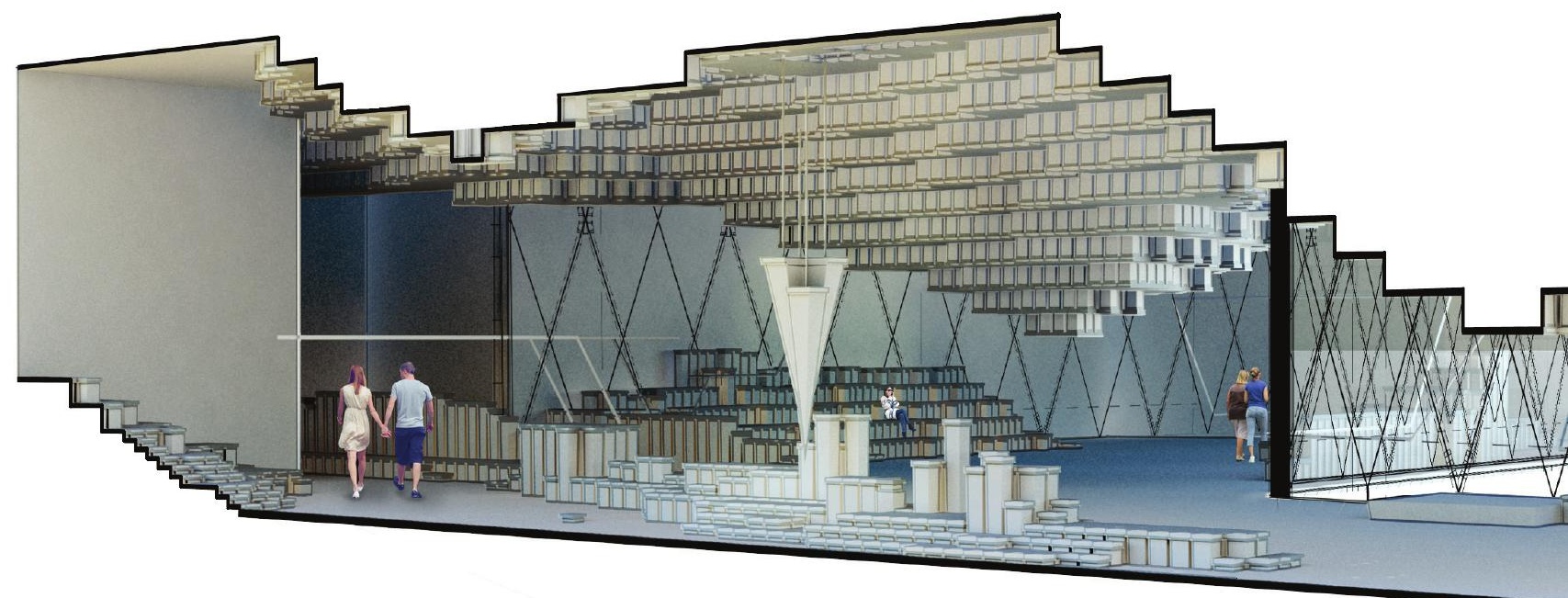

2.17 Perspective section of the airport installation with pathways carved out of the surface, the edges become seating. 

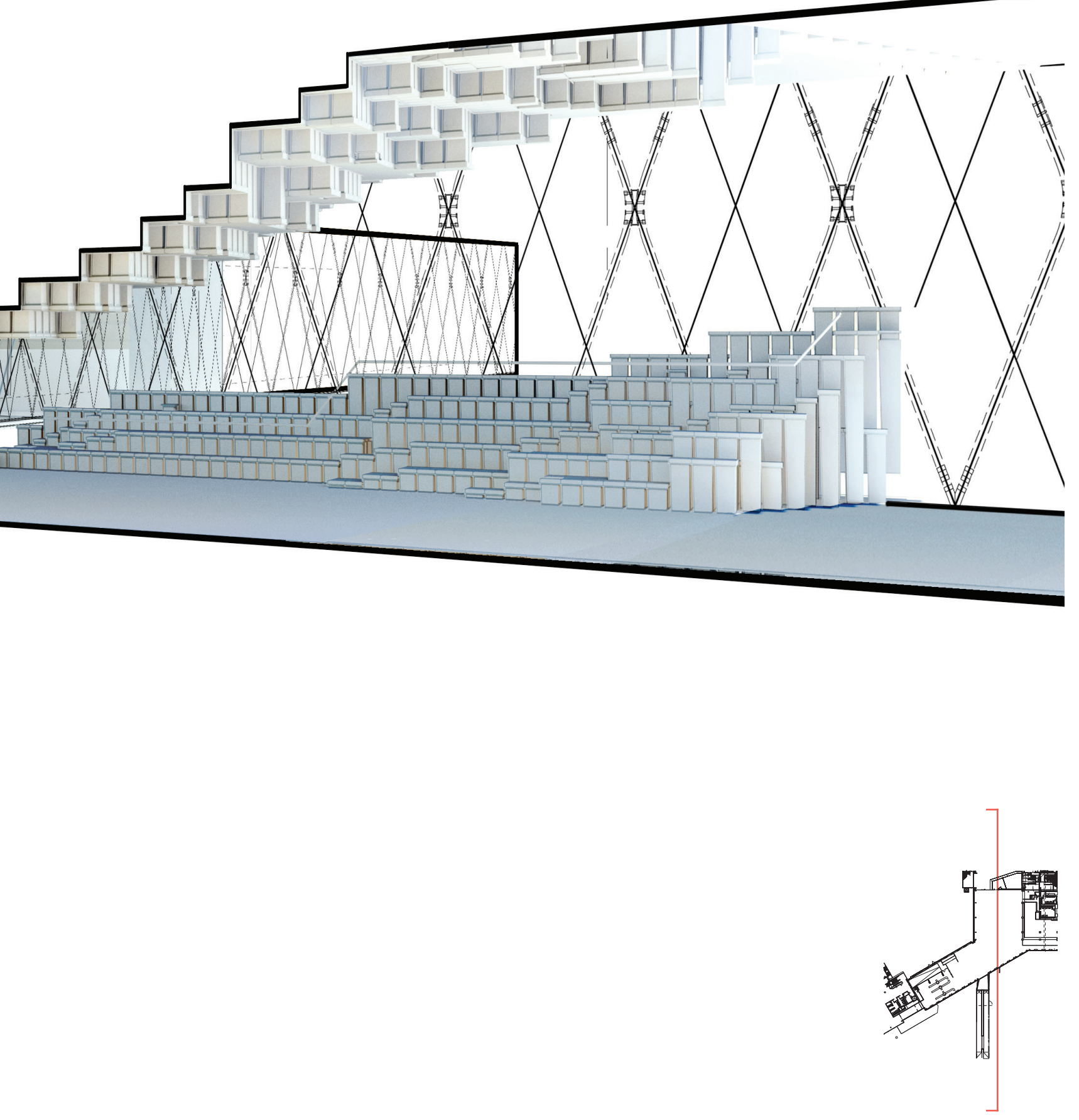


\subsubsection{RESPONSIVE ARCHITECTURE FROM DATA ABOUT USERS}

The architectural form responds to users' needs across the day, informed by flows of people through space, represented in data. Big data, indirectly from user's movements, allows the architecture to become a compound of user's paths, responding to trends with awareness for future events, 'corresponding to certain time frames' (Hansen, 2015). The process provides an extension of social groupings and spatial flows beyond pre-existing norms.

This initial project meets some of the performance index, however the interaction method is fully physical so is in actual reality. There is a strong link between users and space, and the virtual data linking to physical form. It lacks the extension of spatial and social contexts, as explained below.

Source data inputs effect the spatial outputs. As there is no data distinction between visitors and residents, the design cannot respond to these changes. Such inputs would change performance when more visitors were exiting or entering the city compared to residents, creating more complex logic but a refined form. Real-time changes in data allow proportions of seating, social and circulation areas to alter in real time. Responsiveness creates social areas, but as personal data isn't used as an input, social interaction is not highlighted. Colour coding funnels users based on flows and changing lighting in key pathways gives space a transformative directionality to it. 


\subsubsection{SUMMARY}

The bidirectional navigation for a single airport space embeds parameters which change based on the current data, accurately suiting the needs of the users. A language for understanding data in physically inhabitable, three dimensional ways is developed. 


\subsection{SUMMARY OF PRELIMINARY DESIGN STAGES}

The stage highlights difficulties of translating real-time data at an appropriate level of detail while remaining responsive to all users' needs. It was successful in developing methods of displaying data visually, showing that data visualisation contains important precedents. The need for kinetic architecture is questioned; the actual movement could become a gimmick and unrealistic. Information can be relayed through material, light and sound. Interaction between people and space is aimed to be improved by including more real-time human inputs and a composite system between traditional way finding mechanisms and realtime personalisation available with technology.

The qualitative assessment on the Mixed Reality Design Index (fig. 2.18) provided insights for the next project, a focus of readable real time personalised information transmission is needed. Many way finding techniques have not been included, instead focusing on interpretation of data inputs into simple and ergonomic forms. 


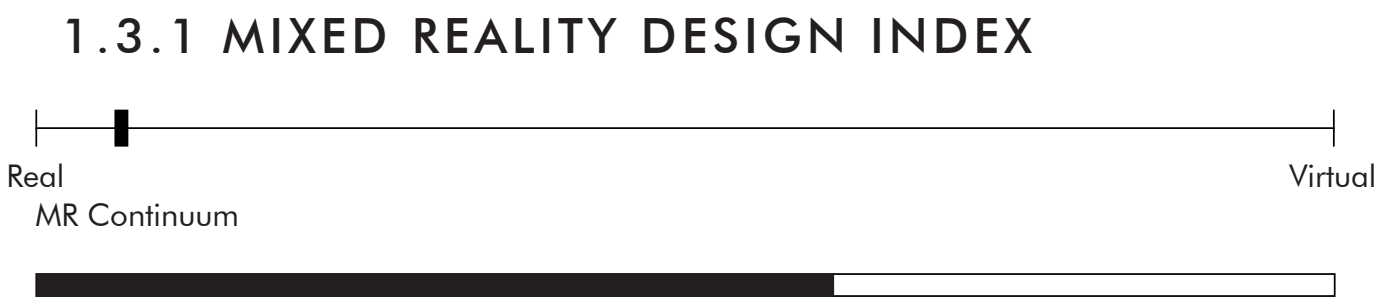

Comprehensive space between material, cognitive and symbolic

Corroboration and reciprocity between physical and virtual components

Extension of a coherent spatial experience and social context.

Anthropic Design

2.18 MR Design Index for first design stage, highlighting possible areas of improvement for the next design stage. The level of personalisation is to be increased, along with the way in which the architecture can create social space. 


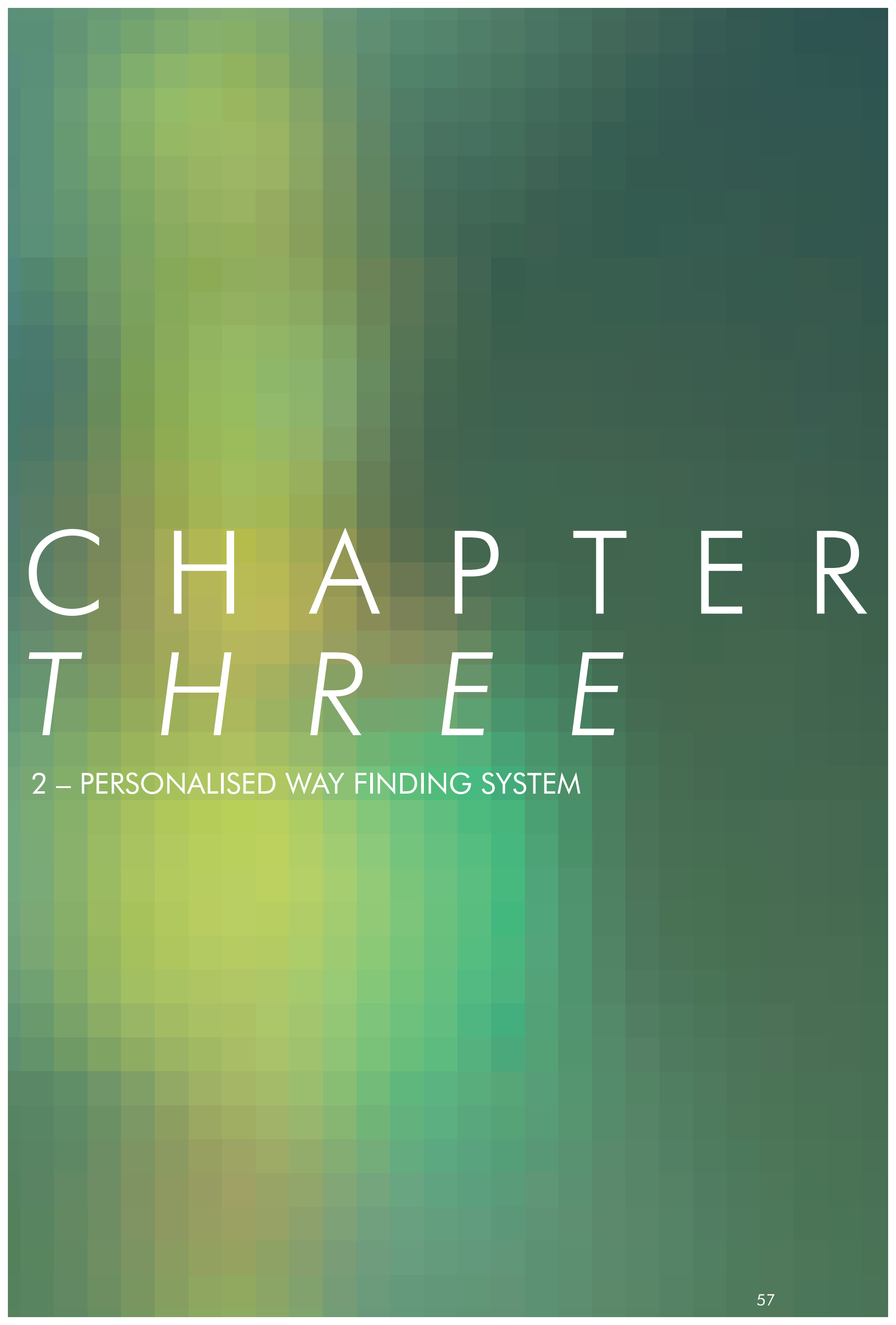


Based on a design review, the project shifts focus to allowing pervasive technologies to create spatial technology systems centred on personalised social interaction and way finding. As humans are central to the project, a sense of individuality must remain throughout the experience of shared space. The initial section translates data into a real time $2 \mathrm{D}$ representation of space, then uses computational overlays to induce further human understanding. The second phase uses similar techniques to take static data to create forms as the physical element of the spatial system. Finally, a personal Human Computer Interaction (HCI) method is developed to allow a fuller level of embodied interaction with the designed system and the implementation of location models into virtual space.

Unnumbered Sparks and Points influence this new system. Conceptually, the design started as an interior kinetic façade (Moloney, 2011). Unnumbered Sparks' system of displaying information, users touching phone screens via projections, on a surface creates a spatial experience from common technology. This is used as a model for the project. A Mixed Reality machine, with users having a physical sensorial connection with sight and sound, works well in the large public space. Smartphones are essentially controllers, acting as inputs to the system. This is similar to the current project, with form and projection being linked to create a continuous form that is accessed in a public space.

Points' use of real time data to alter the experience will also be used. Transforming data inputs based on its location and users, creating a context aware system. This external input, used with flight arrival and departure data, will make for user flow driven experiences.

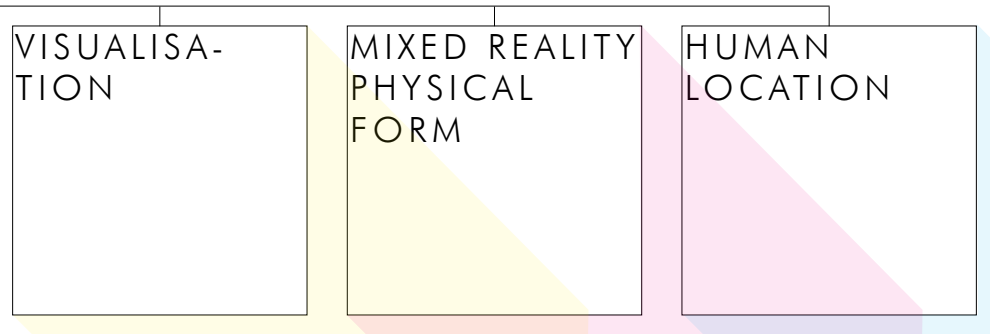




\subsection{VISUALISATIONS}

\subsubsection{BASE PROJECTION}

Processing ("Processing.org," n.d.), an Integrated Development Environment and language often used for interactive graphics, is used to develop a simulation of space as a Cartesian coordinate system. This is used instead of Grasshopper and Rhino because of its performance with large amounts of data and real time processing. Design iterations developed a logic for the detailed display of data. The initial logic produces ever changing forms depending on data. All screenshots of the visualisation work off live data, and move in real time.

\begin{tabular}{|c|c|}
\hline \multicolumn{2}{|c|}{ VISUALISATION } \\
\hline FLIGHT DATA & ABSTRACTION \\
\hline $\begin{array}{l}\text { USER } \\
\text { SELECTION }\end{array}$ & $\begin{array}{l}\text { SPECIFIC } \\
\text { ARCH VISUALI- } \\
\text { SATION }\end{array}$ \\
\hline FLOCKING & $\begin{array}{l}\text { MOTION } \\
\text { CAPTURE }\end{array}$ \\
\hline
\end{tabular}




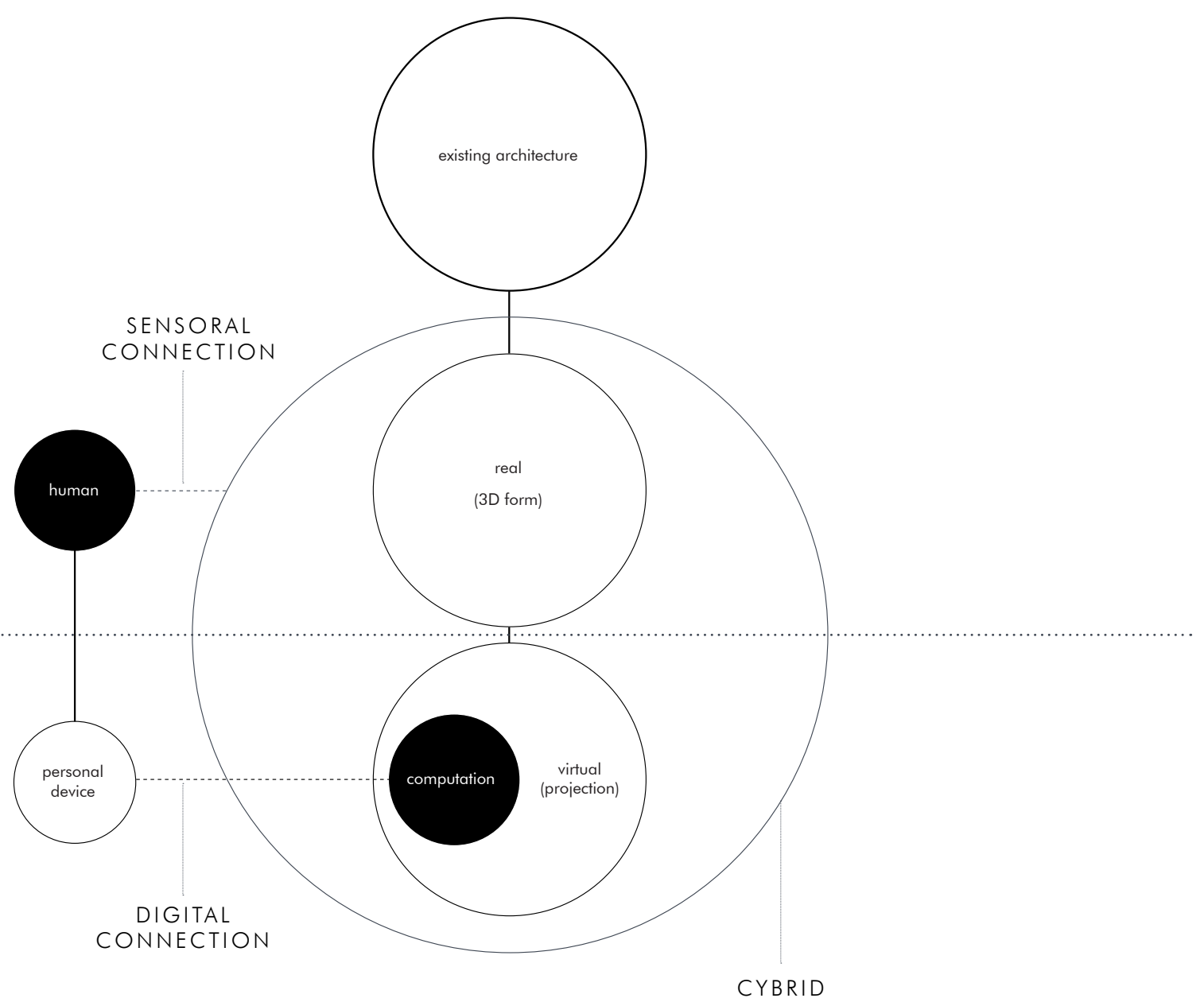

3.01 Composition of the proposed cybrid and its connection to humans, with methods of interaction. 


\section{DESIGN REVELATION \\ - USER INTERFACE METHODS FOR VISUALISATIONS -}

Between the way a system works (implementation model) and the way a user understands and believes it works (mental model), there are represented models. The further towards the mental model the interface and interaction is, the more user friendly it is (Cooper, 2007). As users inherently understand spatial principles, inserting a virtual simulation of space as a represented model will help users understand the information behind the data in more detail (fig. 3.02), the creation of an 'information environment' (Cooper, 2007). Methods include the software being considerate and non-intrusive, and having actions and goal driven interactions. Visual designing with variables such as position, texture, orientation, hue, size and shape are key.

\section{"THE SOFTWARE SHOULD ACT LIKE A CONSIDERABLE HUMAN BEING".}

- COOPER

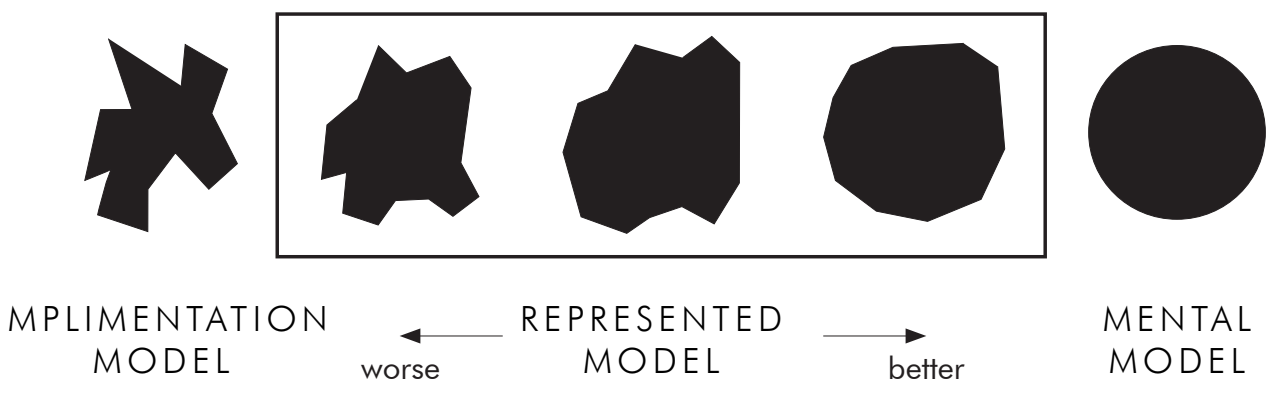

4 3.02 Implementation model, represented model and mental model from User Interface design guidelines. This has an application in creating design that is as close to the mental model, how users perceive space, while being technically possible.

$\rightarrow 3.03$ A segment of XML flight data input for two hours on July 162015 . The complexity of it causes issues. The data is available for any public airport and displays many variables including those seen on airport flight status screens. 
- 〈flightStatuses>

- 〈flightstatus>

〈flightId >571469611</flightId >

<carrierFsCode $>\mathrm{VA}</$ carrierFsCode>

〈flightNumber >104</flightNumber>

<departureAirportFsCode>BNE</departureAirportFsCode>

<arrivalAirportFsCode $>$ WLG</arrivalAirportFsCode>

- <departureDate>

〈dateLocal>2015-07-16T08:50:00.000</dateLocal〉

$\langle$ dateUtc>2015-07-15T22:50:00.000Z</dateUtc〉

$</$ departureDate>

- <arrivalDate>

〈dateLocal>2015-07-16T14:15:00.000</dateLocal〉

$\langle$ dateUtc>2015-07-16T02:15:00.000Z</dateUtc>

$</$ arrivalDate $>$

$\langle$ status $>$ L</status >

- 〈schedule>

<flightType> J </flightType>

〈serviceClasses>JY</serviceClasses>

$\langle$ restrictions />

$\langle$ /schedule $>$

- 〈operationalTimes>

- <publishedDeparture>

〈dateLocal>2015-07-16T08:50:00.000</dateLocal〉 $\langle$ dateUtc>2015-07-15T22:50:00.000Z</dateUtc>

$</$ publishedDeparture>

- 〈publishedArrival>

〈dateLocal>2015-07-16T14:15:00.000</dateLocal〉 〈dateUtc>2015-07-16T02:15:00.000Z〈/dateUtc〉

$\langle/$ publishedArrival>

- 〈scheduledGateDeparture>

〈dateLocal>2015-07-16T08:50:00.000</dateLocal> $\langle$ dateUtc>2015-07-15T22:50:00.000Z</dateUtc〉

$</$ scheduledGateDeparture $>$

- <estimatedGateDeparture>

〈dateLocal>2015-07-16T09:19:00.000</dateLocal〉

$\langle$ dateUtc>2015-07-15T23:19:00.000Z</dateUtc〉

</estimatedGateDeparture>

- 〈actualGateDeparture>

〈dateLocal>2015-07-16T09:19:00.000</dateLocal>

$\langle$ dateUtc>2015-07-15T23:19:00.000Z</dateUtc〉

$</$ actualGateDeparture>

- <estimatedRunwayDeparture>

〈dateLocal>2015-07-16T09:39:00.000</dateLocal> 〈dateUtc>2015-07-15T23:39:00.000Z</dateUtc〉

</estimatedRunwayDeparture>

- <actualRunwayDeparture>

〈dateLocal>2015-07-16T09:39:00.000</dateLocal〉

$\langle$ dateUtc>2015-07-15T23:39:00.000Z</dateUtc>
$</$ actualRunwayDeparture >

- 〈scheduledGateArrival>

〈dateLocal>2015-07-16T14:15:00.000</dateLocal>

〈dateUtc>2015-07-16T02:15:00.000Z</dateUtc〉

$\langle/$ scheduledGateArrival $\rangle$

- 〈estimatedGateArrival>

〈dateLocal>2015-07-16T14:29:00.000</dateLocal> $\langle$ dateUtc〉2015-07-16T02:29:00.000Z</dateUtc〉

</estimatedGateArrival>

- 〈actualGateArrival>

〈dateLocal>2015-07-16T14:29:00.000</dateLocal〉

〈dateUtc>2015-07-16T02:29:00.000Z</dateUtc>

$</$ actualGateArrival>

- 〈estimatedRunwayArrival>

〈dateLocal>2015-07-16T14:25:00.000</dateLocal〉

<dateUtc>2015-07-16T02:25:00.000Z</dateUtc>

</estimatedRunwayArrival>

- 〈actualRunwayArrival>

〈dateLocal>2015-07-16T14:25:00.000</dateLocal> <dateUtc>2015-07-16T02:25:00.000Z</dateUtc〉

$</$ actualRunwayArrival>

</operationalTimes >

- 〈codeshares>

- <codeshare>

$\langle$ fsCode $>\mathrm{DL}</$ fsCode $\rangle$

〈flightNumber>7235</flightNumber>

$\langle$ relationship $\rangle$ L/relationship $\rangle$

$</$ codeshare $>$

- <codeshare>

$\langle$ fsCode $>E Y</$ fsCode $>$

〈flightNumber >6694</flightNumber >

$\langle$ relationship $\rangle$ L $/$ relationship $\rangle$

$</$ codeshare $>$

- <codeshare>

$\langle$ fsCode $>\mathrm{NZ}</$ fsCode $>$

<flightNumber >7914</flightNumber >

$\langle$ relationship $\rangle$ L/relationship $\rangle$

$</$ codeshare $>$

- <codeshare>

$<$ fsCode $>$ SQ $</ f s C o d e>$

$\langle$ flightNumber $>6522</$ flightNumber >

$\langle$ relationship $\rangle$ L $<$ relationship $\rangle$

$</$ codeshare $>$

- <codeshare>

$\langle f s C o d e\rangle V A</ f s C o d e\rangle$

<flightNumber >104</flightNumber >

$\langle$ relationship $\rangle$ S $</$ relationship $>$

$</$ codeshare $>$

$\langle/$ codeshares $>$ 
Each point is a passenger (data from aircraft type)
Points move randomly around the 'world' but group with points from the same flight

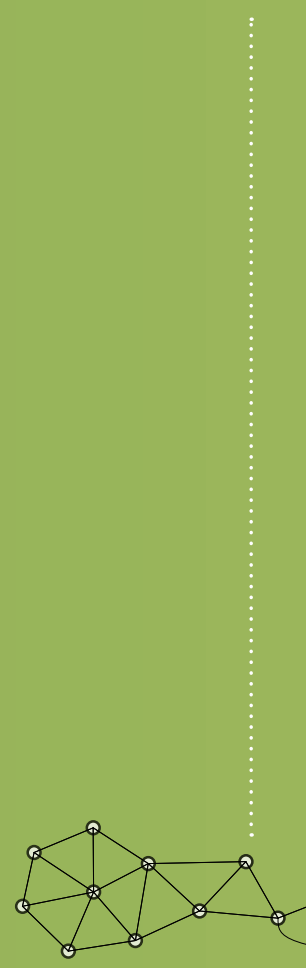

$\circ$

$\circ$

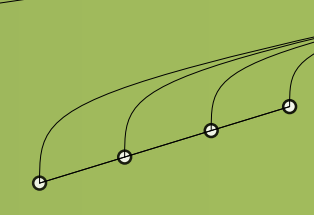

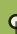




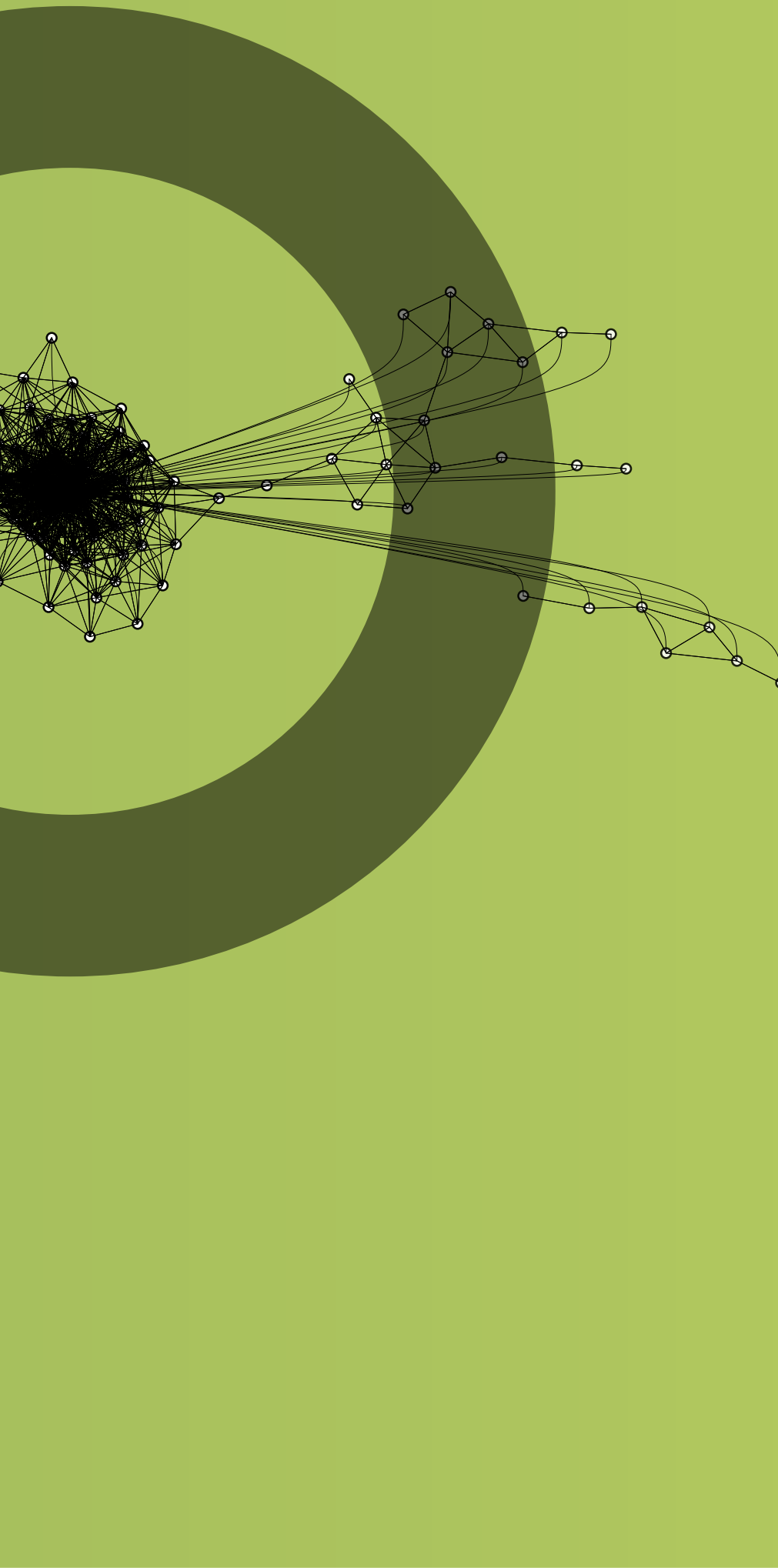

3.06 Princemio's Pathfinder is visual software which inspires dancer's physical movement, in the same way the project attempts to represent mass users and their movements.
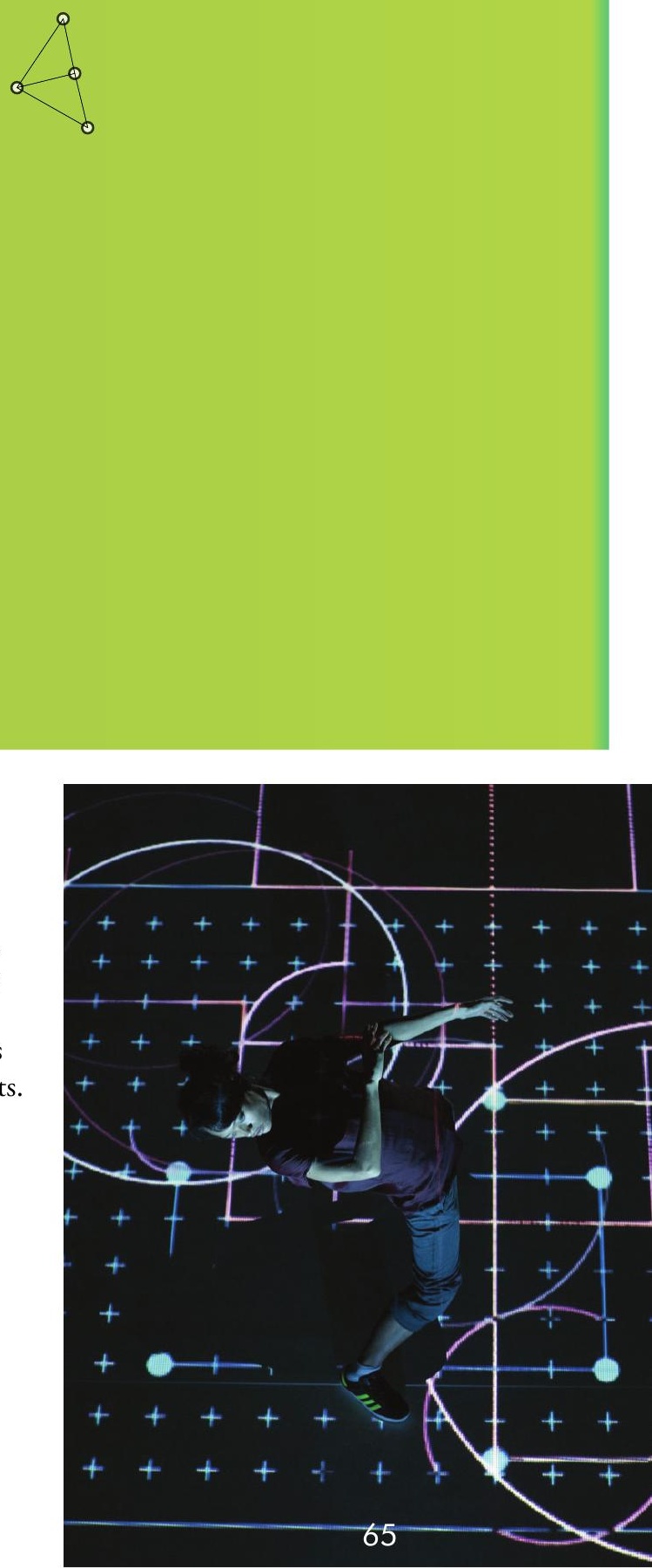


\subsubsection{USER SELECTIONS}

An experiment using Processing library ${ }^{3}$ ControlP5 allowed the programme to allow selection of flights through a drop down menu. The selection causes the points for a flight to increase in radius, allowing an initial level of personalization. However, it causes issues with multiple users looking at the program at once. It also helps with the readability of the spatial representation.

3 Processing libraries are third party plug-ins to add functionality, ControlP5 allows UI elements.

\section{display( );}

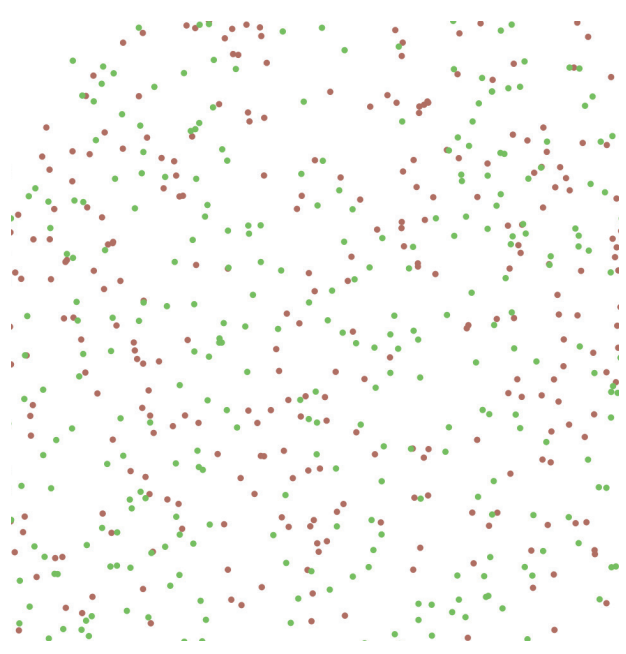

3.07 Initial Processing sketch, inputted number of points and flights is displayed with a random colour assigned. The points move randomly on surface. This was a test for Processing.

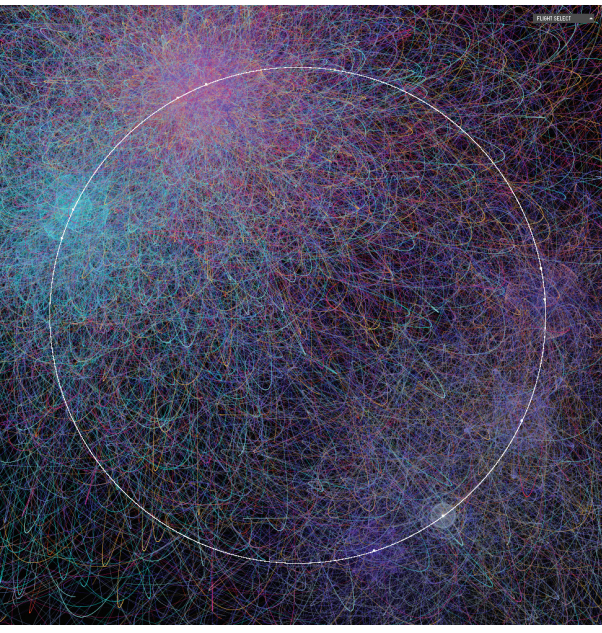

3.08 A test where trails are left behind points, an attractor pulls points closer, creating areas of activity around the attractor when flights are close to departing. Attractor points map to gates in physical space. 


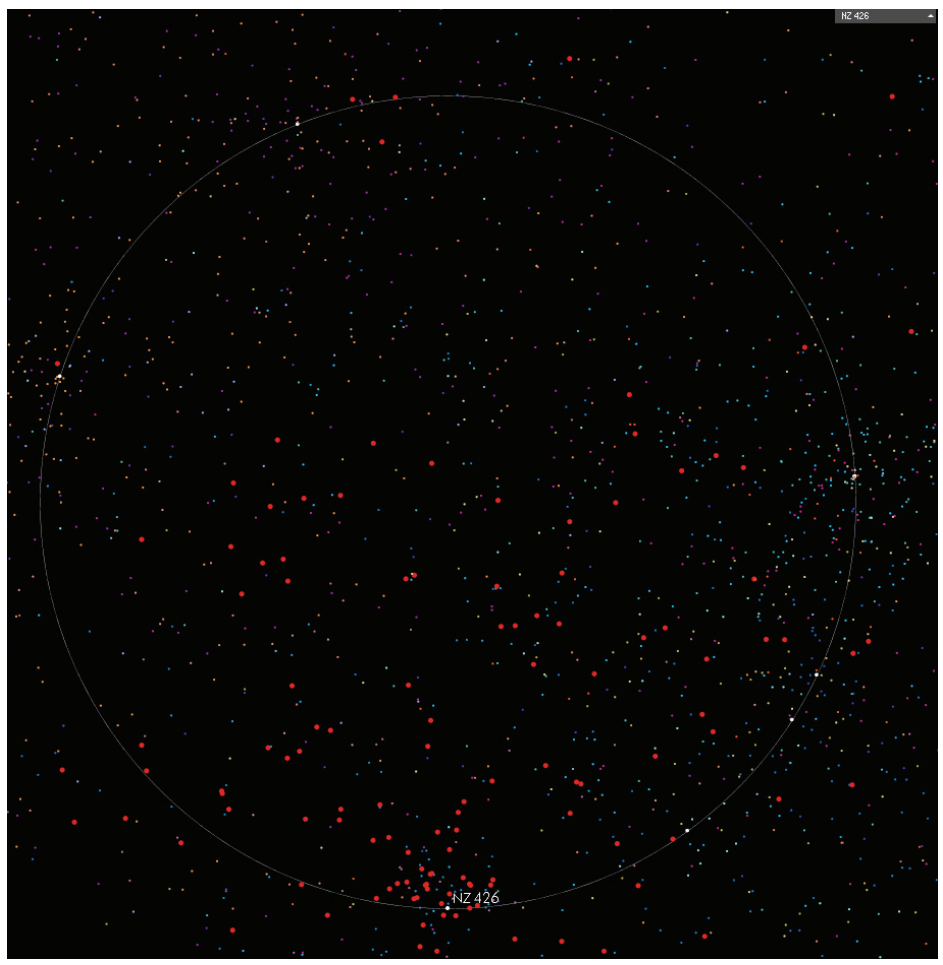

3.09 A Flights' points become bold when selected from a drop down menu by a user, allowing customisation by flight. 


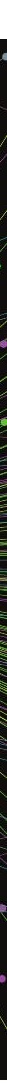

3.11 Attractors draw together points visually, creating clusters of colour. Attractors are arranged in the same order as in physical space. 


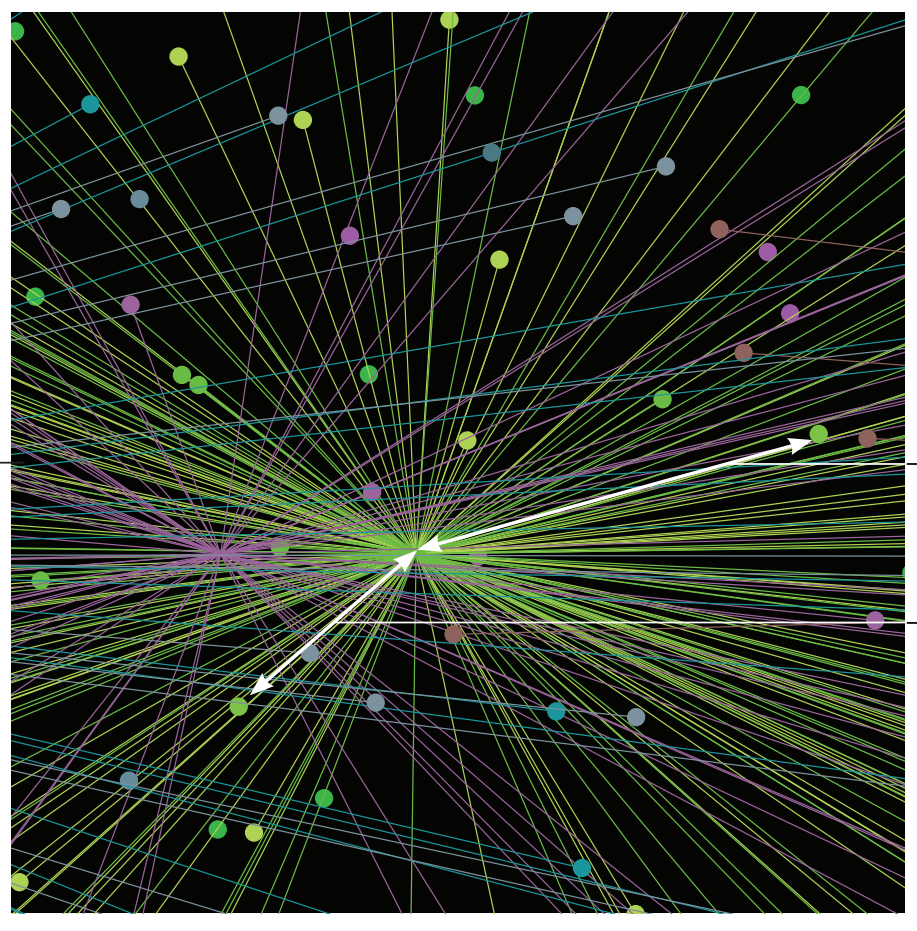

3.10 Lines connect points to attractors, beginning to show pathways in physical space. Speed and acceleration depends on distance to attractor.

distance $=100$

attraction acceleration $=0.2 \mathrm{px} /$ frame

distance $=20$

attraction acceleration $=1 \mathrm{px} /$ frame

$\operatorname{attractor}(a)$;

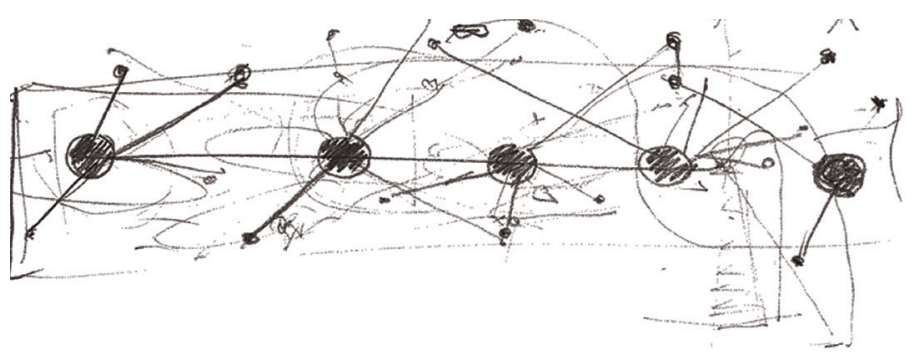

3.12 Sketch of initial concept of visual flight data organisation. 


\subsubsection{FLOCKING ALGORITHM}

The initial logic provides a framework for spatial understanding of people moving through space based on actual data. However, this doesn't provide a realistic representation of users' movement; there is no interaction between the points. Reynolds' flocking algorithm (2015), with a provision of cohesion, separation and alignment, simulates movement of users through space ${ }^{4}$. This is done so all points interact with each other, or so just points on the same flights interact with each other. Lines between points on the same flight groups people visually.

4 This behaviour is shown in many living beings such as birds, fish, bacteria, and insects, and can be abstractly applied to groups of humans.

3.13 Flocking creates groups of points that are not always around the attractor point, but instead clusters that move independently. Lines connect groups when the distance between is below a set value. 


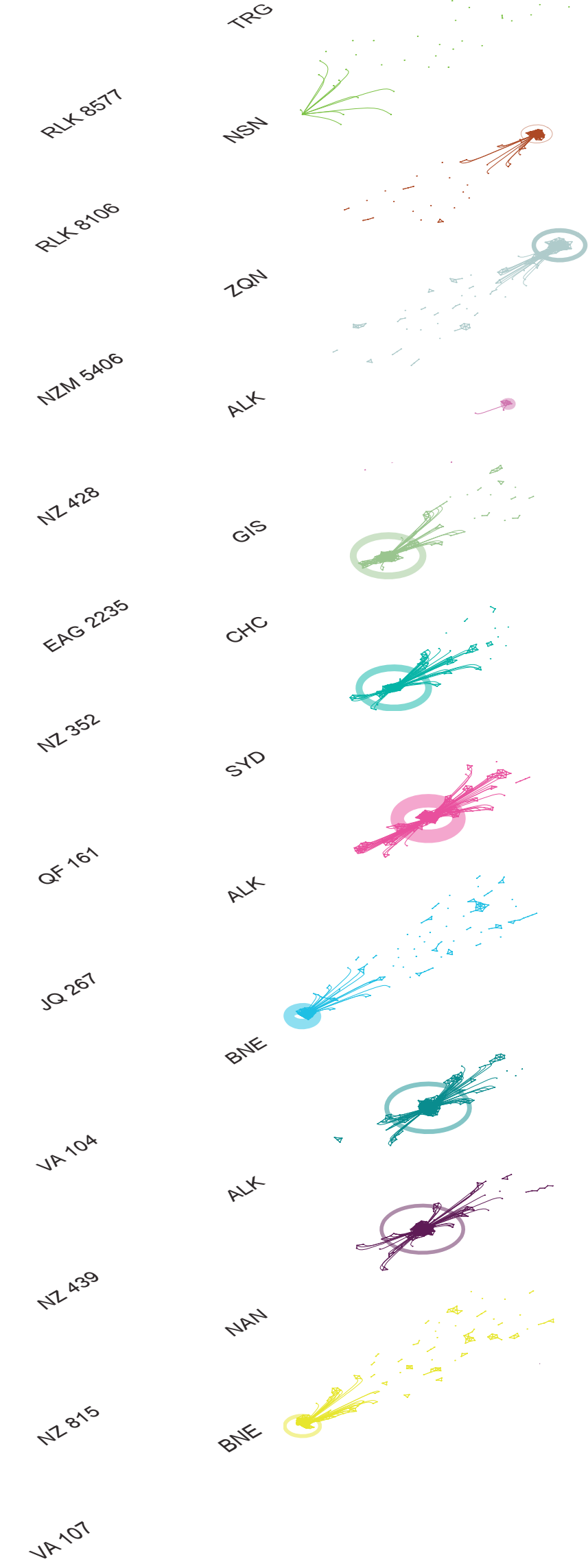

3.14 Exploded view of the visualisation for each flight. Attractor points remain mapped to the gate locations in the physical architecture. 


\subsubsection{INTERPRETATION}

Location of individuals as points provide detailed information of the social movement of users, but information needs to be conveyed quickly and at a glance. Interpretation produces a clear set of information that isn't seen in the raw data inputs (Achten, 1997). To understand the location of groups of people, overlays of hues show groups of 10 or more people within a set radius on the same flight. This awareness visually notifies users when they are in 'the pack' or falling behind in a spatial way, forming a 'smartmob' enabled by computing (Shekhar, Feiner, \& Aref, 2015).

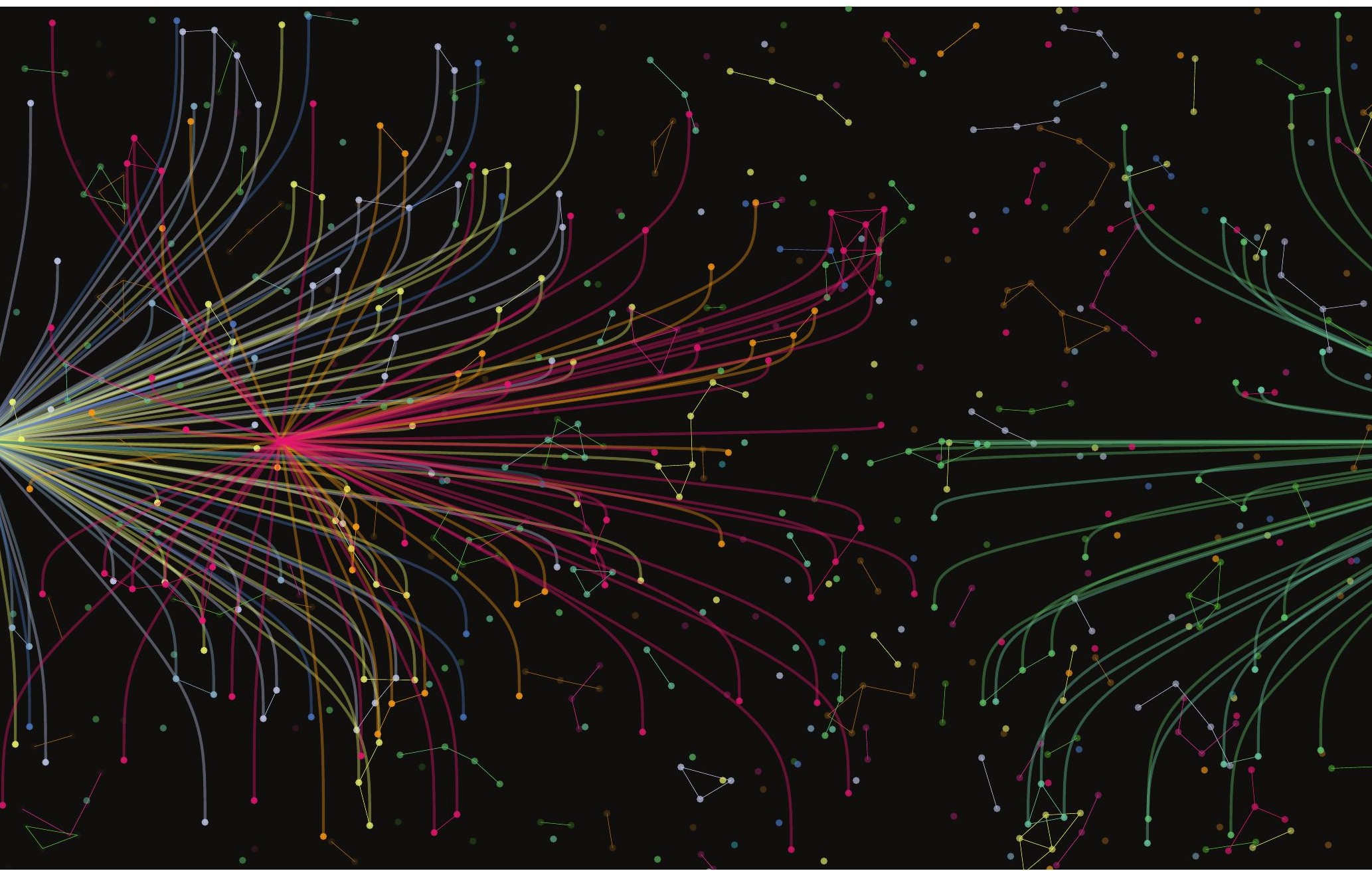

3.16 Overlays colour the visualisation lightly while keeping the dense data visible. The amount of users/points close to the attractor informs a circle around attractor. 


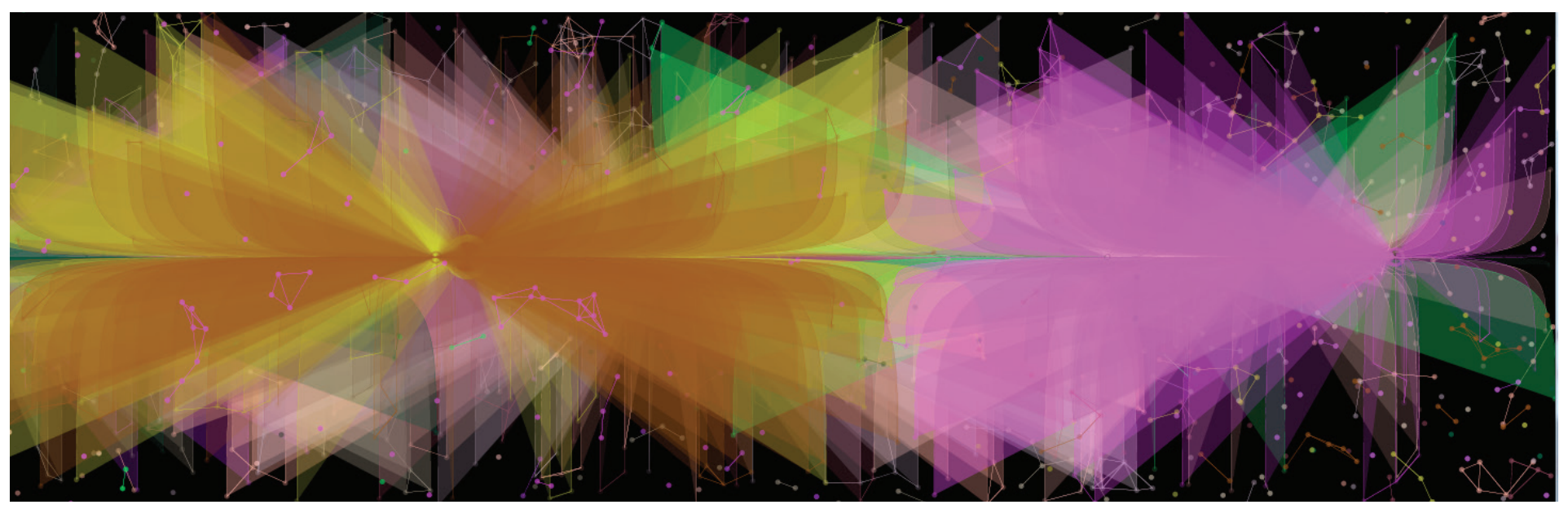

3.15 Shapes are created between points and attractors with a transparency, this pattern is both aesthetic and functional showing density around certain areas.

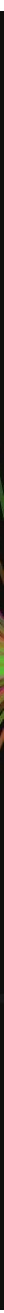




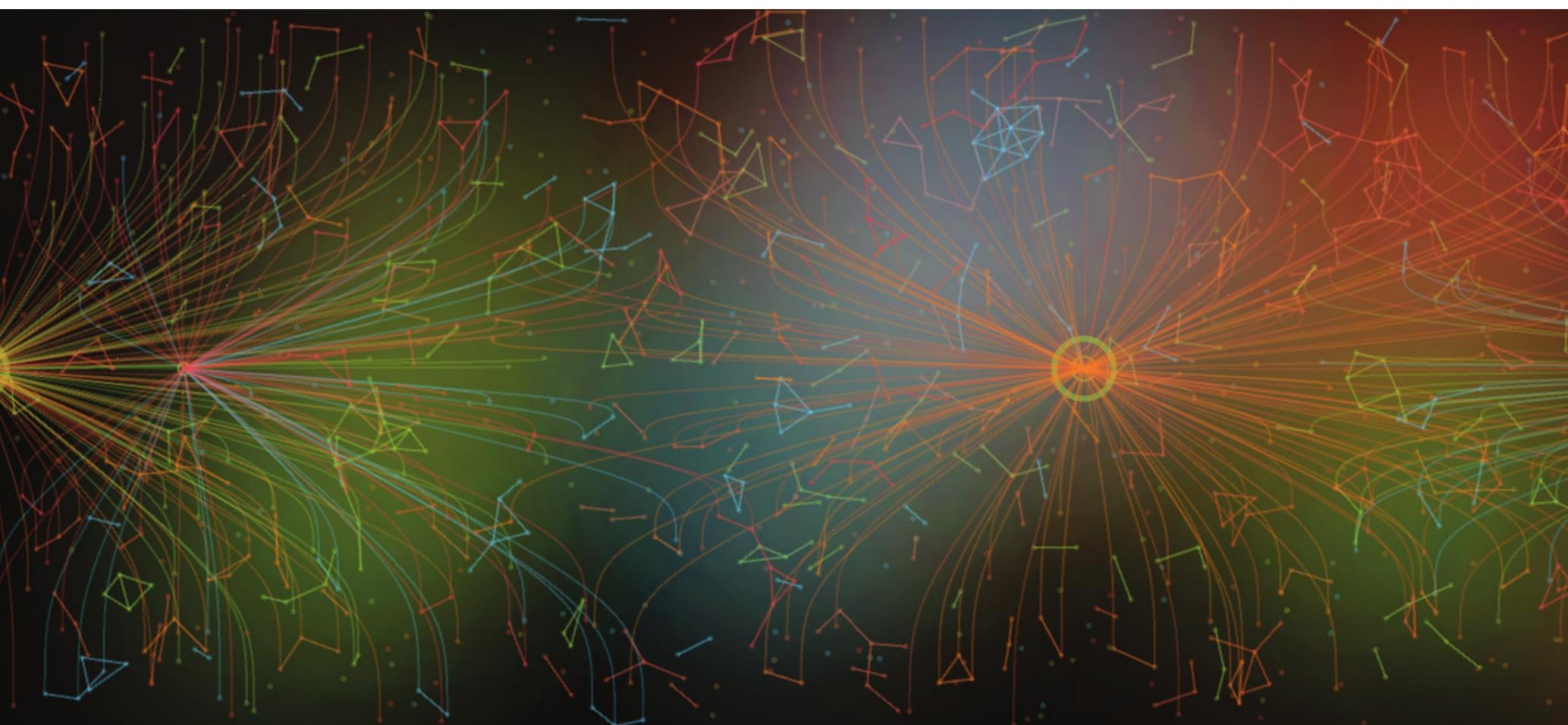

3.17 A hue covers areas of dense points, no matter where they are in the space - the brighter they are, the more people are there. This is a visual tracking of users through the airport.

$\longrightarrow 3.18$ Movement of the visualisation across various times of the day, in $10 \mathrm{~min}$. intervals. 


\title{
DESIGN REVELATION
}

\author{
- LOCATION -
}

It became apparent that the location of the user and architecture in the system was always going to be key to being successful. The relationship between these needs to enforce context and give representations of position, activity and organisation.

\author{
IF ARCHITECTURE AND INTERACTION \\ DESIGN ARE TO BENEFIT ONE ANOTHER AS \\ DISCIPLINES, THEY MUST WORK TOGETHER \\ ON LOCATION MODELS.
}

- MCCULLOUGH 


\subsubsection{VISUALISATION ON SPECIFIC}

\section{ARCHITECTURES}

The Architectural Representation is a simulation of a plan of the space, but doesn't denote specific points in space. There needs to be a context specific visualisation within the space, providing an extension of 'coherent spatial experience' (Anders, 2007) through a logical and simple methods. Based on the initial programme, an additional function calculates the distance of interpreted points to a set point in physical space, creating a simulated, augmented, panoramic view from that point. When a physical point is located close to a gate and that gate is boarding, the visualisation becomes the colour associated with that flight.

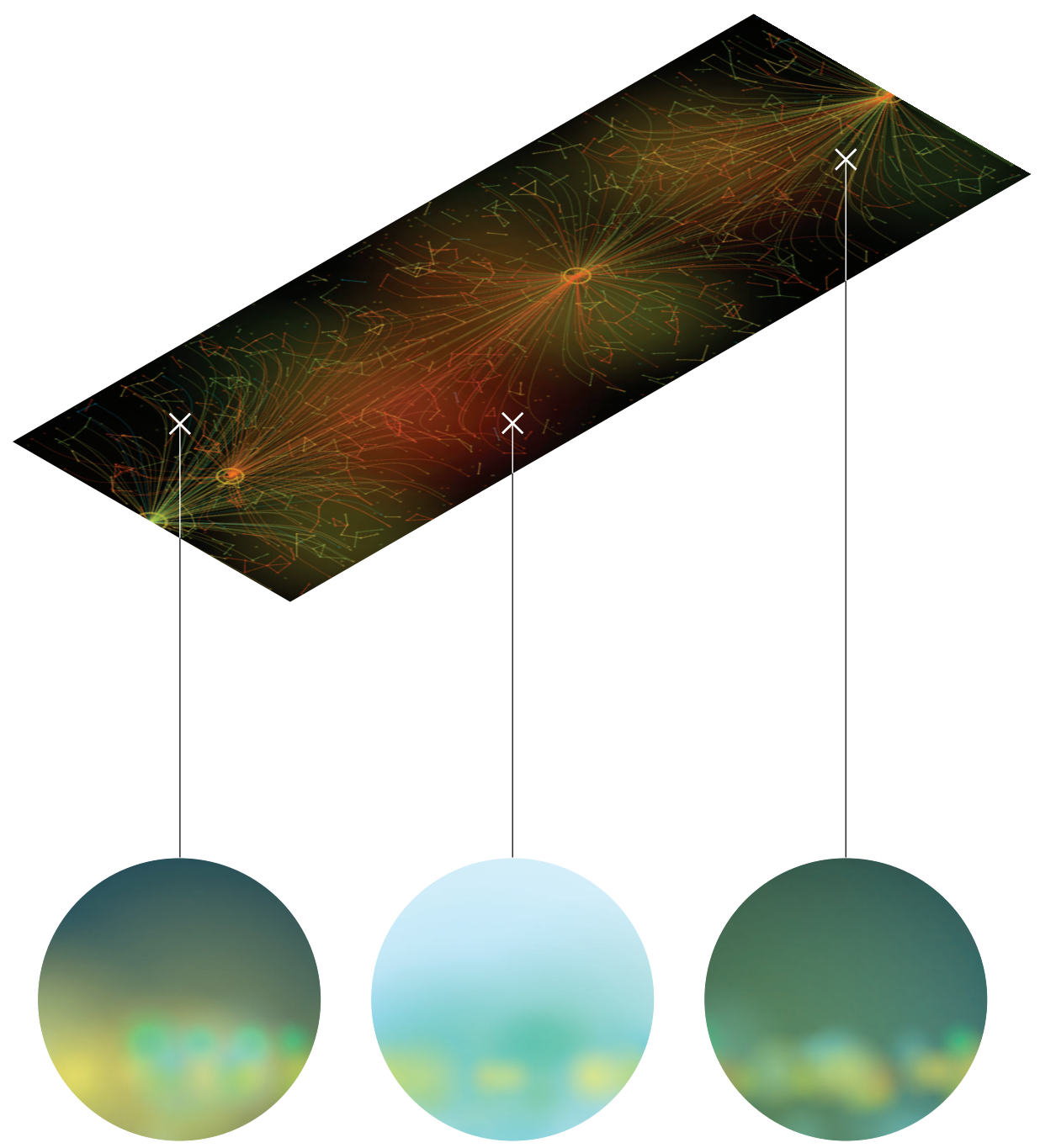

3.19 Abstractions at different points from the same projection produce context and time specific visualisations, which can be displayed on architectural forms. 

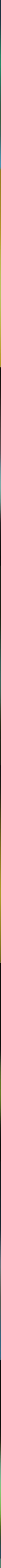


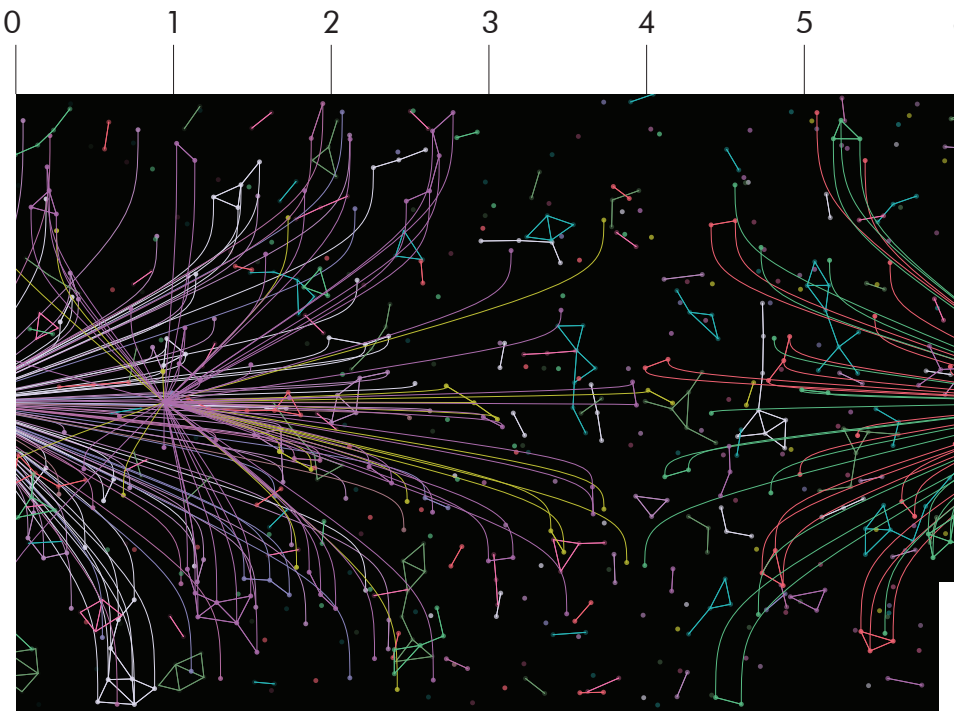

THE LOCATION DRAMATICALLY INFLUENCES THE OUTPUT, JUST AS MUCH AS THE RAW DATA. THIS TELLS A STORY ABOUT THE DATA LANDSCAPE, OFFERING A VIEWPOINT INTO THE WORKINGS OF PASSENGER FLOWS.

4 3.20 Movement of the abstracted space specific visualisation points across the simulation of space.

$9 \$ 3.21$ Locations of the spatial interpretation on the base visualisation. 
BOARING PASS SCANNER

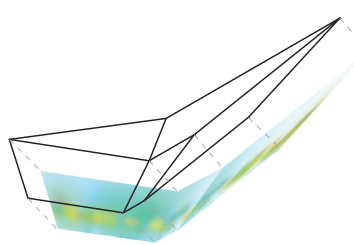

FOOD STALL PAVILION

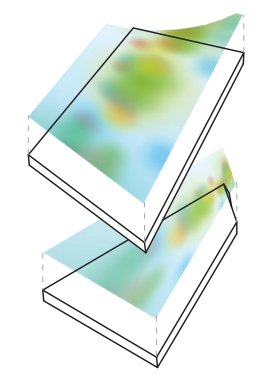

BAGGAGE CAROUSEL
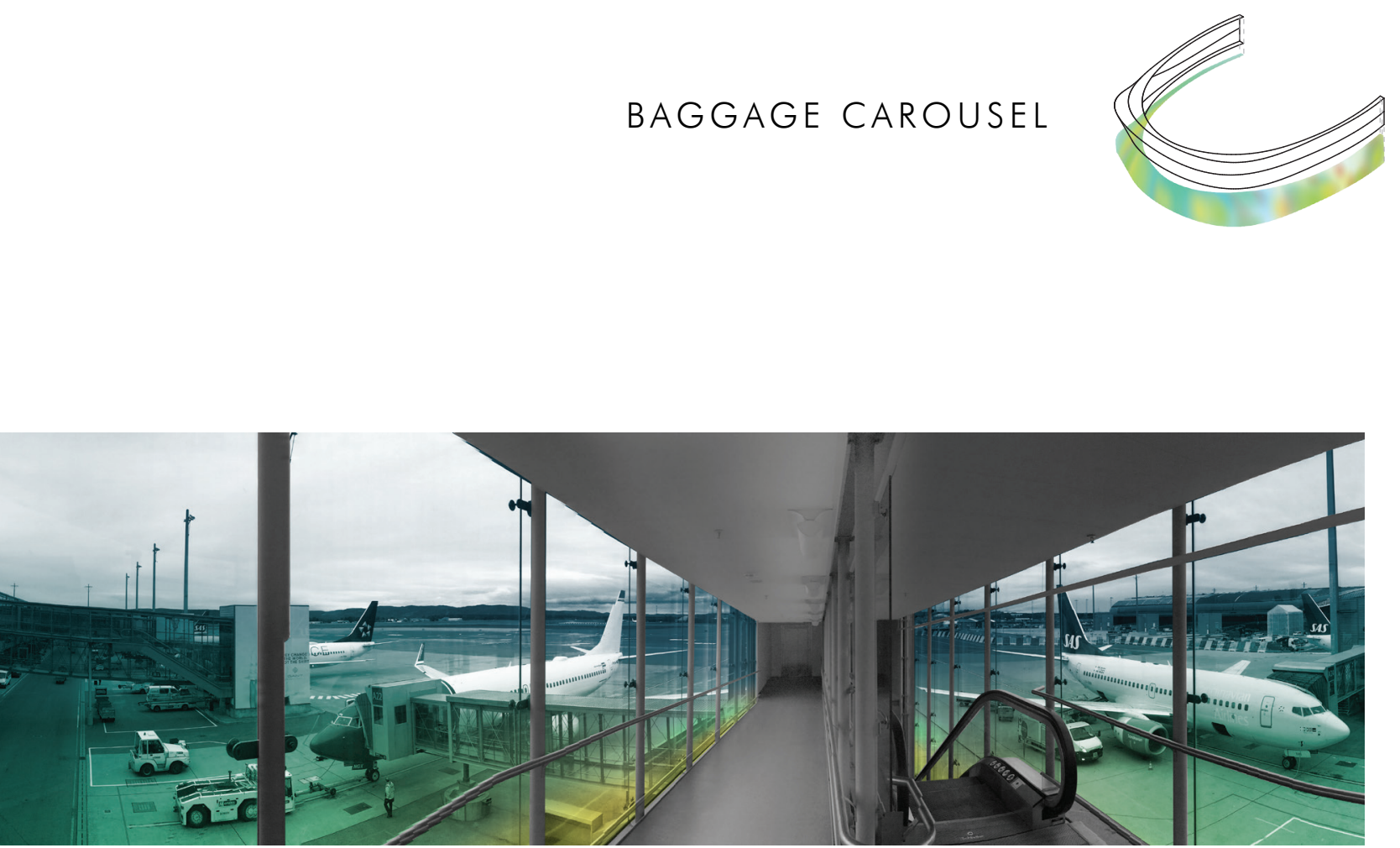

$\triangle$ 3.22 Movement through the airport by users is a series of decisions at key wayfinding points.

The physical designs at these points can then have virtual displays on them, helping users navigate. Objects become a network of signage systems, allowing a constant awareness of the social movement taking place.

\ 3.23 Lighting can also be used to reassure passengers of their flight, for example the air bridge. 


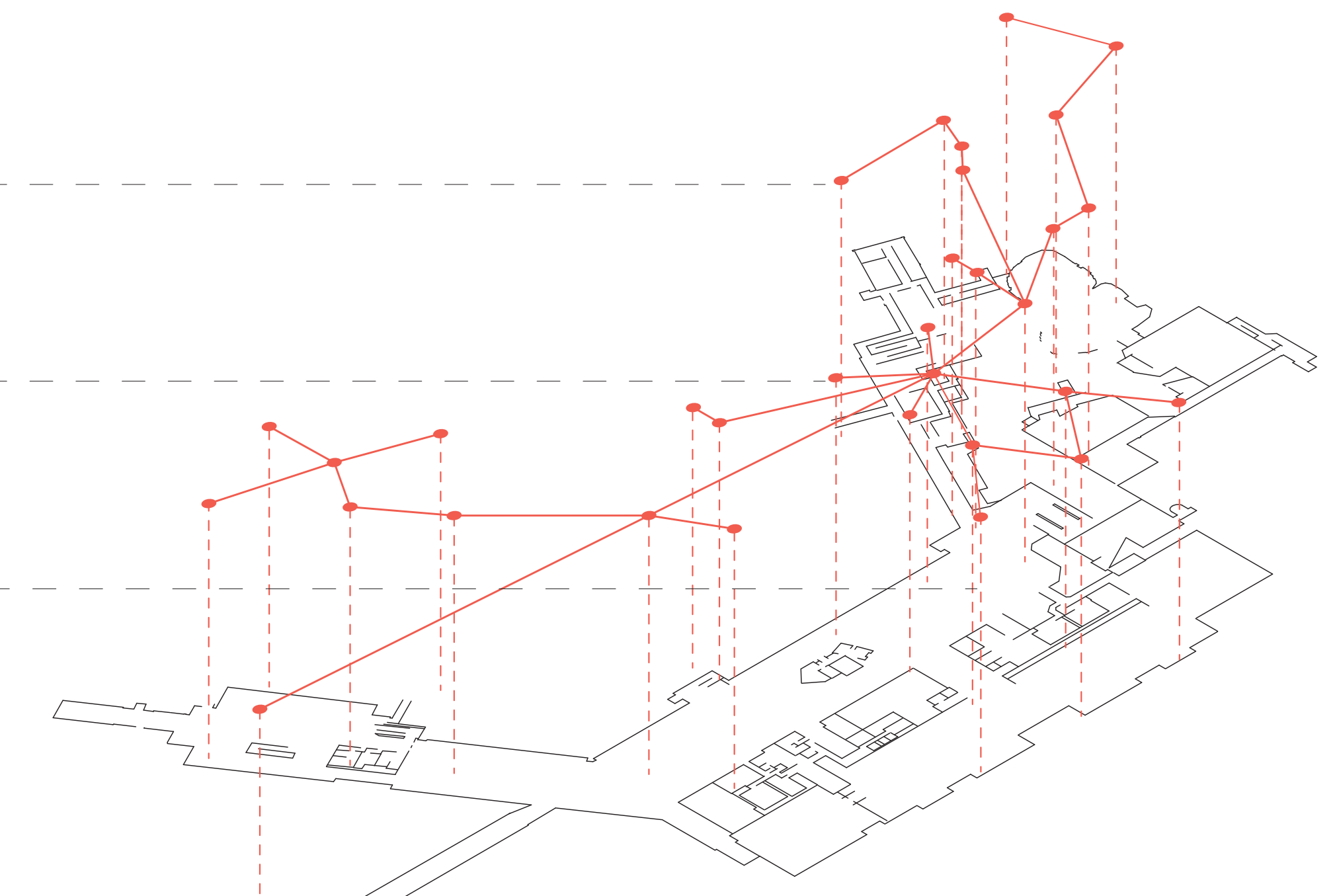




\subsubsection{SUMMARY}

Through developing a visualisation, a kinetic virtual form is produced through interactions using data sources and introducing various technologies important to the data source. There are little issues with mechanical movement, using light as a material. The notion of a data landscape is introduced (fig. 3.19), covering large spaces and representing the amounts of users in a space. To create further engagement, physical forms need to be designed. 


\subsection{MIXED REALITY}

As a dual real virtual creator of spatial experience, the form is important in the creation of spatial experience. Like real-time data, there is also data about places that don't change often, which is still about the users. This informed a series of experiments with the translation of data to $3 \mathrm{D}$ forms, which are then built.

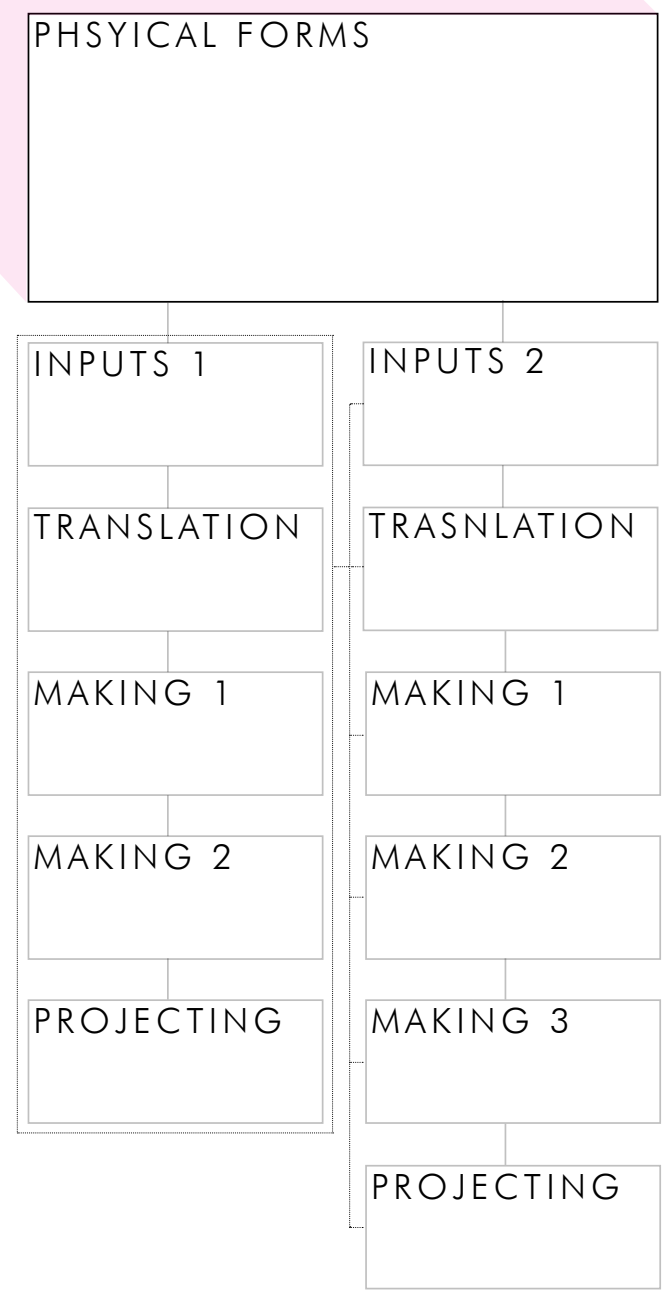




\subsubsection{FORM PROTOTYPE 1}

A compounded data set of distance, flight time, duration, flight amounts and passenger numbers for each destination from Wellington Airport ${ }^{5}$, formed a set (fig. 3.25) that could be transformed using the layout of the existing airport to map gates to directions. This resulted in a series of interactions, produced in grasshopper, which represent averages of flights from the airport in a single form (fig. 3.27). Where destinations are further away, more space is created inside the form, and where the distance was shorter, the forms are smaller. This provides a form to projection map spatial simulations onto, where the attractor points of gates are situated at the end points of the form. This connection made both virtual and physical forms have further depth of understanding.

5 Data sources are from Wellington Airport Airline websites, collated by the author.
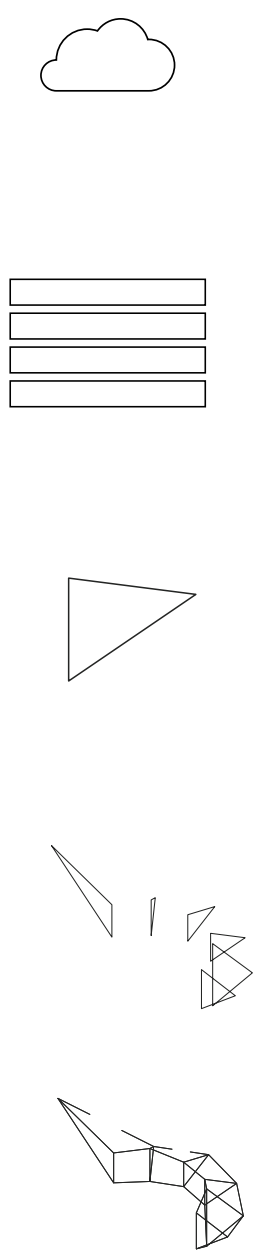

1 INPUT

Data is assembeled in a spreadsheet.

2 PARSING

Grasshopper reads data and creates lists of data types
3 TRANSLATION

Each variable is transformed from a central point to create triangular shapes for each gate

4 GATE TRANSLATION Each form is rotated to face the gate to which it corresponds

5 FORM CREATION

Between each data types node and location, a line is created to form a mesh.

3.24 Method of translation for Physical Form 1 using the Wellington Airport Flight Data. 


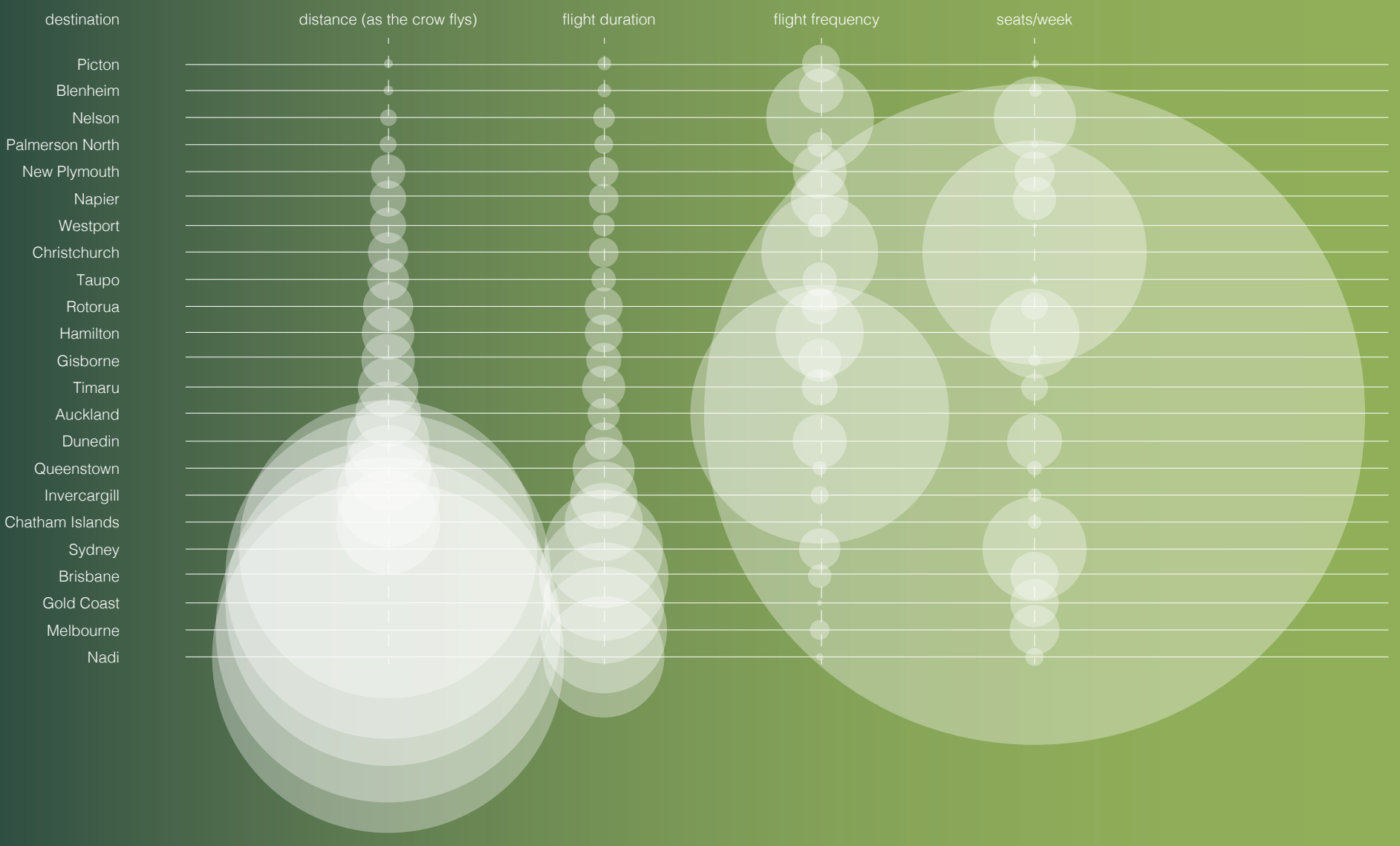

3.25 Graphical representation of Wellington Airport flight data, displaying connections between destination distance, time and flight capacity. 


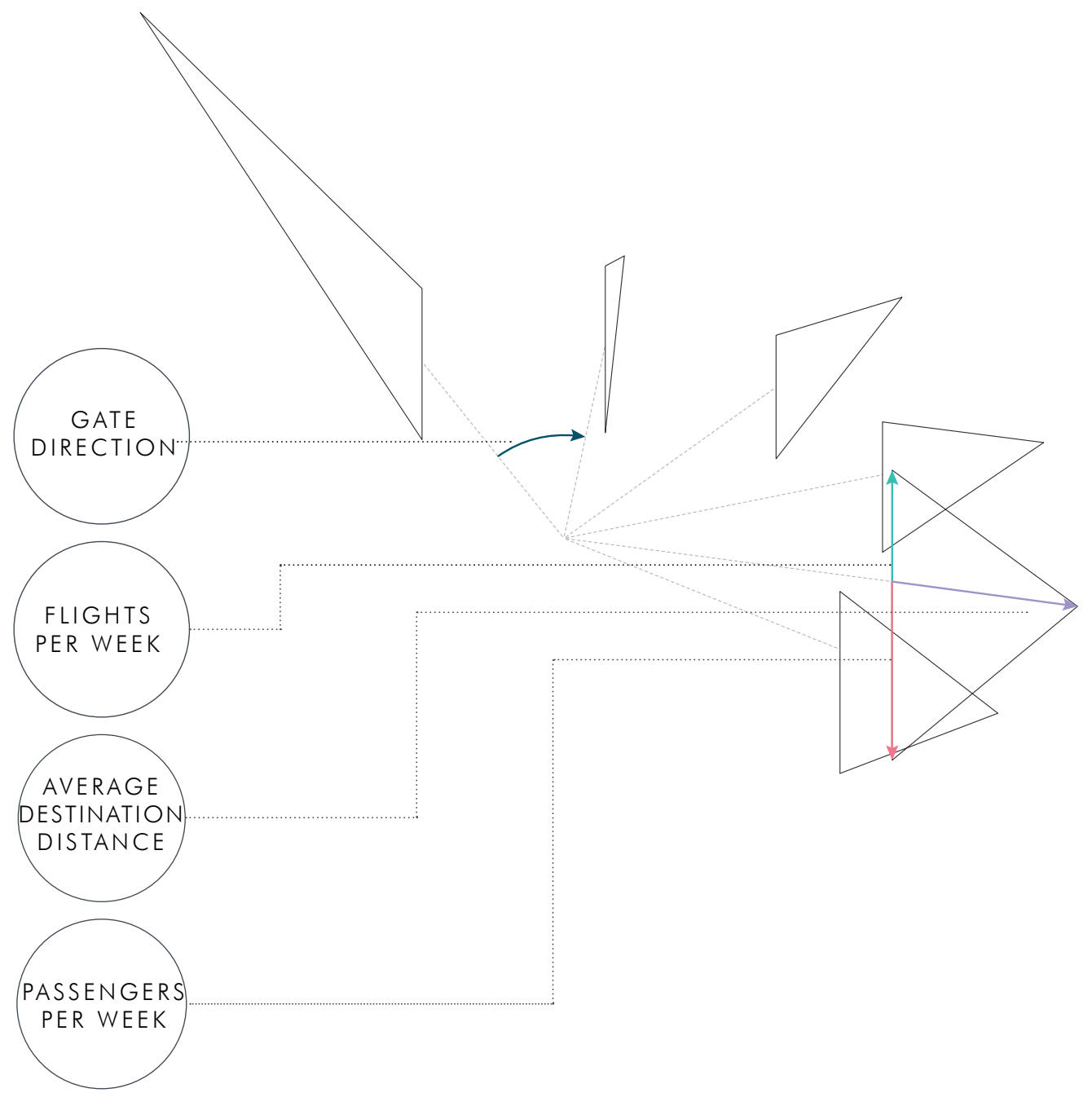

3.26 Location of embedded data within the form. 

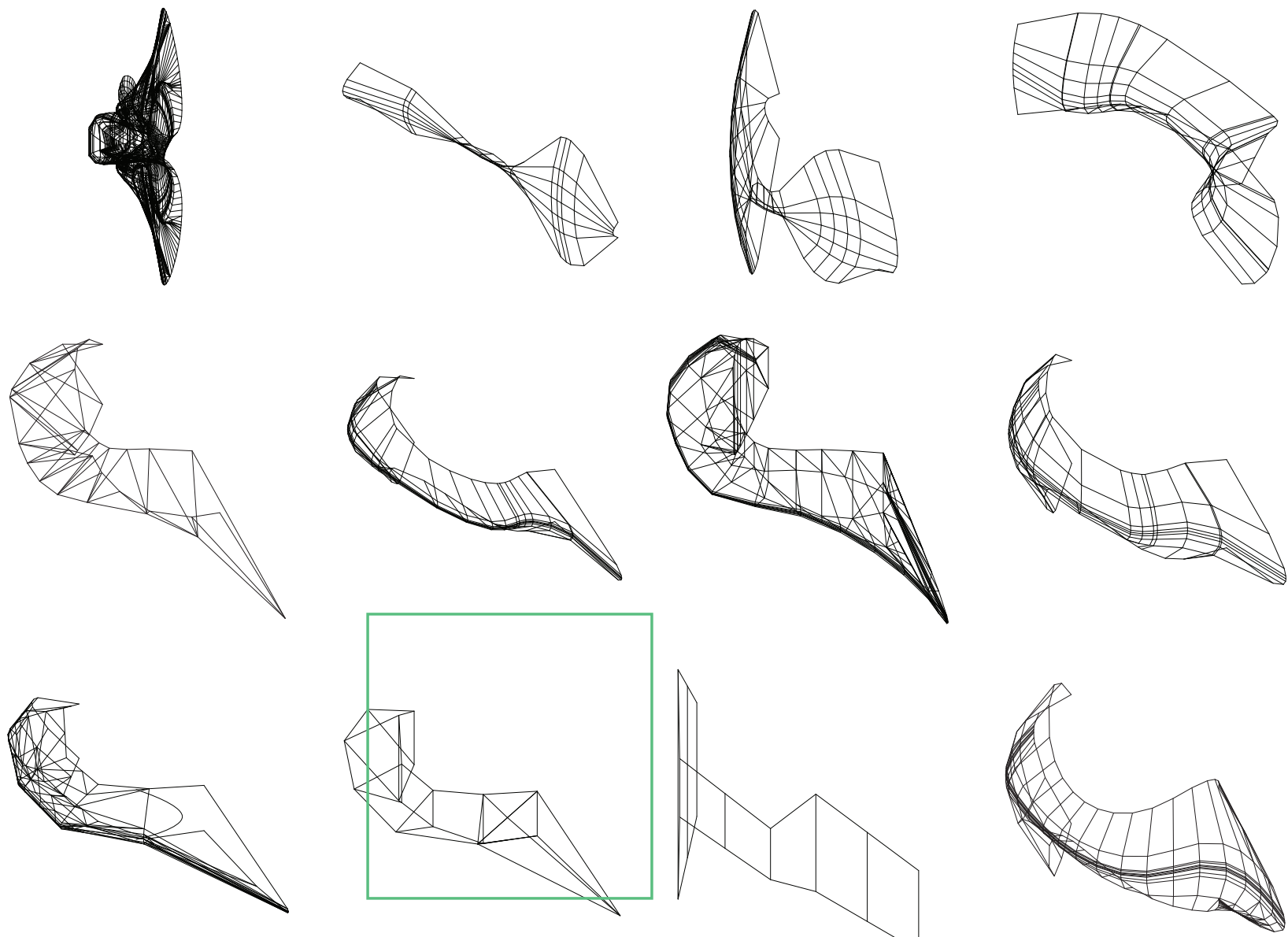
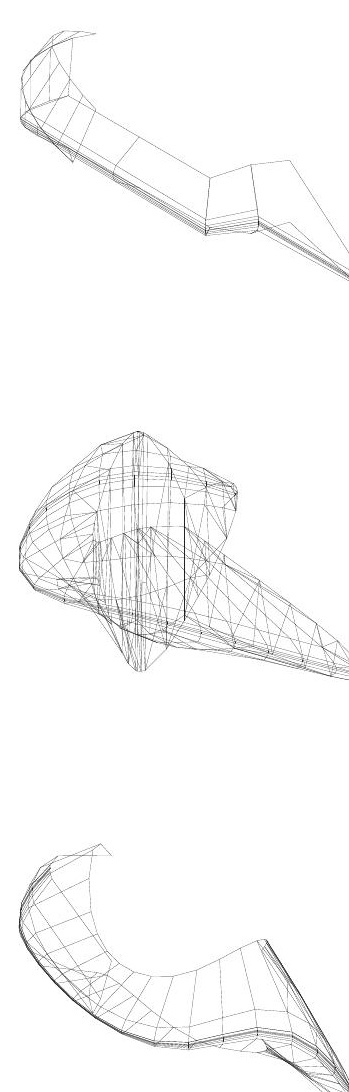

3.27 Outputs representations by tweaking the grasshopper logic.

This resulted in both smooth double curved and faceted surfaces. A

simplified form was chosen for construction. 

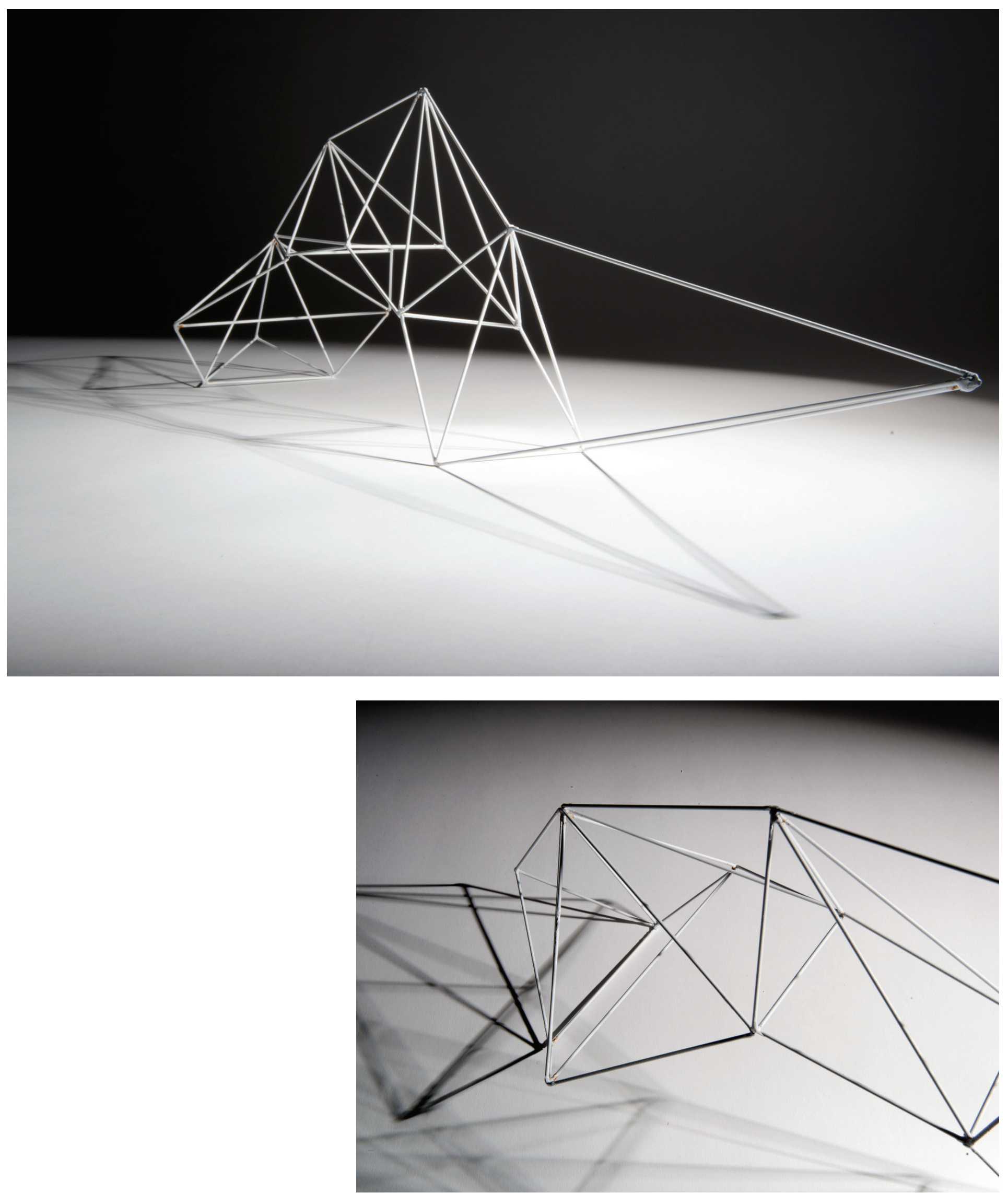


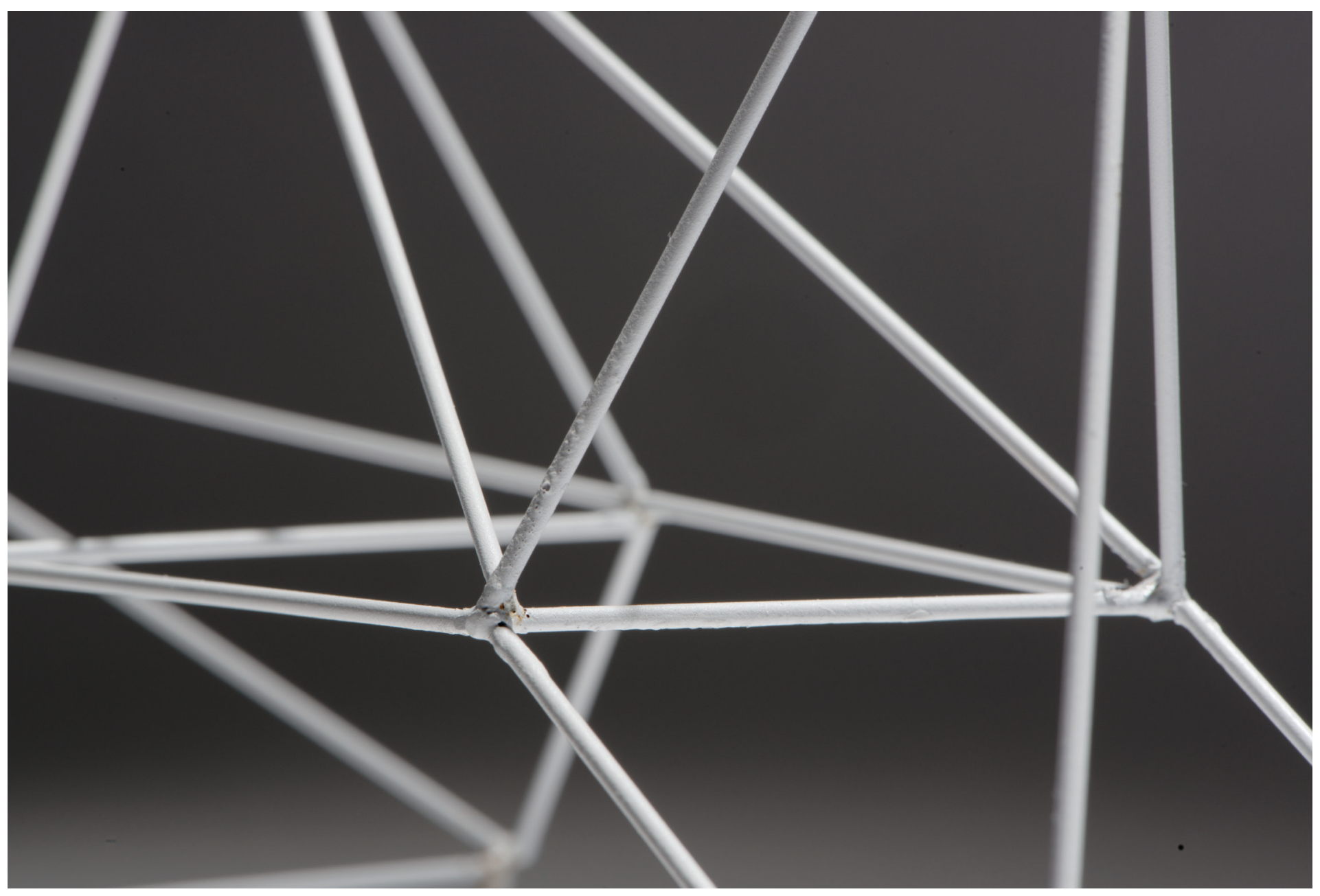

\section{WIRE}

- 3.28 Manually constructed form using soldered copper rods with white matte paint.

$\longleftarrow 3.29$ Shadows cast from the wire form. The construction method implies full scale construction methods and an interior form similar to Salt Water Pavilion.

^ 3.30 Connections details. 


\section{PAPER}


4 3.31 Paper folded form created using a data output from grasshopper and Autodesk 123D make.

$\checkmark$ 3.32 The location of data is aimed to be seen throughout the form. Form highlights key data points within the visualisation, such as larger international departure areas. 


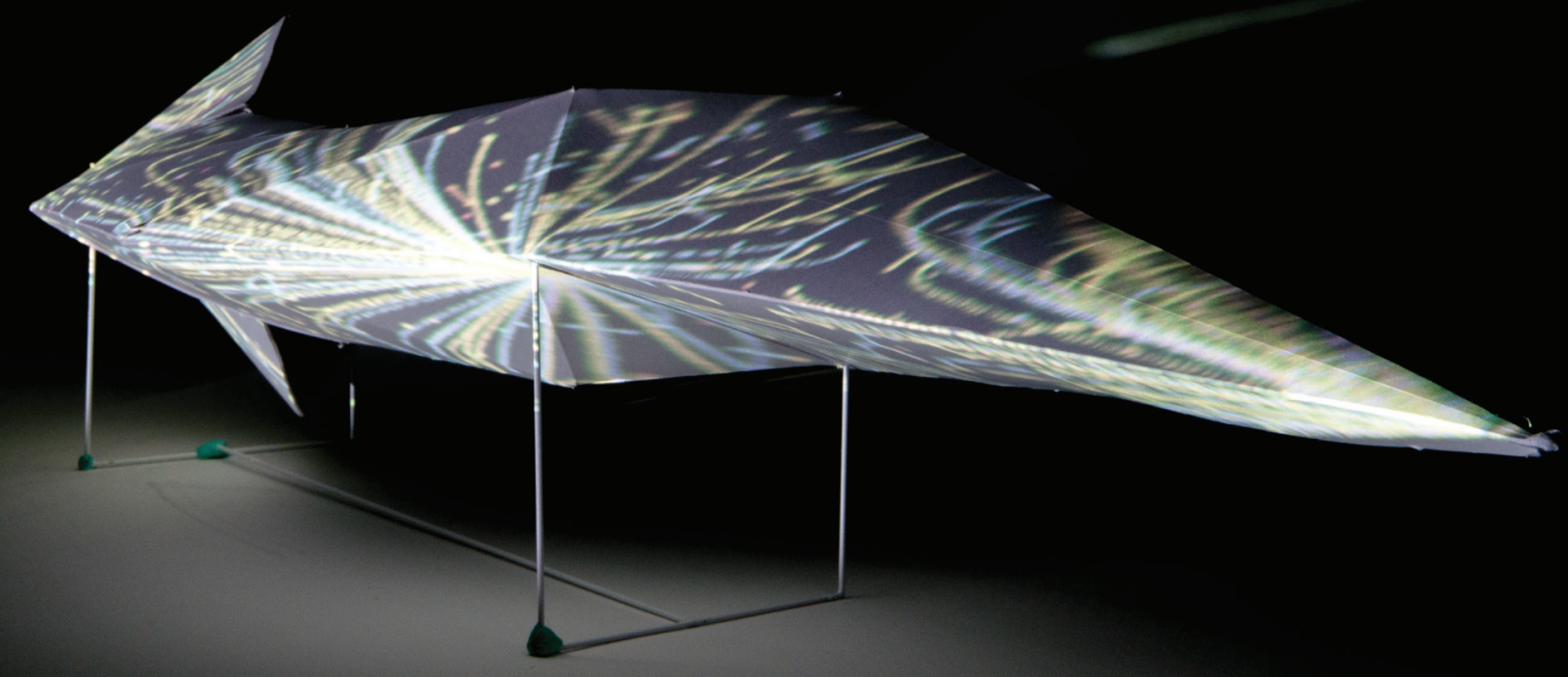

9)

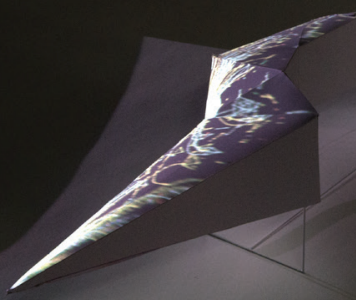




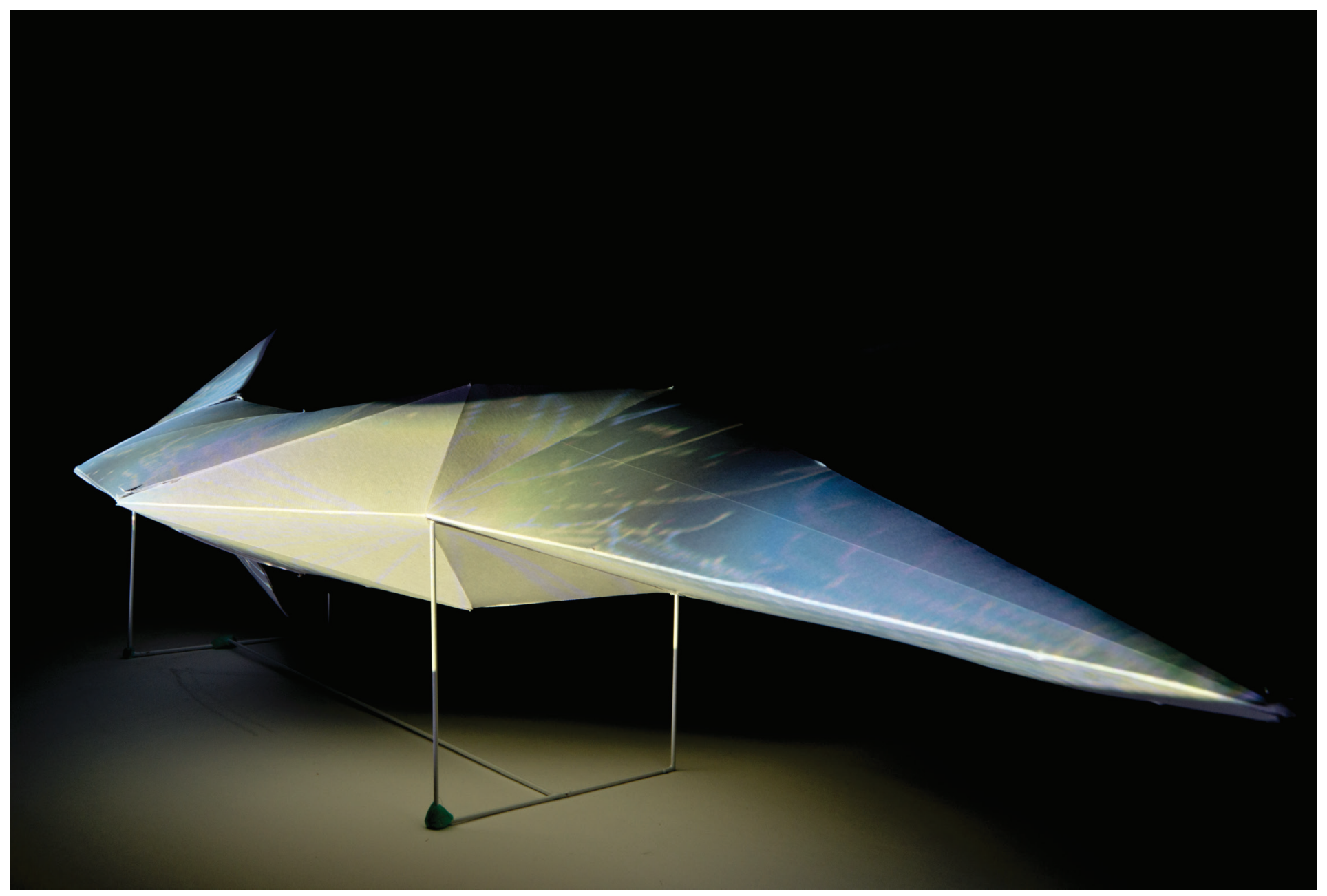

\section{PROJECTION MAPPING}

- 3.33 Attraction Projection. Projection onto paper folded model maps to the surface; each medium highlights details within the other.

3.34 Projection mapping setup.

4 3.35 Trends' projection allows a further removed form. There are issues with allowing passengers to read the system easily and coherently.

$\rightarrow 3.36$ Ascent, by Nocte, is a digital sculpture to link people to landscapes. Appearance of form dynamically changes depending on virtual inputs.

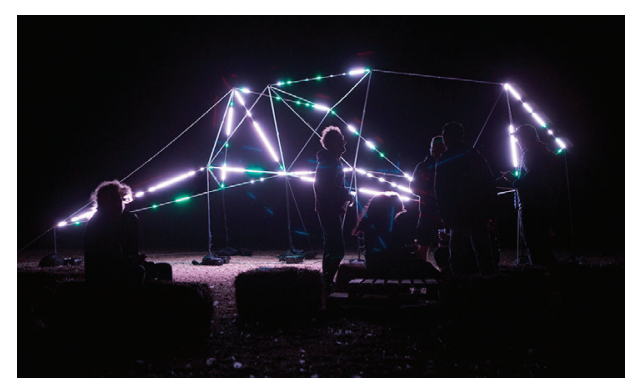


3.37 1:200 Latitudinal Section. Space created by the form, including the ability for it to become a wayfinding system, is visible throughout the space.

3.38 1:200 Latitudinal Section. Inhabitation of form suggests the ability for an enveloping experience from within, similar to Salt Water Pavilion.

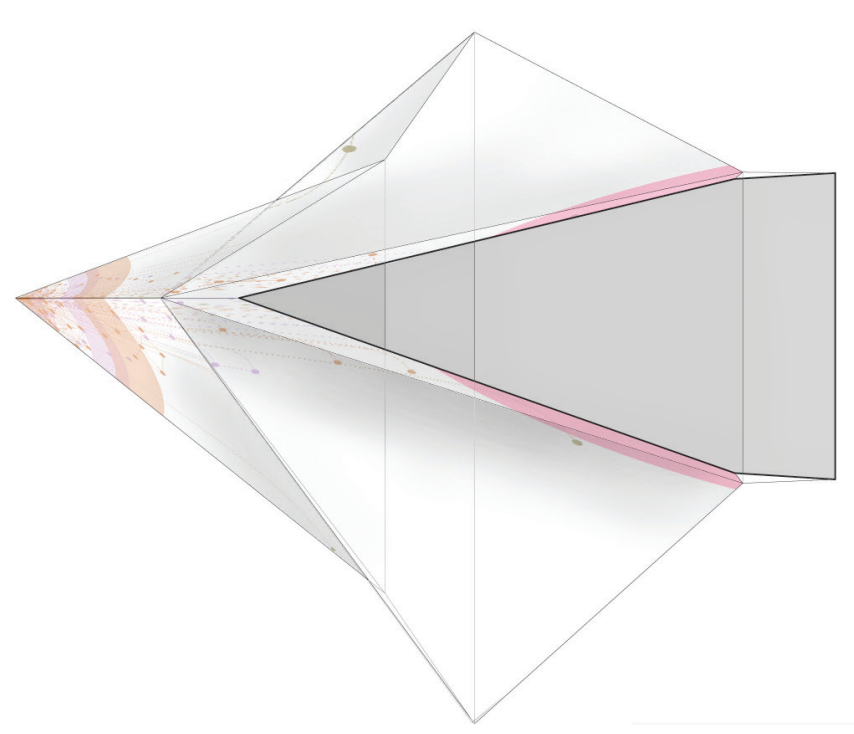

3.39 1:200 Longitudinal Section.

Connections to virtual simulation through projection on form. The architectural scale object becomes a wayfinding device. 

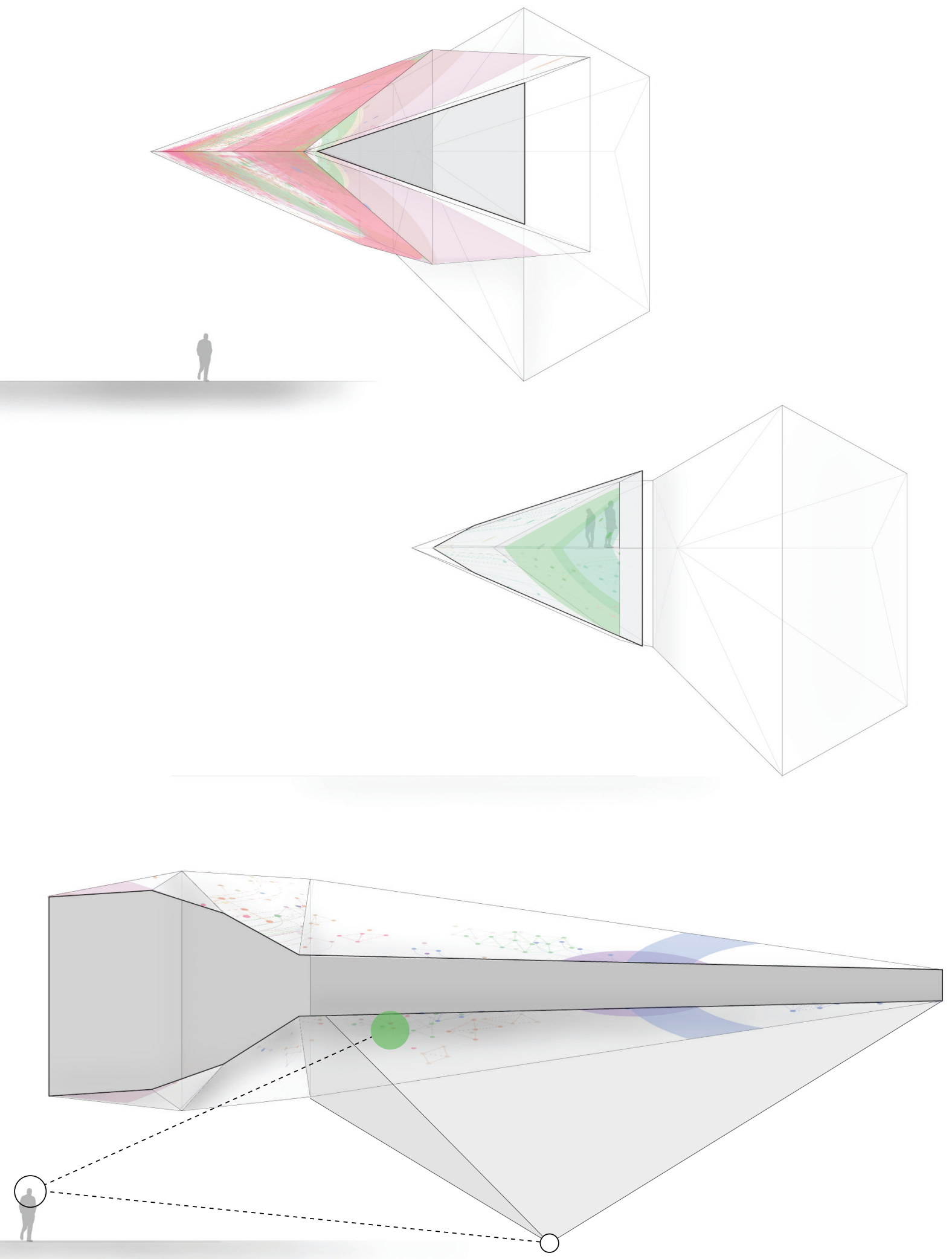


\subsubsection{FORM PROTOTYPE 2}

This second experiment is constructed from Airport Visitor Survey Data ${ }^{6}$ (fig.3.41). This data was chosen because of its high level of detail across multiple variables. This is parsed, and two key variables are used; locations of destinations, and time spent at the airport by users travelling to these places. This is then normalised and used to create surface that can inhabit space, translated in a similar way to the first experiment. Similar to a 3D graph, with units on each of the axis', the form becomes unique to Wellington, providing areas of density and sparsity (fig. 3.43). This form can then be used in various locations such as walls, ceilings and bathroom benches.

6 Data is publicly available from http://www.flysfo.com/media/customer-survey-data

1 INPUT

Data is assembeled into a

spreadsheet from online sources

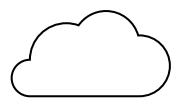

2 PARSING

Grasshopper reads data and

creates lists for time spent at airport

$v$ destination.

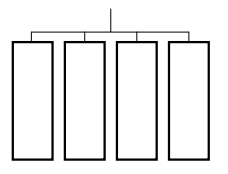

3 TIME TRANSLATION

For each destinaiton a profile is

created, similar to a graph of time

$\checkmark$ amount of people.

4 LOCATION TRANSLATION

Each profile is distrubed along a

contiuum depending on the distance

from the ariport to the desitination

5 FORM CREATION

Profiles are connected to create

forms.

3.40 Method for translation for Physical Form 2. 


\section{WELLIGTON AIRPORT SURVEY DATA}

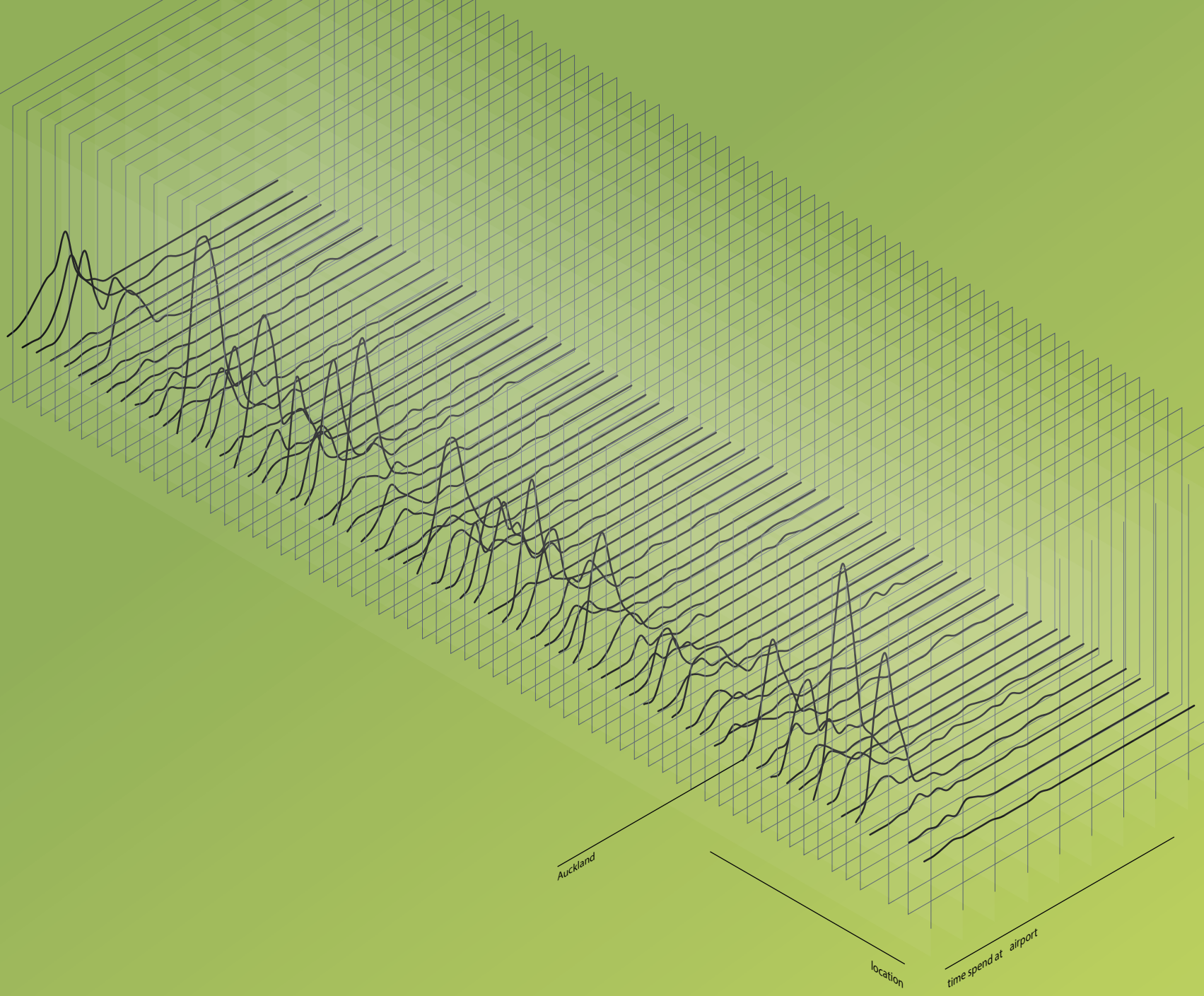

3.41 3D graph of data between duration in the airport, destination and number of people. 


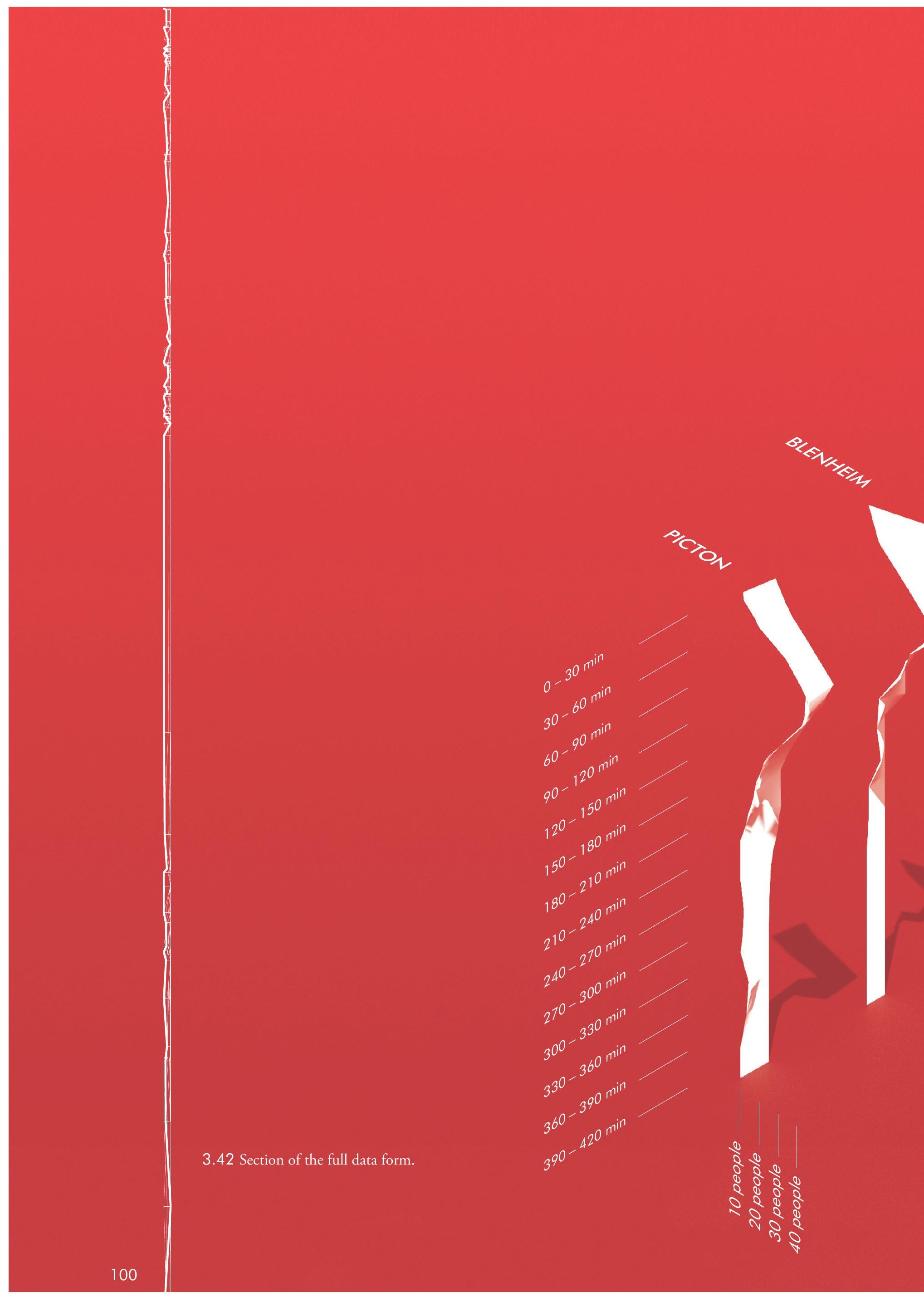




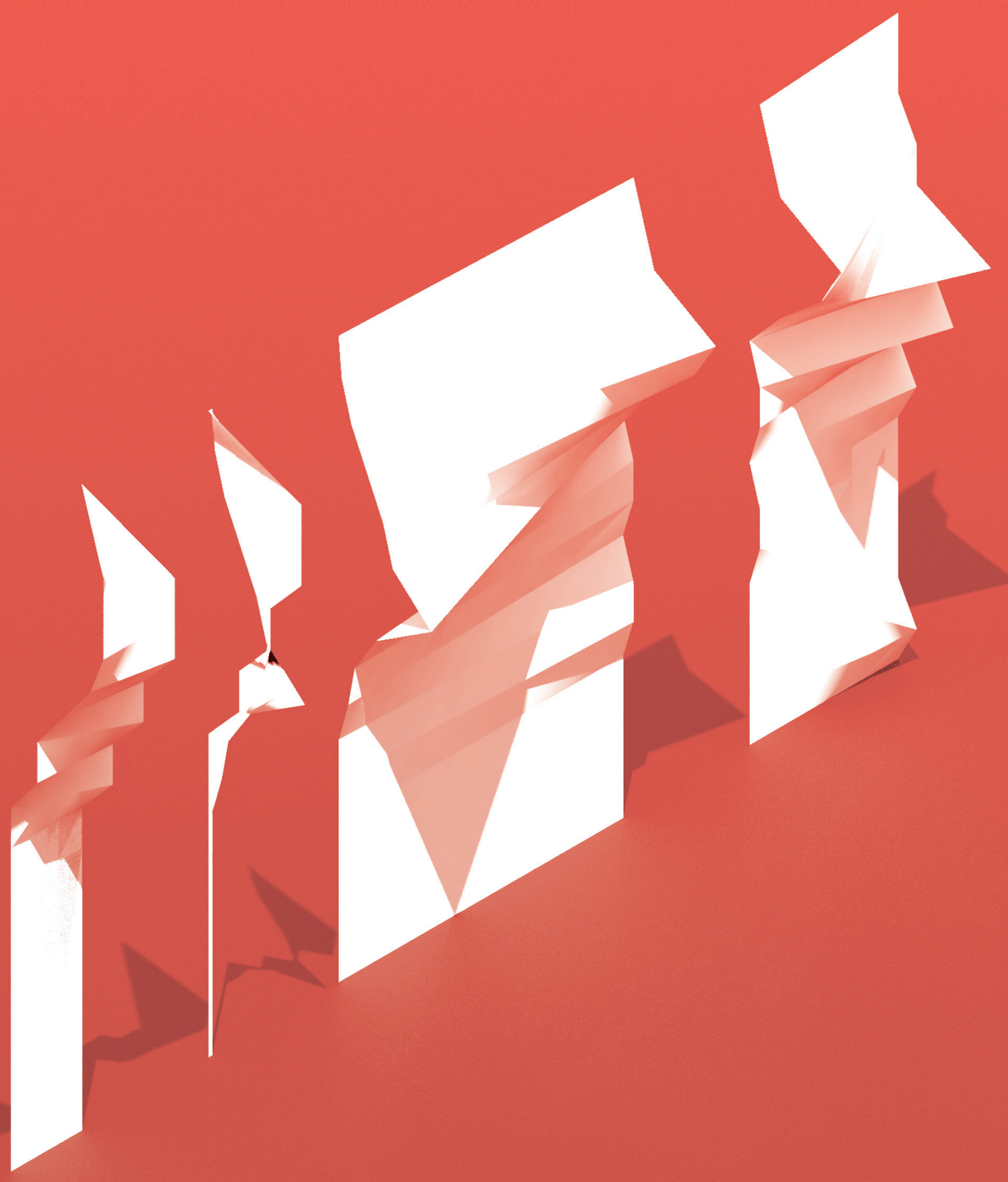


3.44 CNC routed pine form of Visitor Survey Data Set. 

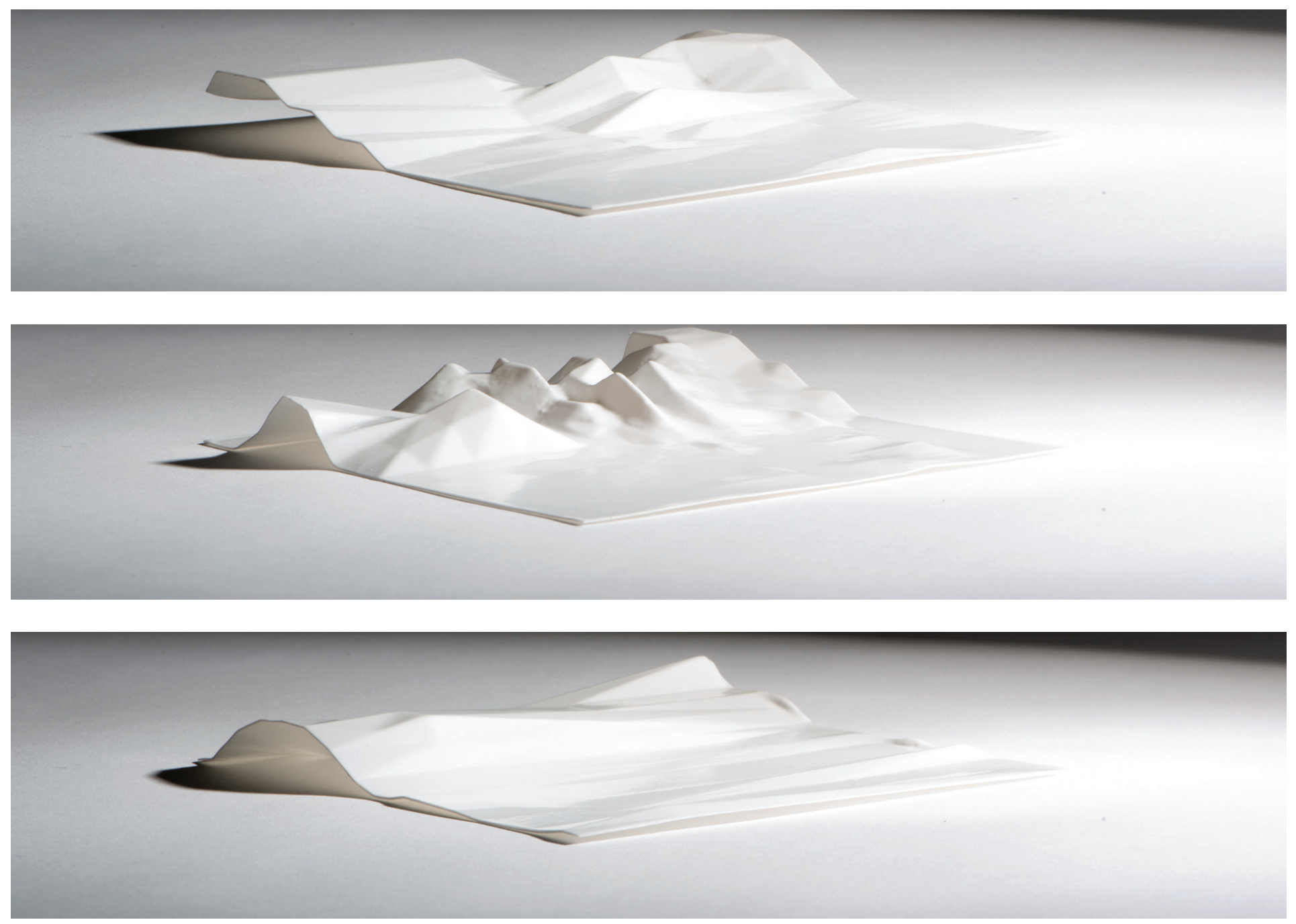

3.45 Vacuum moulded ABS over $\mathrm{CNC}$ routed form. 

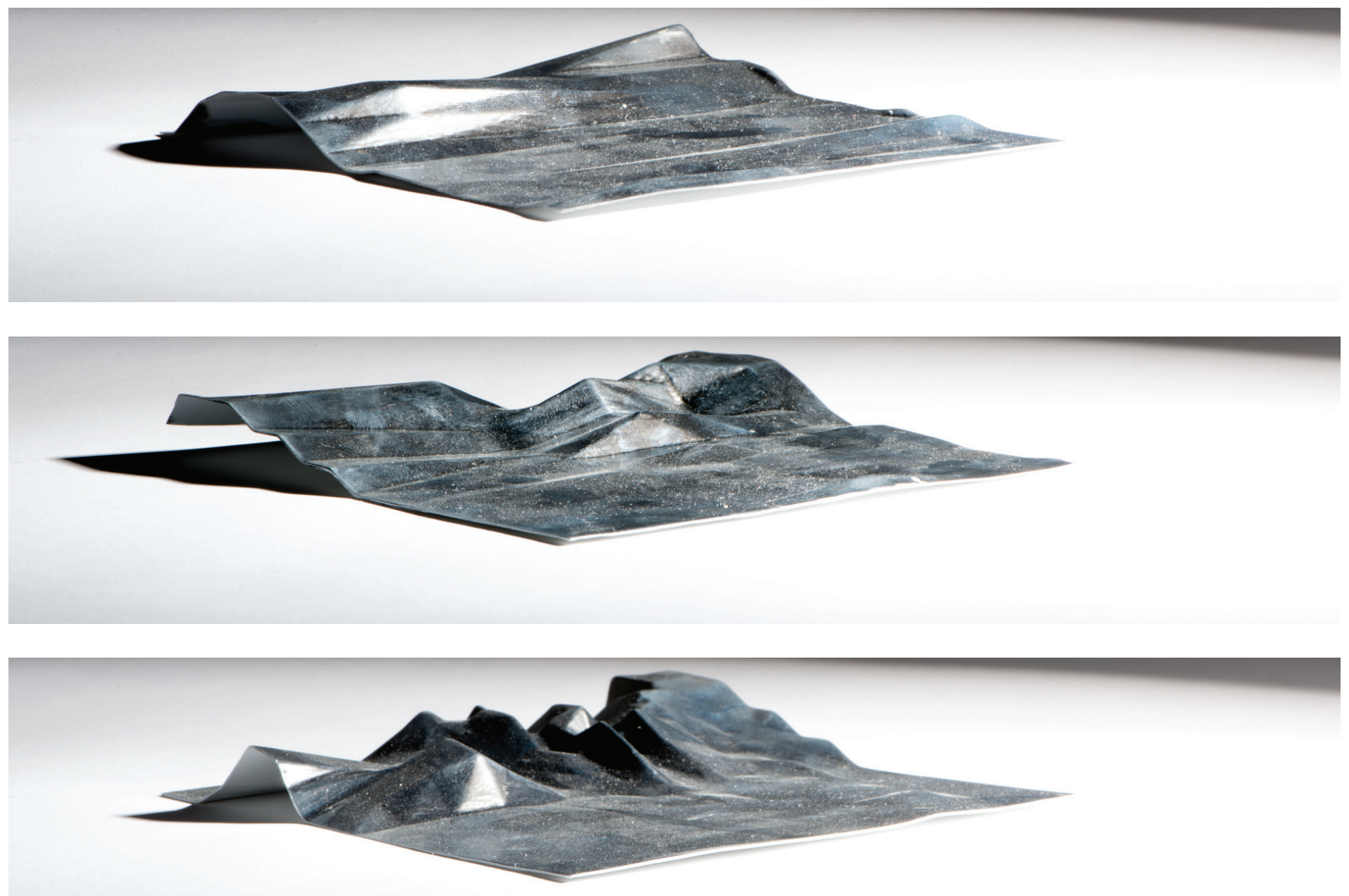

3.46 Vacuum metalised vacuum moulded ABS over $\mathrm{CNC}$ routed form. 

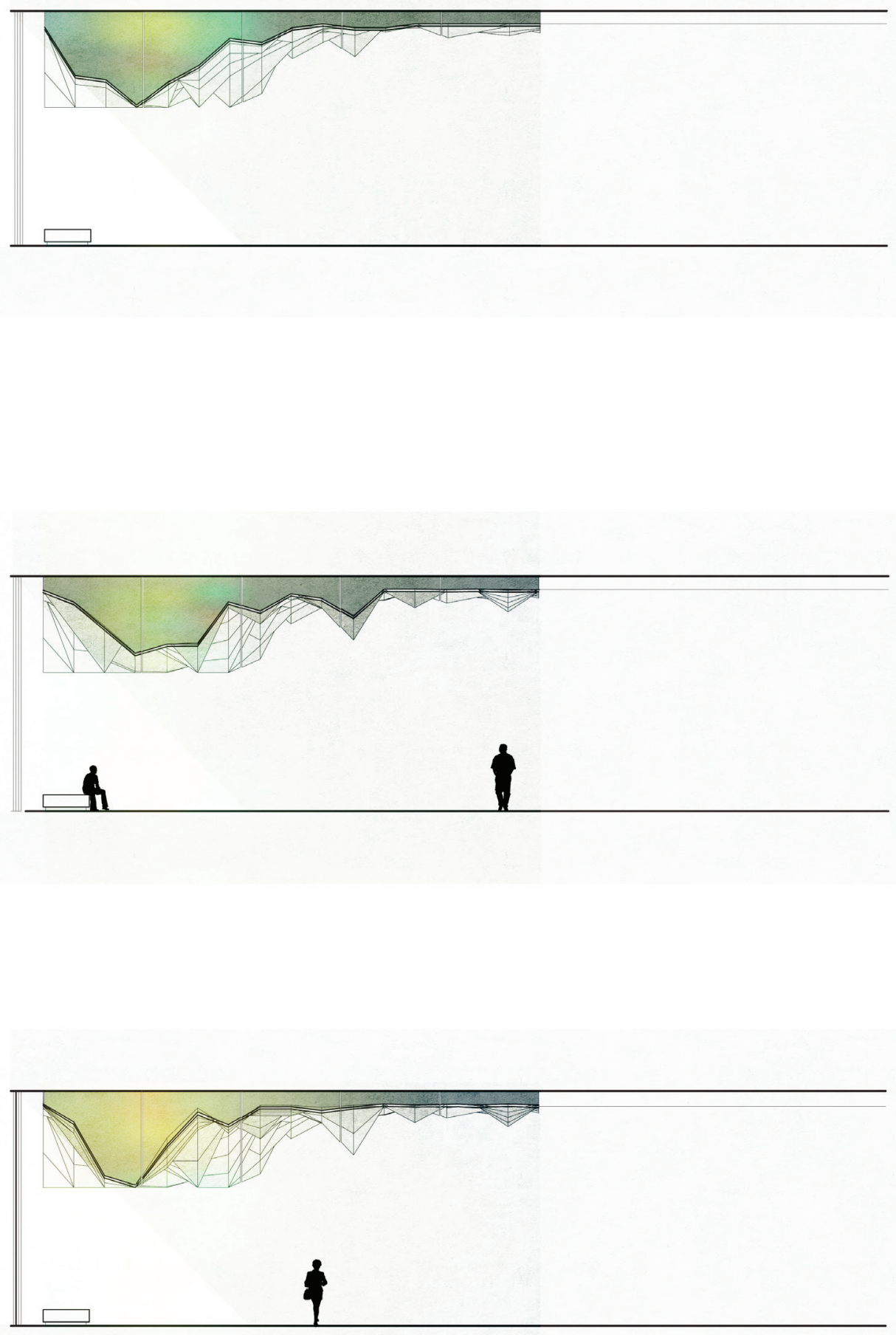

3.47 Latitudinal sections though an airport space with a density towards the seating area, encouraging congregation and socialising, done through data translation. 


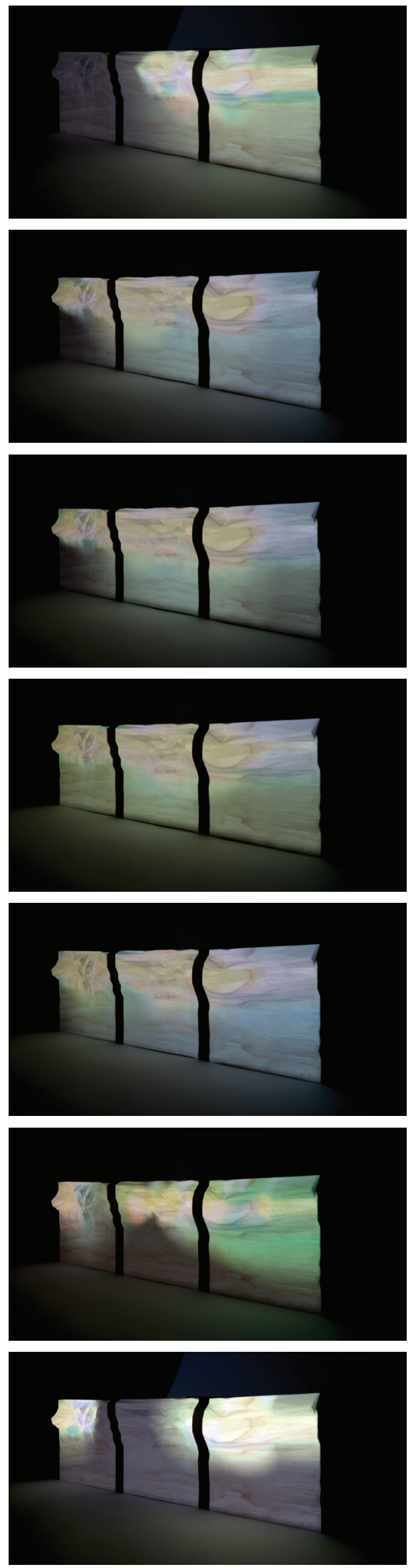

$\varangle 3.49$ Time Lapse of projections on CNC forms taken at 2 min intervals.

$\rightarrow 3.50$ Reflections from metalised forms illuminating the surrounding space. 




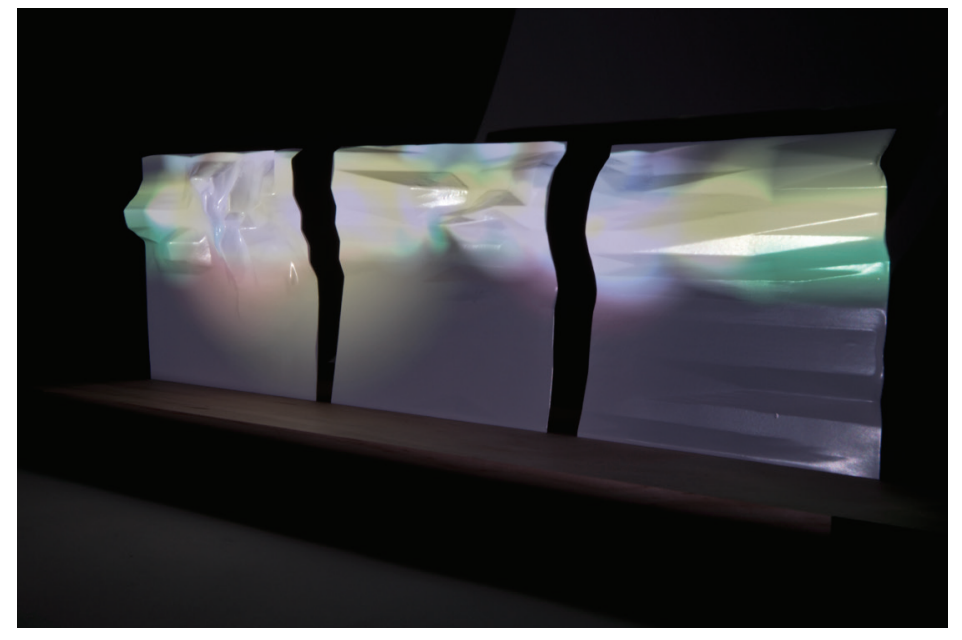

$\longleftarrow 3.51$ Luminescent forms signalling a built in screen interface.

$\uparrow 3.52$ ABS projections. The projections congregate at the density of data points. 

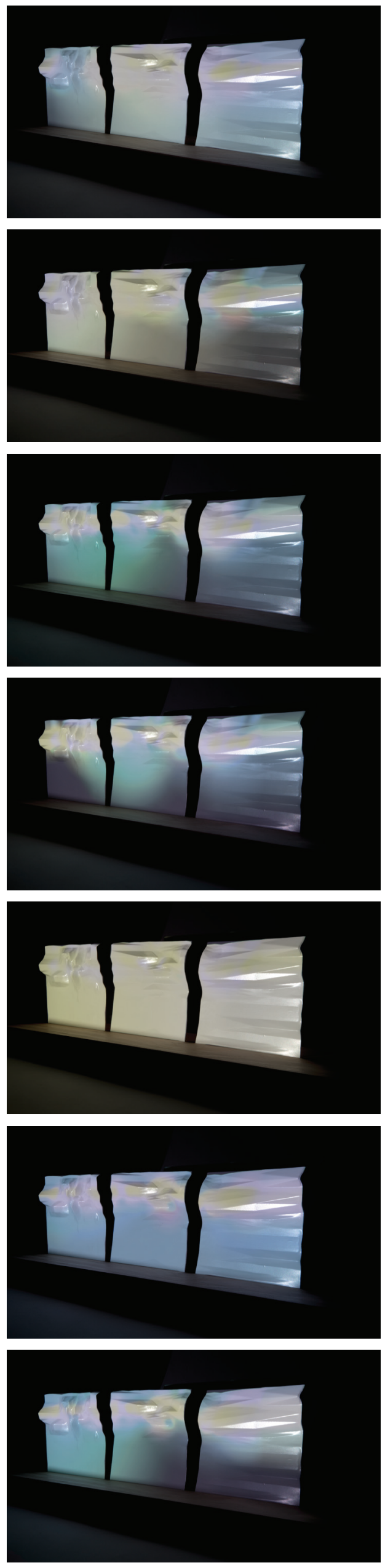

3.53 Time Lapse of projections on vacuum moulded forms taken at 2 min intervals. 

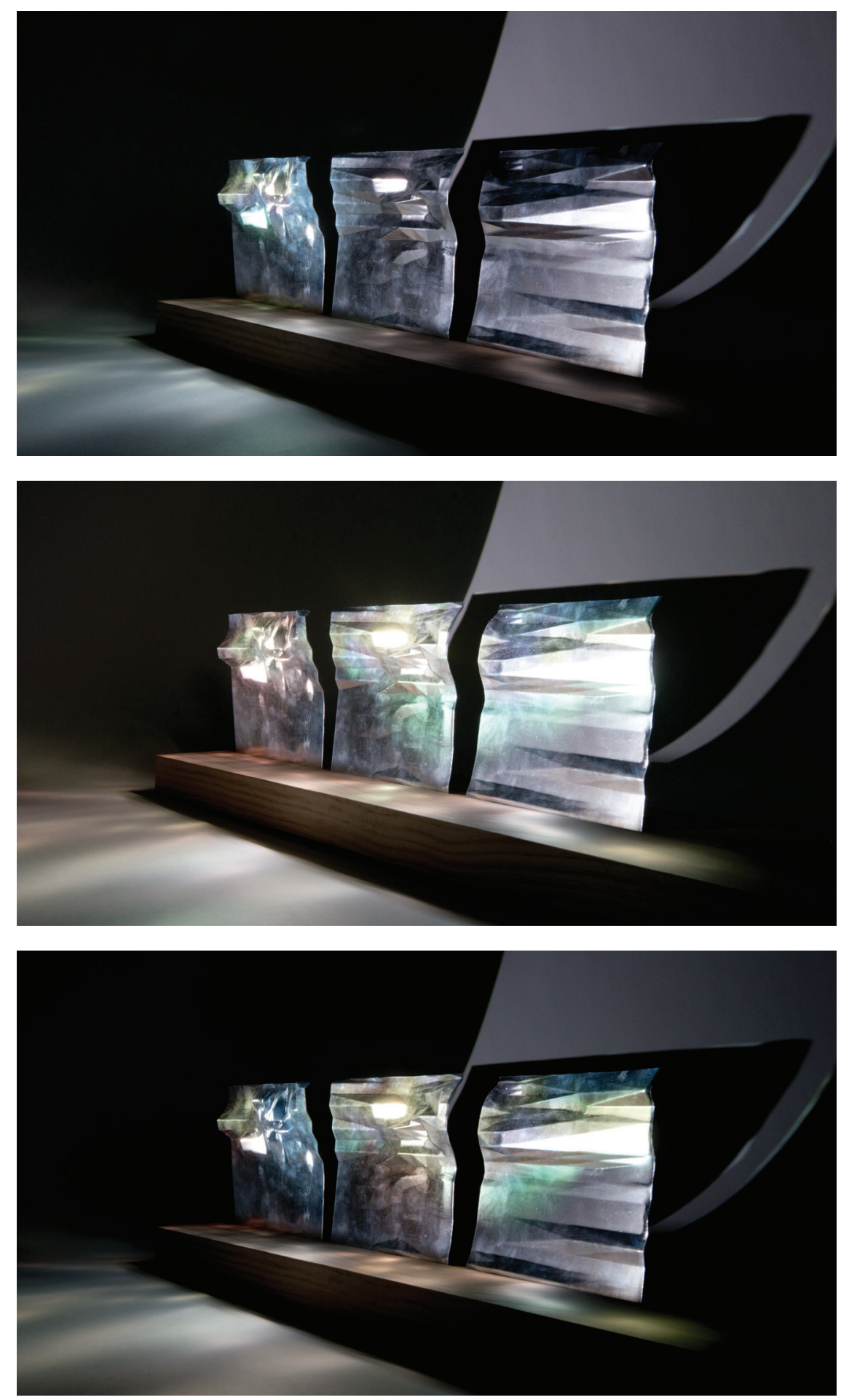

3.54 Time Lapse of projections on vacuum metalised forms taken at 2 min intervals.

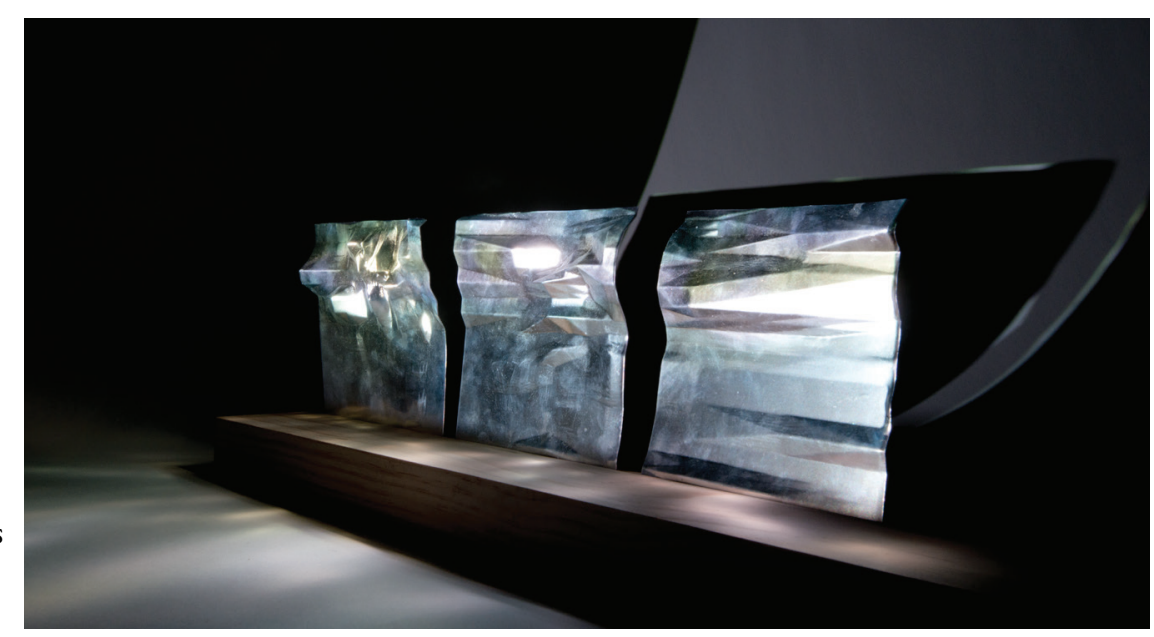




\subsubsection{ORGANIC USER INTERFACES}

Data is displayed onto the environment through non regular screens. Vertegaal and Poupyrev (2008) overview a future where 'we will use digital appliances where the space of the computing device itself becomes one of the key variables of interactivity.' For this project, in the case of 'digital appliances', architectural installations are used. The deformation of surfaces cause interactivity, both input and output of the system, with the merging of the physical form and the display into a tactile experience, "user interfaces with non-planar surfaces that actively or passively change shape via analogue physical inputs."

The seamless interaction with the connected object informs the form and way the displayed data is viewed. It is displayed through both screens and form. Input of location awareness from wearable technology to the spatial computation defines the way the installation is perceived. At an architectural scale, ONL Architects' Salt Water Pavilion contains a real time Human Computer interaction method, physical movement in space alters environmental conditions, such as sound, light and form. This thesis project uses light on form, however could be extended to involve altering shapes, further combining and enhancing the mixed reality installation. Through further exploration, the development of a robotic surface could be beneficial, although it is against the decisions made at the end of Chapter 2 with kinetic surfaces being classed as too difficult.

\subsubsection{PROJECTION MAPPING}

Using projections mapping software, MadMapper, the earlier described projection is warped onto the surface.

These projections are warped to fit to the human data centric 'sculptural forms' to allow more about both the projection and from to be learnt. Understanding is increased by knowing the attractor points the projection will move towards. This involves understanding the unfolded net of the physical form. It's this virtual physical link that creates a cybrid architecture.

\subsubsection{CYBRID THEORY}

Designing Mixed Reality (2007) outlines cybrids, integrated inhabits of mixed reality which form a physical/virtual

"IMAGES ARE PEELED AWAY FROM WALLS, CALIBRATED TO MATCH THE DEPTH AND DETAILS OF ARCHITECTURAL FACADES, AND, MOST RECENTLY, USED TO ENERGIZE SCULPTURAL FORMS."

\section{- CREATIVE APPLICATONS.NET} object. It could be considered the developed system is a form of cybrid, with physical hardware and a digital virtual display, similar to Hyzolic Ground. Projection mapping digital material onto physical surface forms augmented reality through iterations, adding detail and intelligence.

The various technologies and mediums allow the project to inhabit multiple locations of the virtual real continuum, engaging the human audience whether through personal devices, in social situations or while circulating the space. Forms raise concerns about the use of data; what types should be immortalised, and what should be used as real time projections. This led to data sets of published data, so that, in the same way architecture is of the time it is designed and built, so in the data driven $3 \mathrm{D}$ form. 
The ceiling projection is augmented to form allowing 'a user to work in a real world environment while visually receiving additional computer generated or modelled information to support the task at hand' (McCullough, 2004). Adding virtual elements to the physical allows equal experiences by all in the space without special equipment or software (Anders, 2007), often an issue with mixed realty designs. Hence, virtualised data about humans occupying the space digitally fixes itself onto the physical building, creating a cybrid of the activity happening within. Using Saltwater Pavilion, the inhabitation of data intersects users' senses to enhance environmental and social awareness in space through technology.
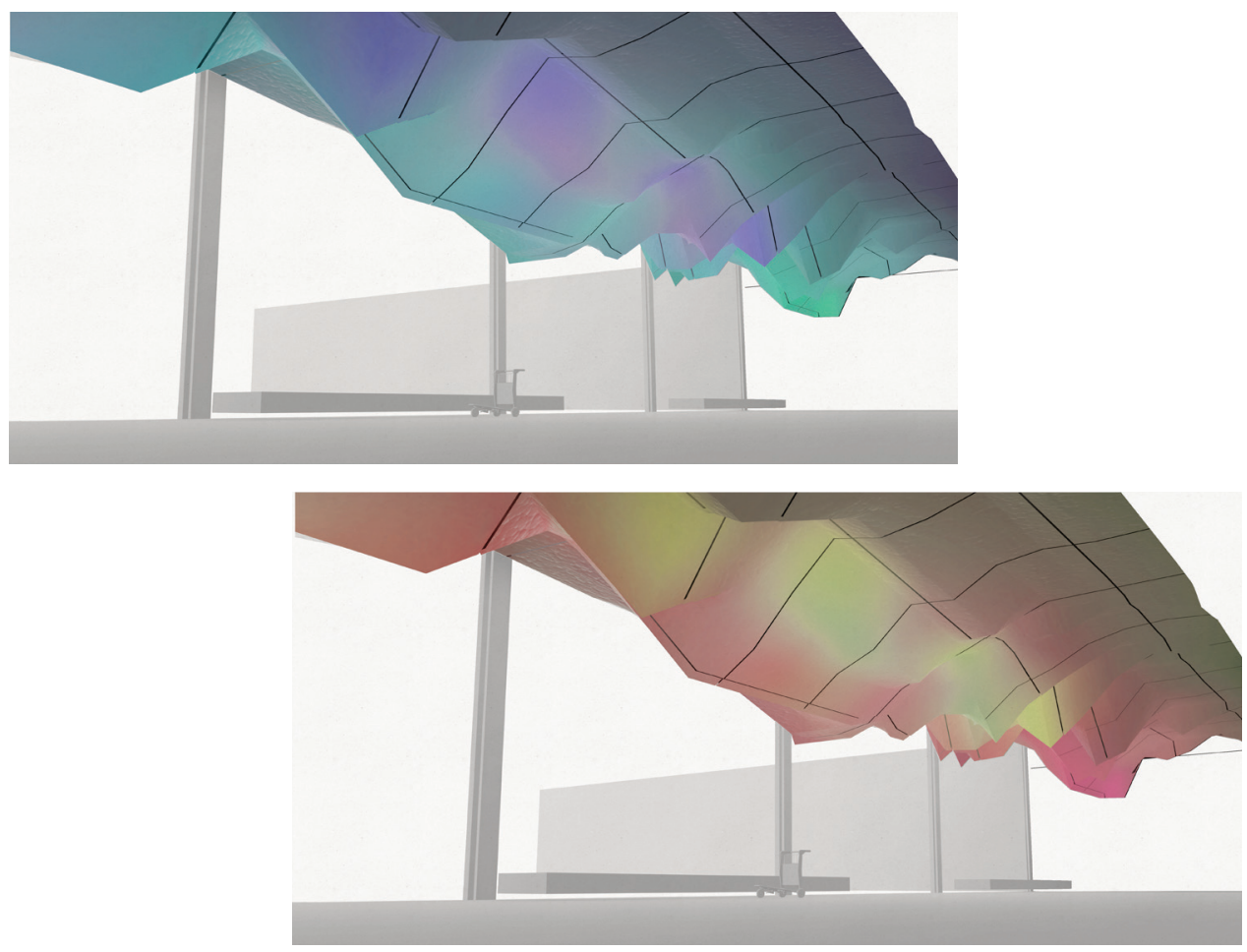

3.55 Rendered projected visualisation signalling change in hue. 


\subsubsection{LIGHT AS MATERIAL - MAKING THE VIRTUAL PHYSICAL}

Projecting on a surface, digital 2D media is 'calibrated to match the depth and details' to become part of the forms and facades, linking the mediums (CreativeApplications. net, 2014). Physical form is installed in space, becoming floors, ceilings and facade lighting, to create a constant experience throughout the space. The physical surface's material perception changes depending on the way it is projected on, for example, shadows cast across the surface creates areas of light and dark. Alteration of surface qualities in real time provides a virtual mapping of the physical worlds, conveying 'past, present or future information about a place or object'. The physical surface reflects light around space depending on material qualities and what is being projected. This displays corroboration between realties with intrinsic connection between form and projection, ensuring cohesive design. Salt Water Pavilion is referenced, with a form that is reminiscent of the movement of users which flows across the surface. The elongated form then allows projections and light to accentuate this movement.

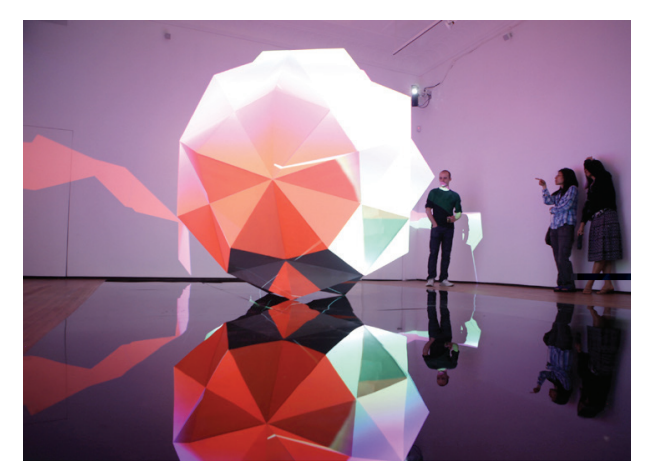

3.56 Parmenides, by Dev Harlan (2011), projection mapping on wood, foam and plaster object.

The series of form experiments transform human-centric data into a $3 \mathrm{D}$ form. Data in constant flux is not used, as it is dynamic, instead data of consistency or a meaningful point in time is chosen. Two experiments take place with two sets of data, using a mapping of values to coordinates in a 3D grid (Wu \& Clayton, 2015). Projections then respond to form through mapping and attractor point locations.

This project works to meet the performance index. Physical and digital elements have corroboration and reciprocity through their intrinsic link to user specific data sources. This data usage also gives it an anthropic design that creates a unique and informative human computer interaction. The space acts as an extension to the usual social happenings in the space, amplifying the understanding of circulation in the space while remaining discrete. 


\subsubsection{SUMMARY}

The creation of forms to project visualisations on explores a cohesive object that is of the initial data. Forms are a layer over the existing architecture, providing an internal data skin for users to inhabit. Matching visualisations to form became difficult as the form is beneficial when it enhances the data displayed, making the case for Organic User Interfaces's to be explored for these large transport spaces. 


\subsection{HUMAN LOCATION}

The use of the actual location of humans into the designed system allows for a greater level of personalisation. This is done through three phases; the development of a smart phone application mock up (v1), a prototype (v2 \& 3) and wearable mock up.
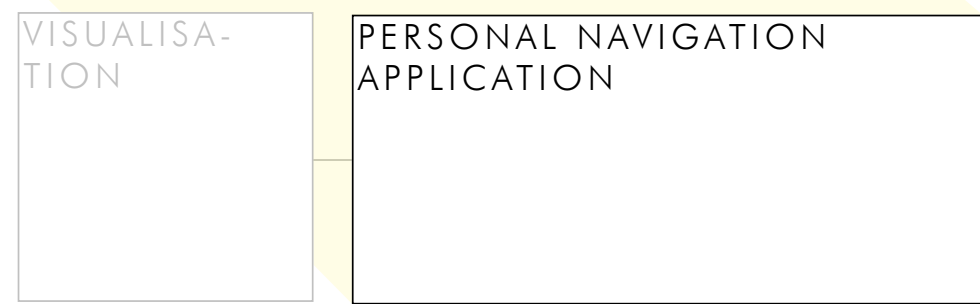

\begin{tabular}{l|l|}
$\begin{array}{l}\text { ANDROID } \\
\text { MOCK UP } \\
\text { VERSION 1 }\end{array}$ & NETWORKING \\
\begin{tabular}{l|l} 
PHONE \\
INTERFACE \\
VERSION 2\&3
\end{tabular} \\
\begin{tabular}{l|l} 
WEARABLE \\
INTERFACE
\end{tabular} \\
\hline
\end{tabular}




\subsubsection{APPLICATION DESIGN}

\begin{tabular}{|l}
\hline APP \\
VERSION 1
\end{tabular}

3.57 Working android application (V1) mock up is used as an initial design, by directly porting the programme to a touch screen device. 


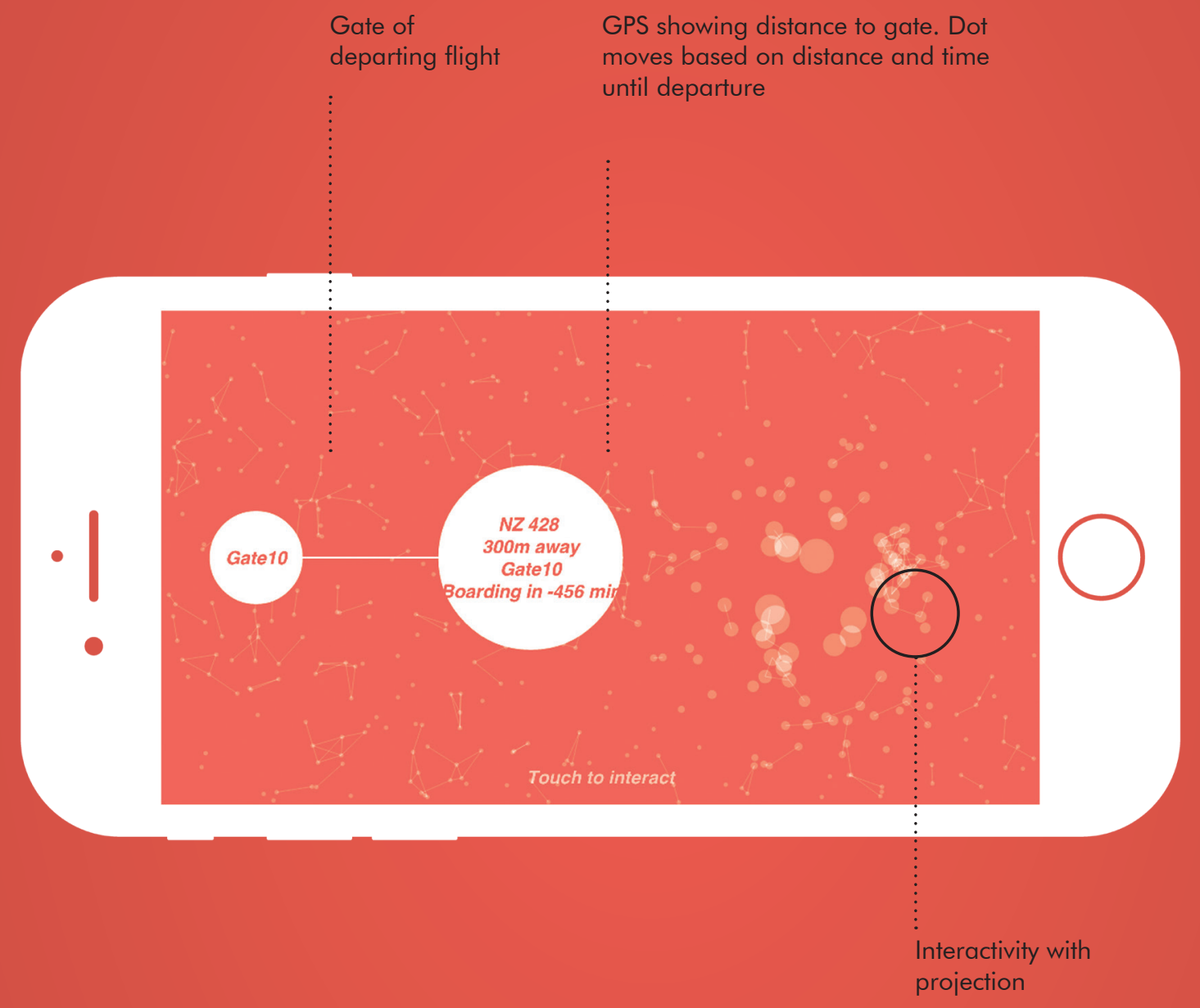

3.58 Method for application, informed by the visualisation, producing both an interface design and prototype. 


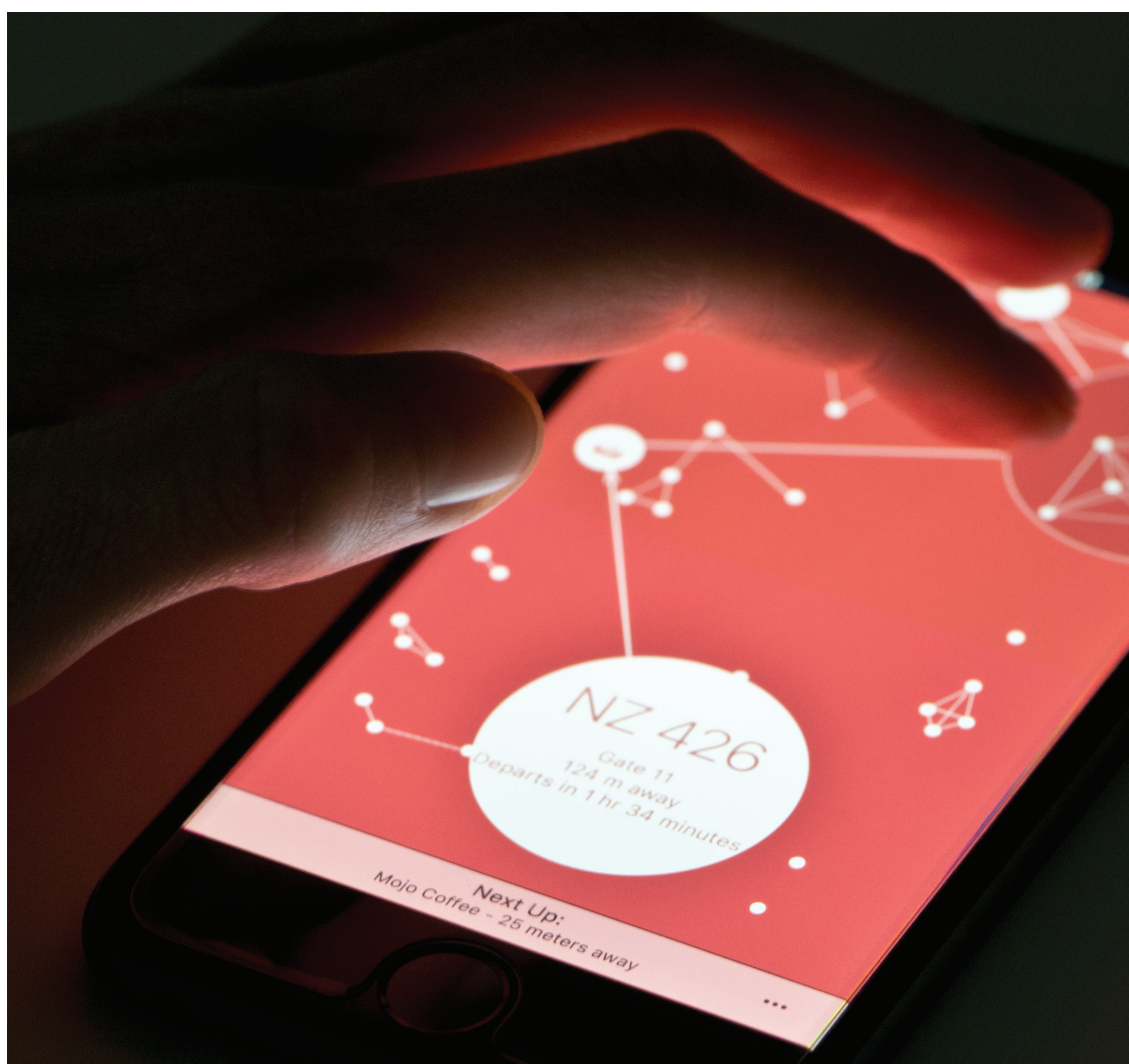




\section{APP \\ VERSION 2}

$\longleftarrow 3.59$ Glow on user linking the architecture cybrid to human users in the same space, prototype (V2). This second iteration of an app design is based on the technical android mock up, but focuses on visual design and human interface. It visually communicates pathways through space.

$\checkmark \quad 3.60$ Flight selection screen (V2)

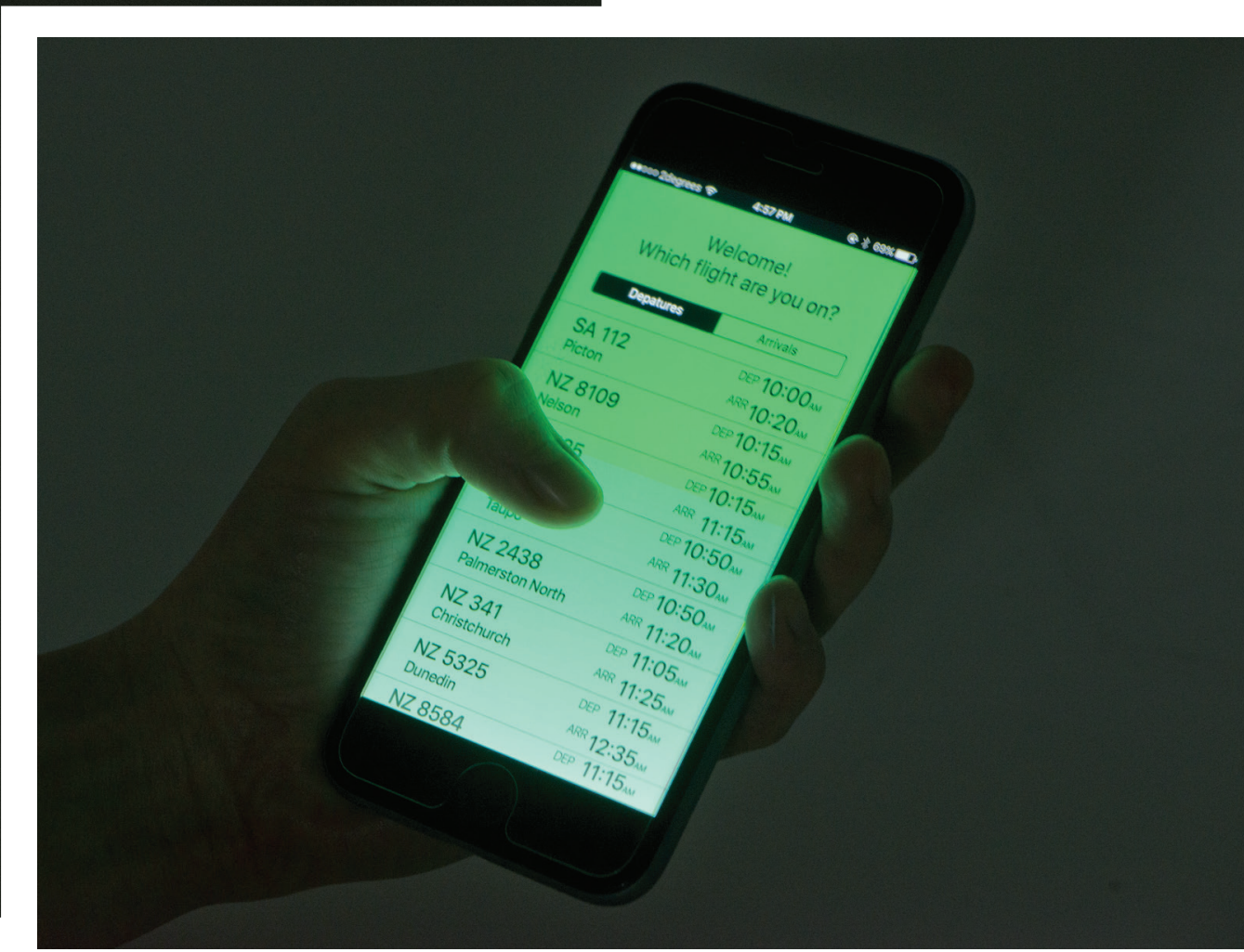




\section{APP \\ VERSION 3}
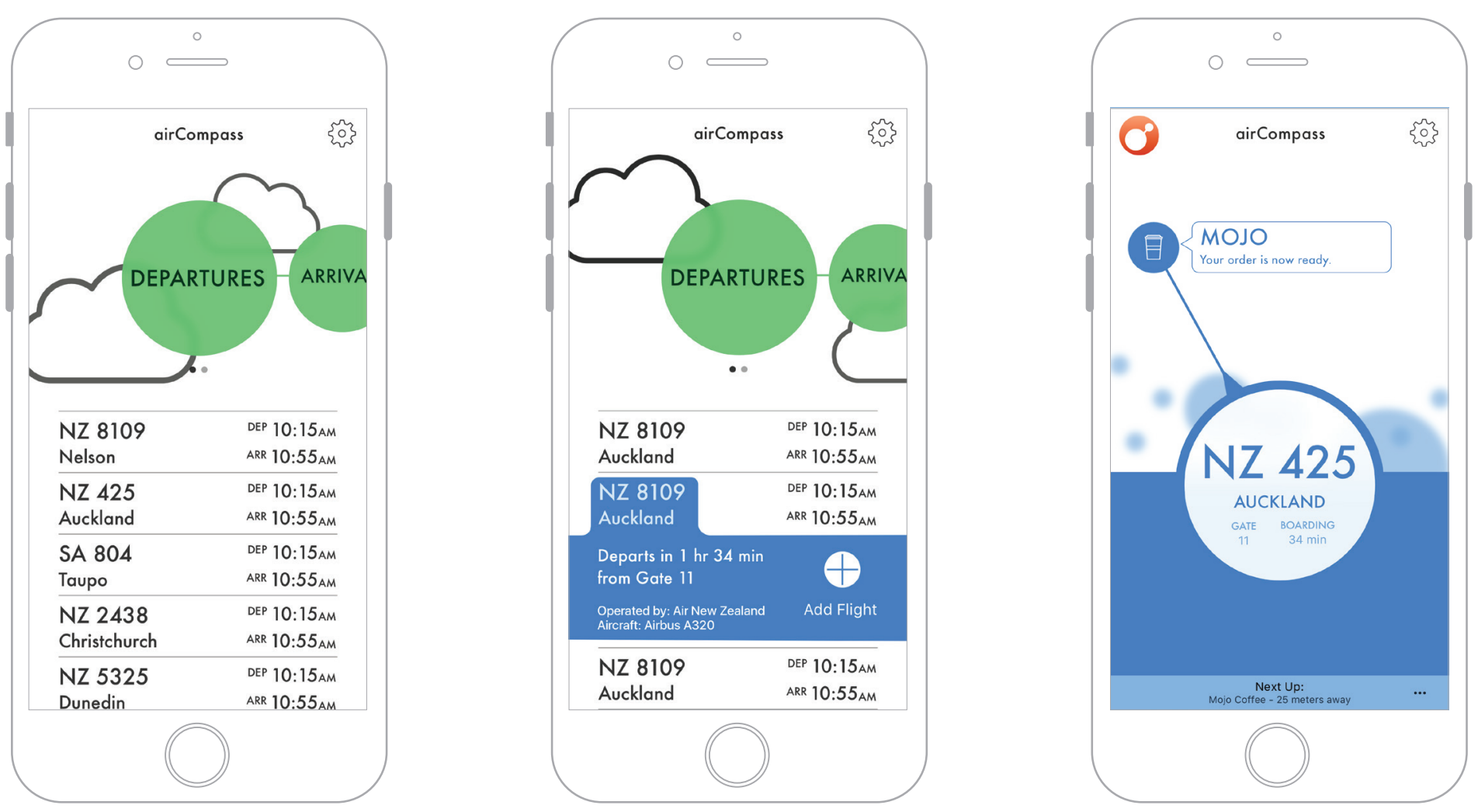

3.61 The third and final smartphone interface iteration with flight selection, navigation pane and itinerary options. Navigation uses specific architecture points as an interface. 

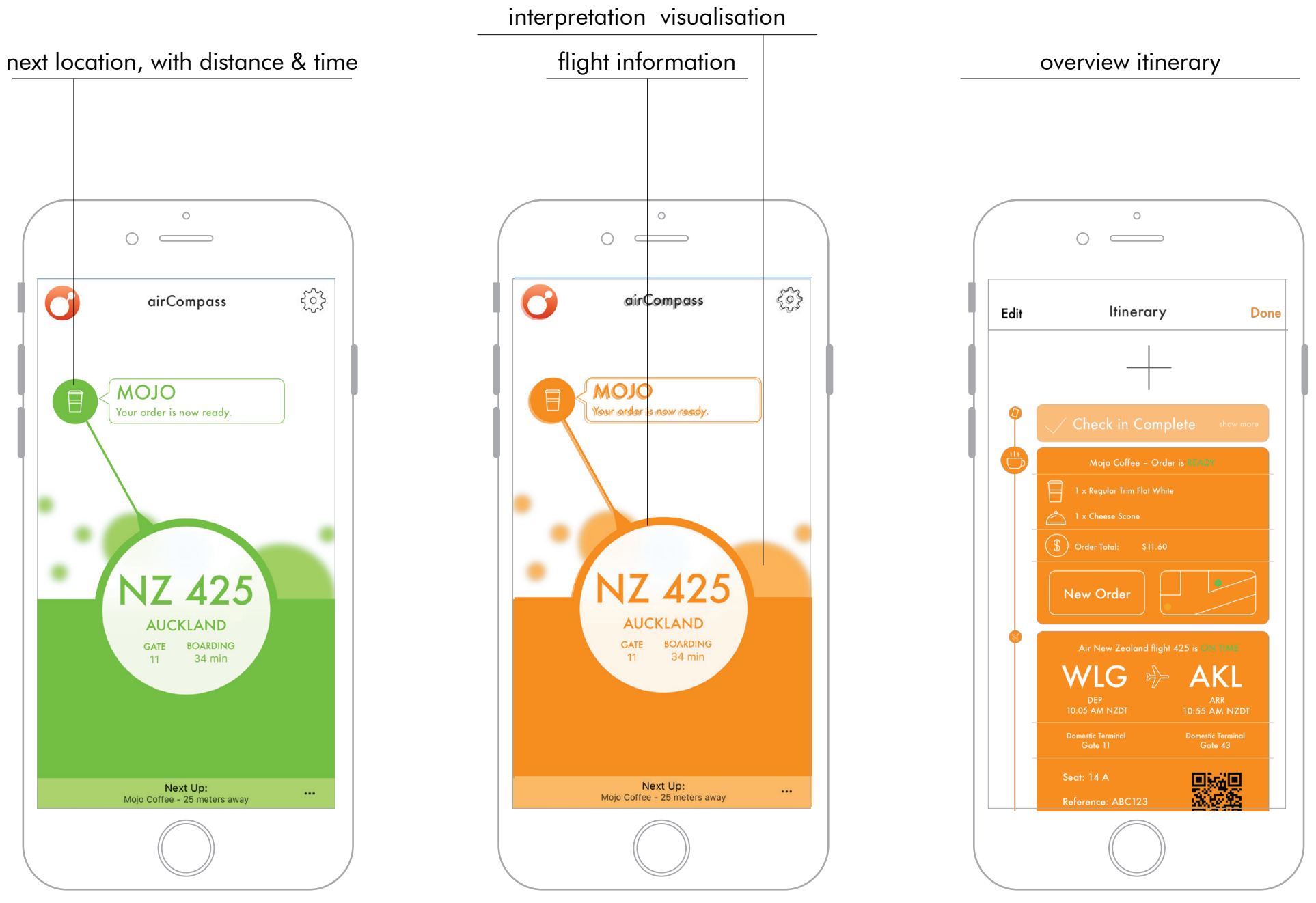

THE SMARTPHONE APPLICATION ALLOWS USERS TO SIMUTANIOUSLY ENGAGE VIRTUAL AIRPORT SERVICES AND THE PHYSICAL AIRPORT SPACE. 

All the current arriving and departing flights are displayed on opening.

Selection of flight.

Live display showing other people on the same flight, and directions to the next destination.

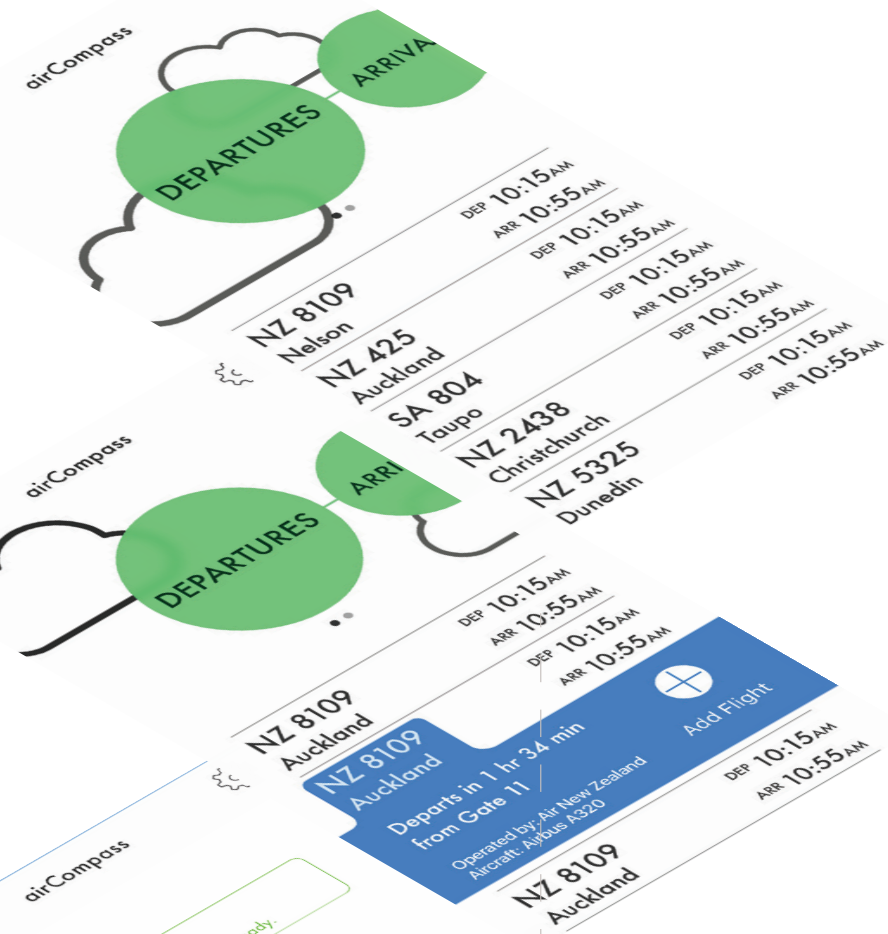

Personal Itinerary helps navigate the airport and keep to time.
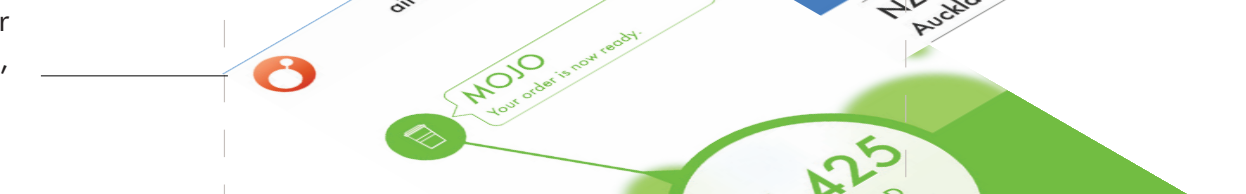

3.62 Navigation through the interface on smartphone and link of phone graphic to the spatial visualisation. 


\section{WEARABLE}

APP
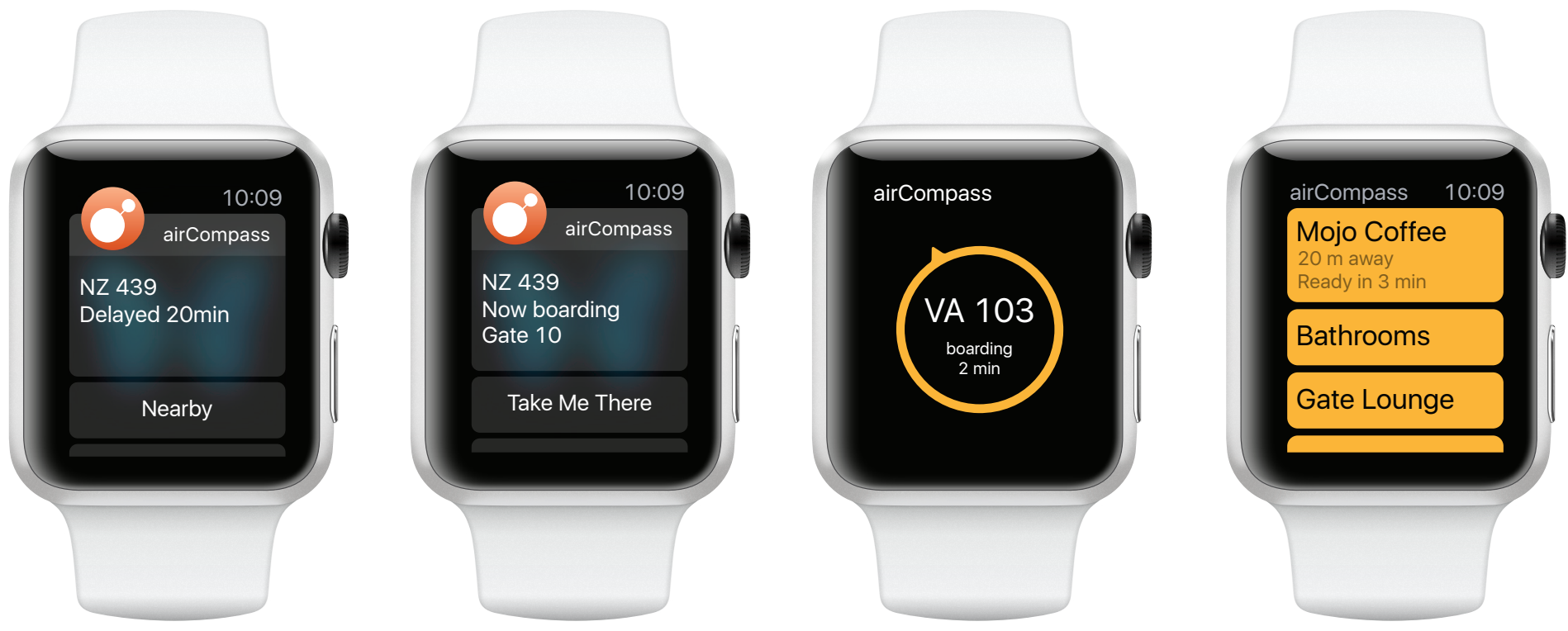

3.63 Wearable application to use on-body technology to transmit location to the spatial system. It also provides an interface and sensorial feed back to the passenger. 


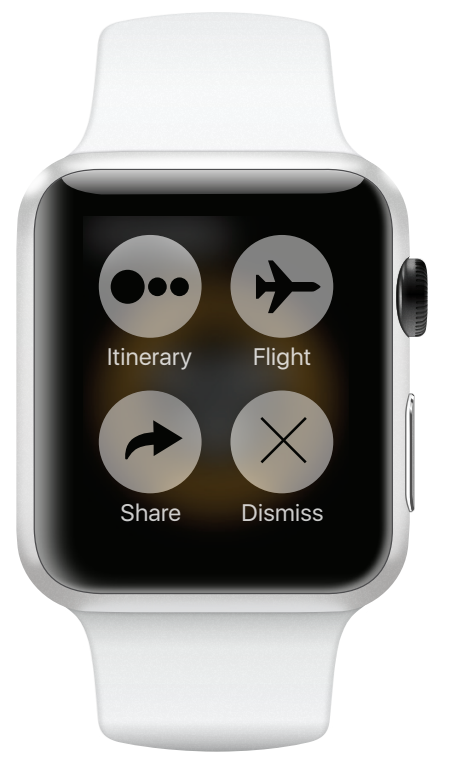

\section{VIRTUAL COMPASS}

The system acts as a compass with the space. In the same way a compass helps to navigate terrain, lit helps to navigate buildings socially. 


\subsubsection{SMART PHONE APPLICATION}

Location systems, Indoor Positioning Systems (IPS) ${ }^{7}$ and Global Positioning Systems (GPS) are integrated with wearable technology into a mobile software application, allowing a user to feed personal data anonymously into the system, and receive updates and awareness of everyone else. This use of Achten's (2015) overview of ubiquitous and developed technologies allows interactive space to become more sophisticated, helping users navigate and time manage better.

Using GPS, a prototype is built based off the spatial simulation programme (fig. 3.57). This prototype is working on an android smartphone and relays locations to a server which creates a real time representation of the users to be projected onto space. Using the conceptual framework of Unnumbered Sparks, applications can become a connected spatial controller. Based on real time location, the application visually changes, similar to the augmented reality shown in The Architecture of Radio (figure 3.64), giving users a 'passenger flow finder' to use.

IPS integration is yet to be prototyped, however using Bluetooth LE beacons ("Estimote," n.d.) would allow for an accurate representation of location in space. Projected, interpreted and individualised data helps users understand the space and its relationship to users. Users can get awareness of context within the space, see nearby people on the same flight and understand directionality to gates.

The smartphone application acts

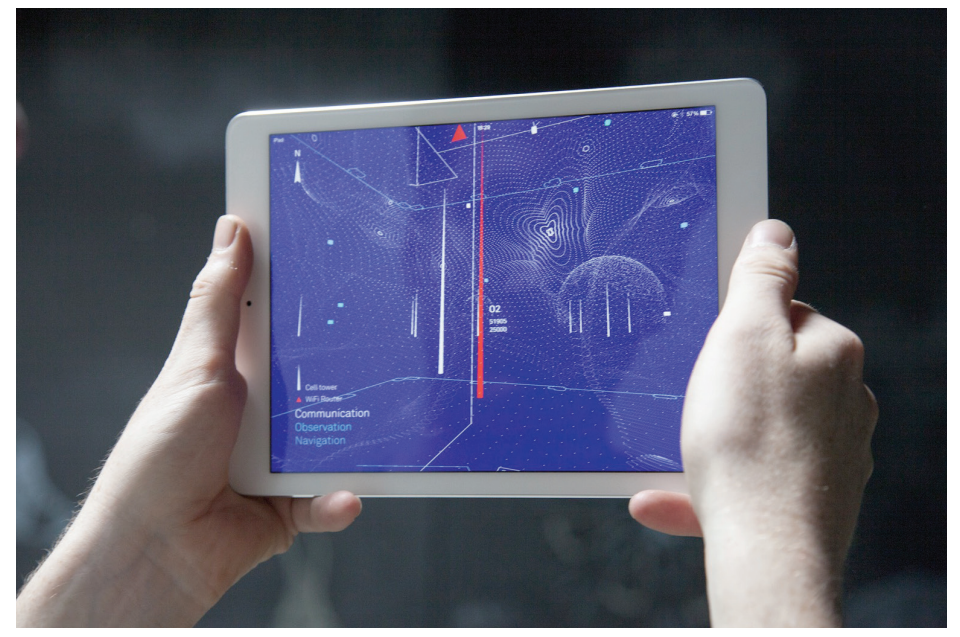

3.64 The Architecture of Radio, by Richard Vijgen, is an augmented reality viewfinder for network signals. The visible space is hidden and in the invisible shown. as an integrated input device for the spatial system along with being a personal specialised navigation device, allowing options such as programming itineraries and learn more about the space. Although it works separate from the overall system, it is intrinsically connected through an inherently similar graphic language and data types. As a personal gateway, the application pulls the location of other people in the space from the same flight (fig. 3.62). This connection informs social connections, both digitally through the device and physically by seeing screens of others. The itinerary helps users plan the use of space, and could self-update to gain knowledge about the amount of time needed to do certain activates and reach their flight on time. For example, if there were many users getting coffee at one time, the application could reorder the itinerary to decrease wait times. The space could also be optimised for the levels of users, to get accurate

$7 \quad$ IPS technology uses Bluetooth personal devices and beacons fixed to space to triangulate accurate local location. 
information to further efficiently design the space. By having multiple uses for the application, the physical and virtual experiences of space can be combined further, creating a fully integrated social experience.

Duality between personal devices and architectural space provides a set of real and virtual engagement that can be used in three ways:

- Personal device as an indoor way finding app for a building,

- Architecture as a work of installation art for space, or

- Combination as an immersive experience.

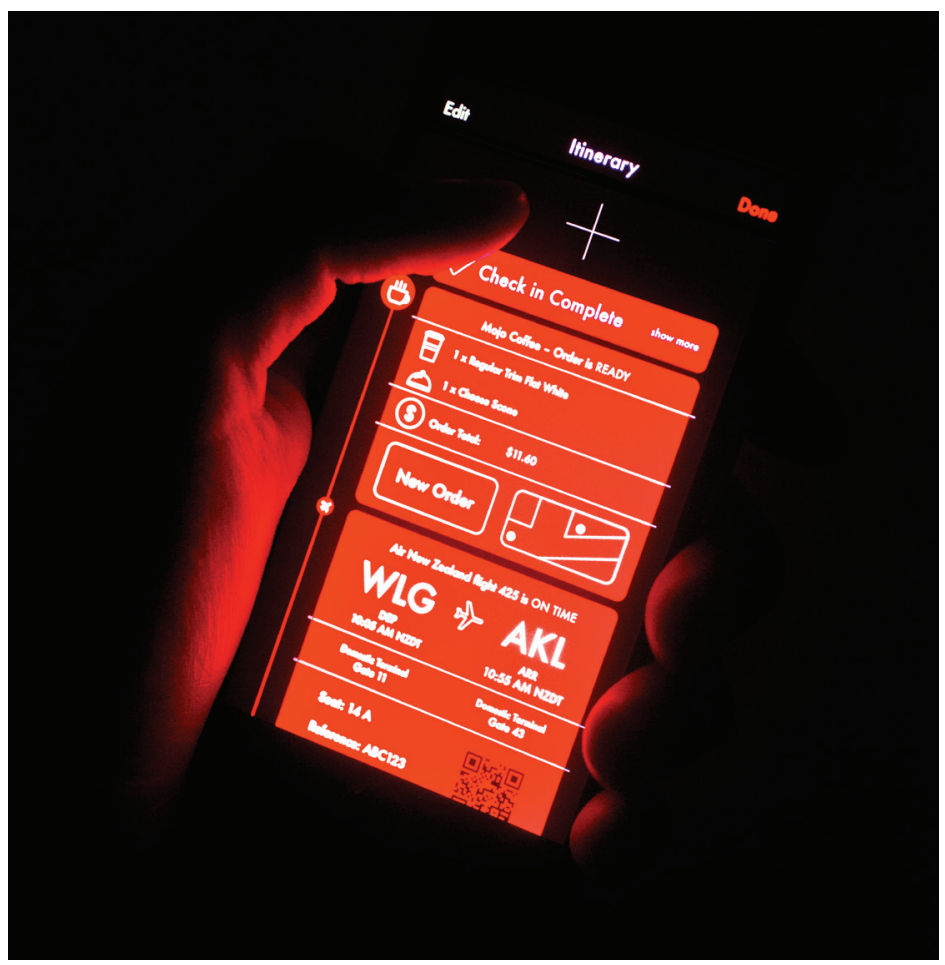

3.65 Application prototype (V3) itinerary screen projecting onto user. 


\subsubsection{LOCATIONS IN INTERACTIVE SPACE \& SOCIAL COMPUTING}

As the architecture is definitive in location, the services they contain will be also. This section aims to understand the social implications of space with precise location being given to spatial computational systems.

A Collaborative Virtual Environment is embodied through the proposed system (Segard, Moloney, \& Moleta, 2013) allowing physical communities of users to interact virtually while solving a physical problem of spatial circulation and use of space. Convergence of big and local data changes the way people use space, providing a local, custom, efficient and specific spatial experience (Achten, 1997). Location models are a representation of 'activity and organisation' of space (McCullough, 2004), the sharing of which has the ability to help those in space via a shared understanding of personal experiences. Human computer interaction is done socially, the communication is at first 'mediated by computer systems' (Dourish, 2001) but transcends into the physical. The end result is an improved physical experience than one without computation. Machine learning techniques, data mining and predictions for the use of space can change the way space is designed, which could come about through the use of a natural user interface that is passively interacted with. This machine learning can be extended and researched, allowing the system to organically learn and respond to trends of users in space (Shekhar et al., 2015).

Using Points as a design system precedent, it is important to have "just in time' information for users. For example, there is no point in having way finding to a gate that isn't boarding. There is difficulty in understanding how a similar set of data, which is perhaps more complex, could be architecturalised. The just-in-time idea is used through a logic to display the needed information, however as the information is architecture it is subtler. Extra information is given through an application.

Two points of interaction with the Reality-Virtuality Continuum allows opportunity to arise for more interaction between users. Virtual socialness is expanded into the physical world, creating easier interaction with a lower social threshold while still retaining traditional social boundaries. User tracking with provision of a virtual real time representation of realty extends social connections (Kuo et al., 2004).

The physical - virtual duality of the system uses interfaces between the two realms, made up of sensors for system inputs and displays for system outputs. Each integrates with a section of the overall system, specifically responding to part of the environment, either individually or globally. Sensor data, based around the Internet of Things are 'computational representations of physical objects' ' manipulated by virtual entities' (Anders, 2007). These connected physical objects then stream data to influence interactive space (Anders, 2007). A server/client network allows a supply of data from smartphones to an architectural client, producing projections and a smart architecture (fig. 3.66). Personal devices become another input for spatialisation. 


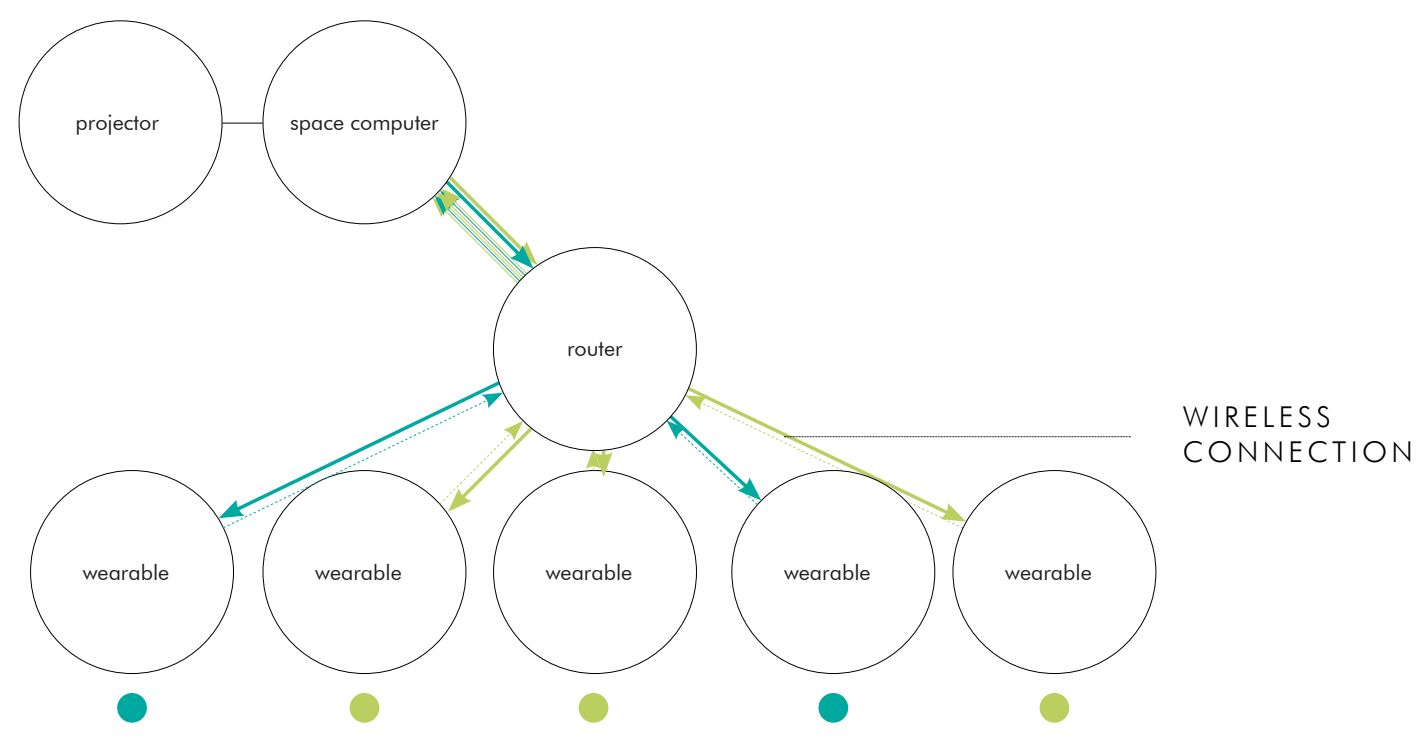

3.66 Information given by wearable and personal devices allow flight specific feedback to be given by the system.

\subsubsection{SUMMARY}

Location is an important factor in enabling navigation of space. Information is transferred through an application, feeding into the visualisation system, allowing specific points to be a true representation of people. Giving a social reciprocity between passengers through virtual mechanisms allows a crowd sourced way finding system. 


\subsection{RESULTING SYSTEM}

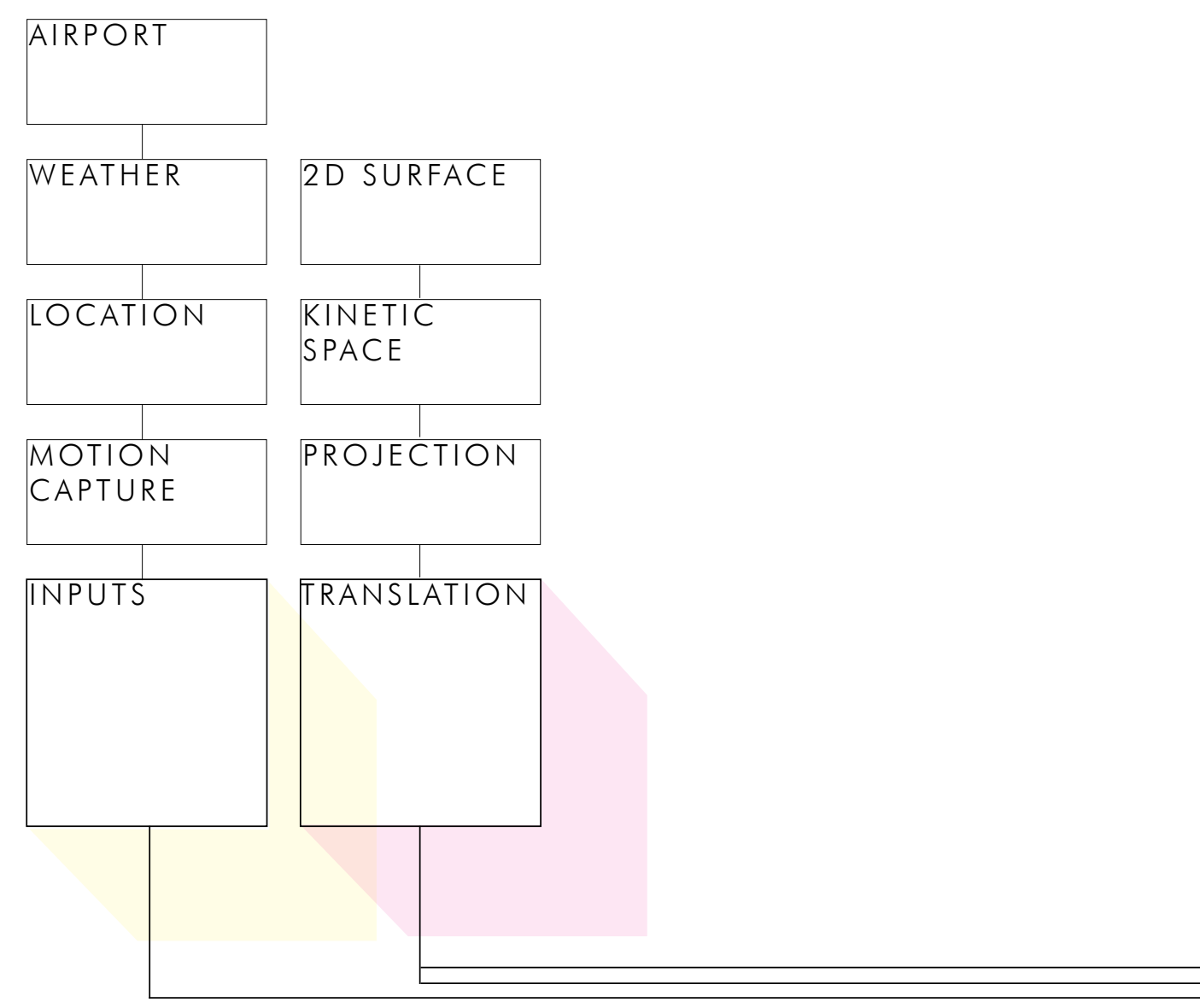

3.67 Almost all experiments which took place resulted in a compiled system. This diagram explains this. 


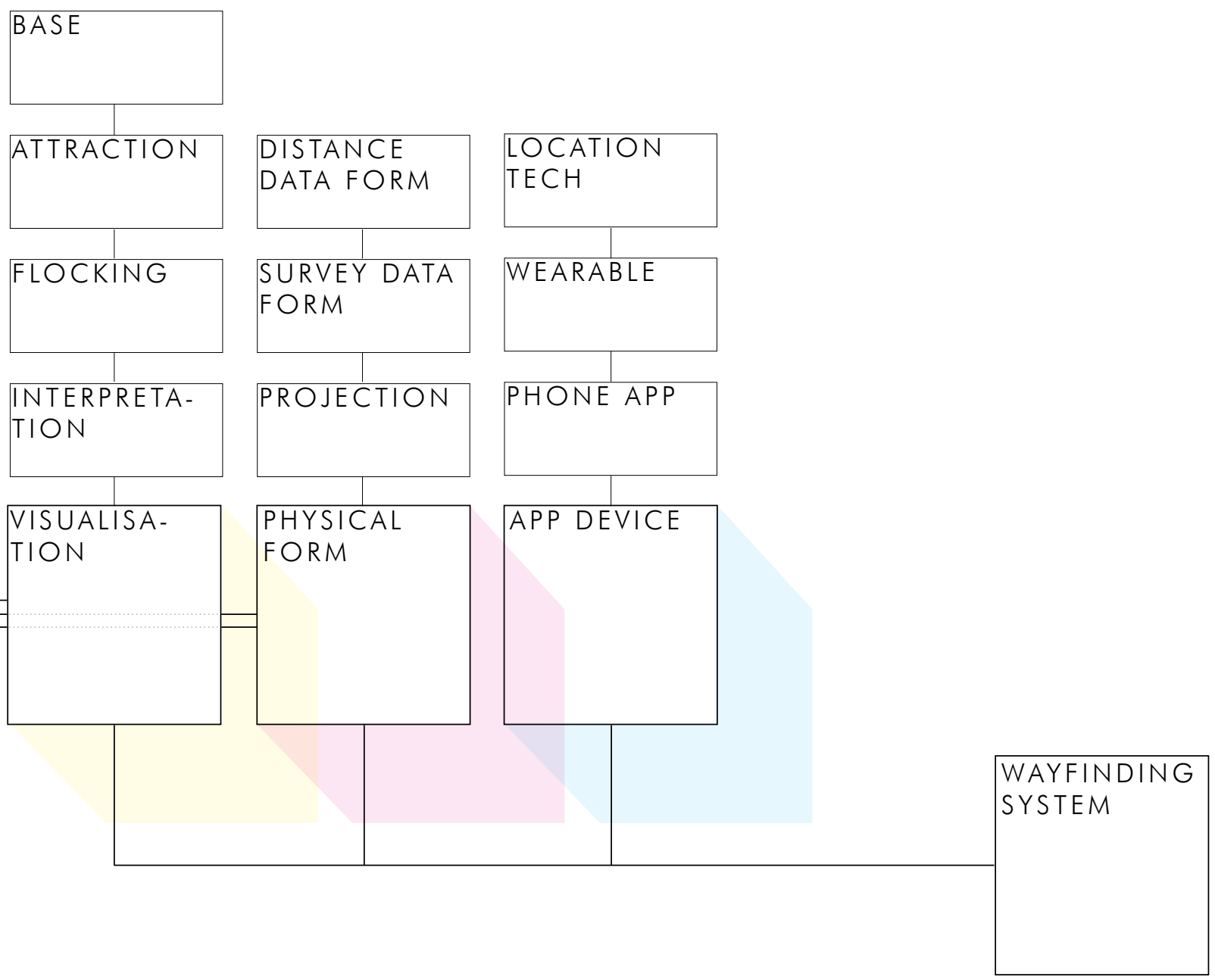




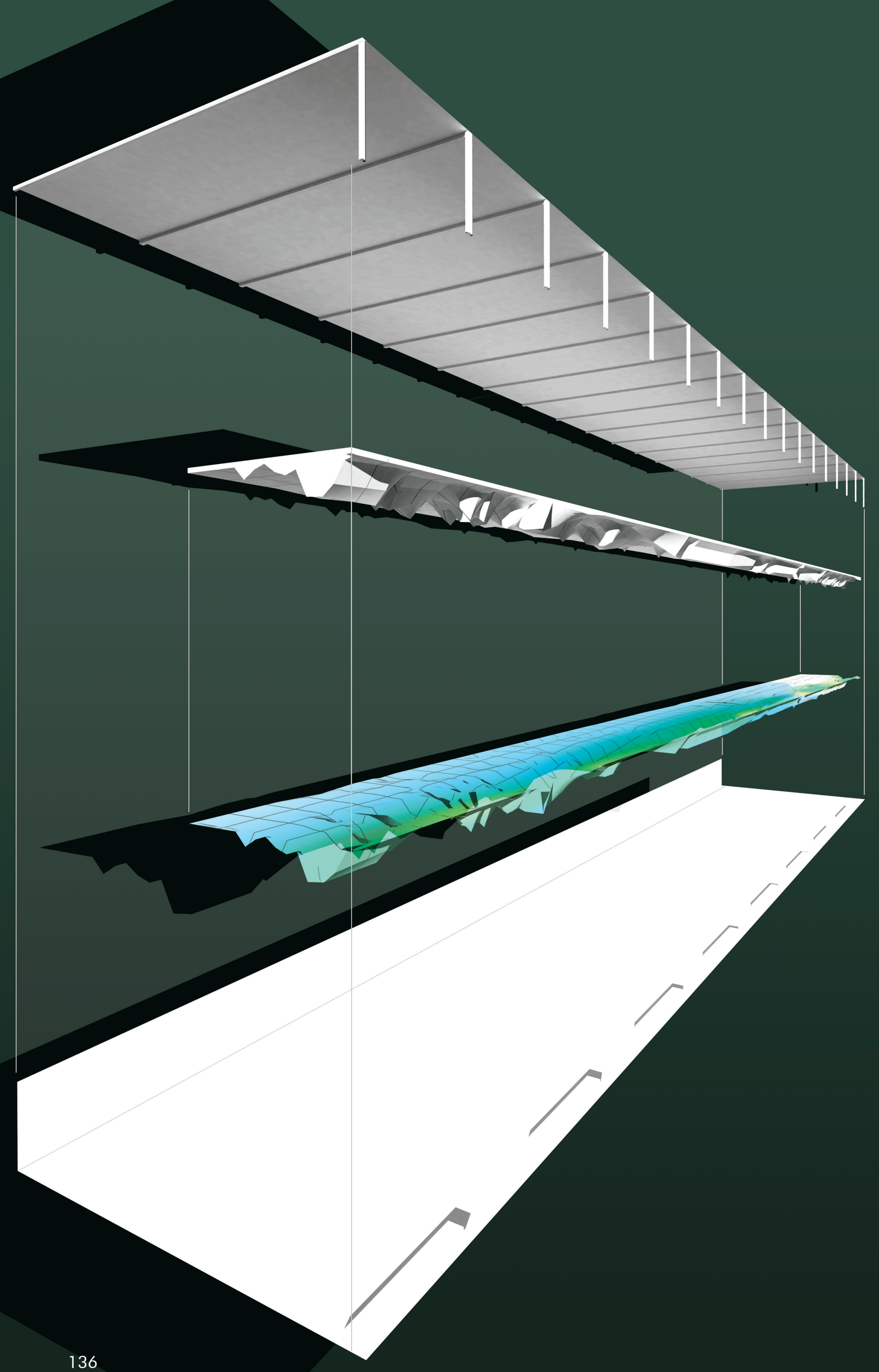



crbrid, and its location in an existing space. 




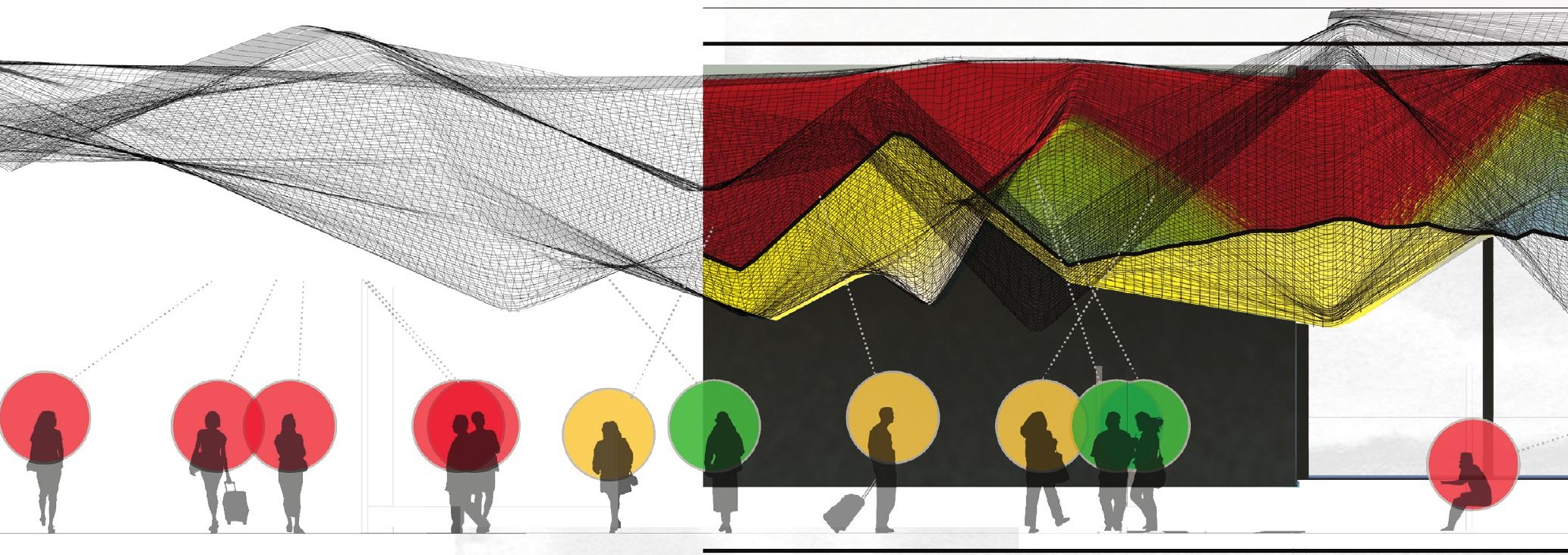

3.71 1:100 section. A collection of users as they move through the developed system. 



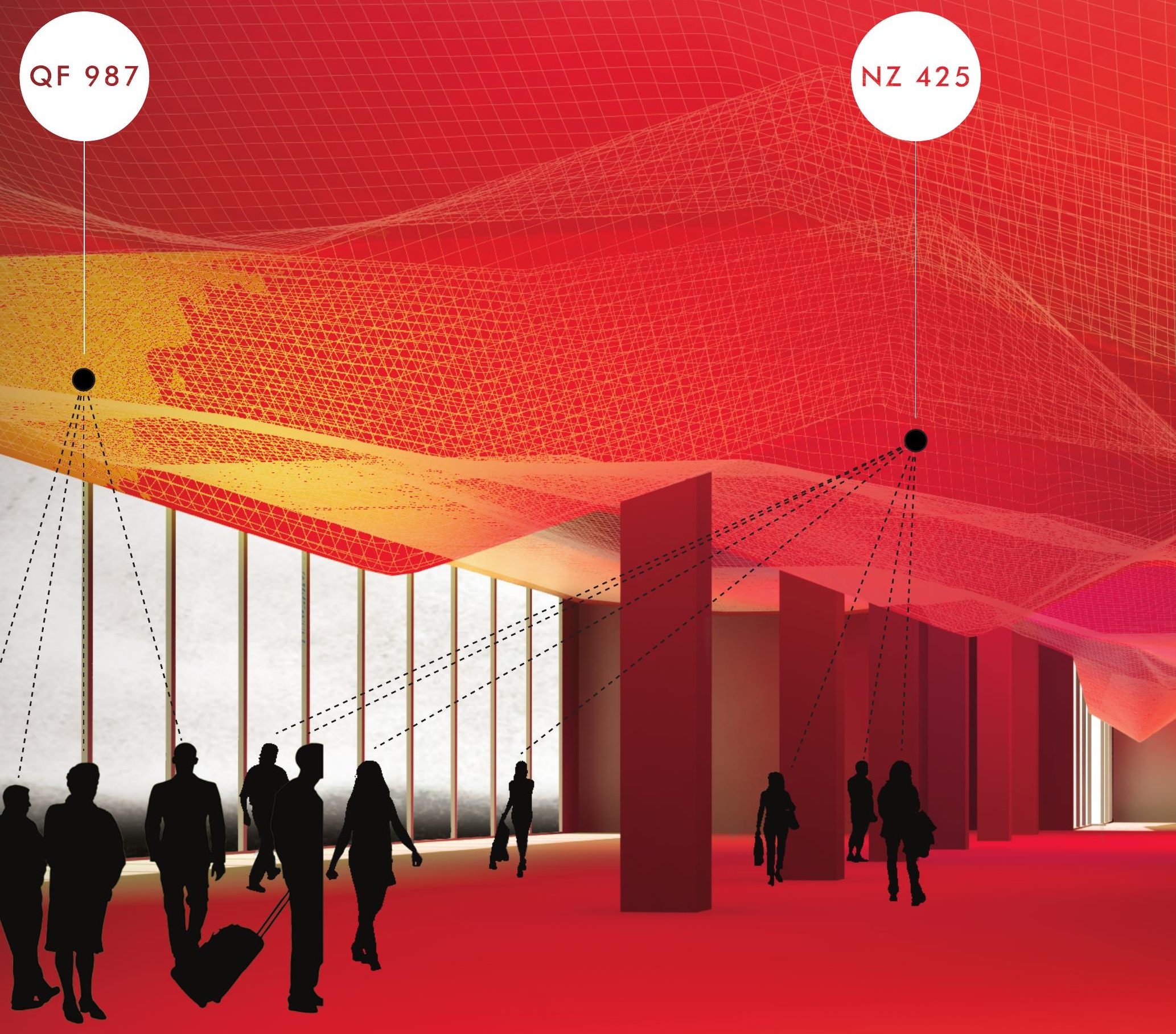

3.72 Concourse Intervention. Users become abstractly represented through their flights. 

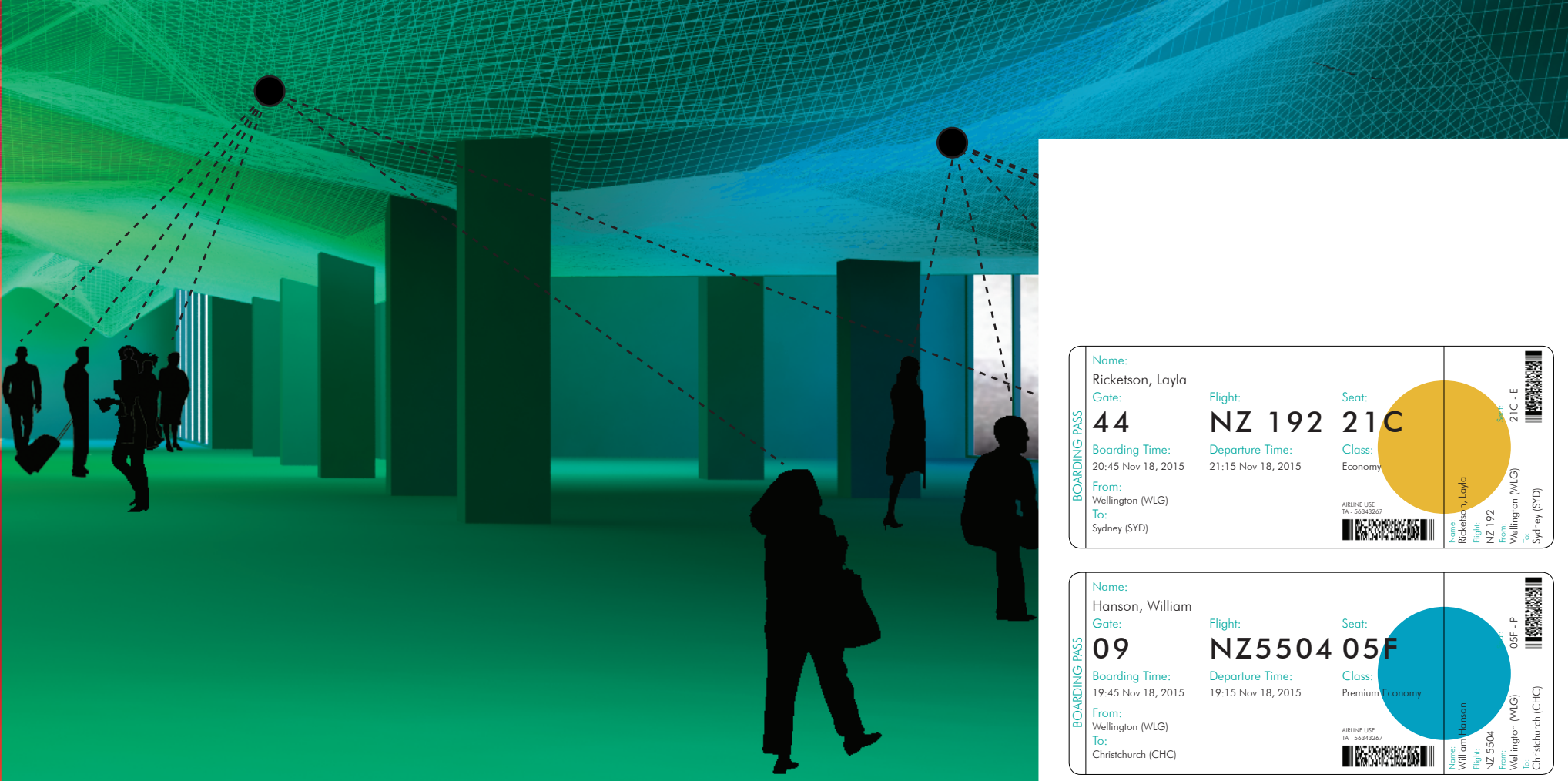

3.73 Boarding passes contain hue information for manual navigation.

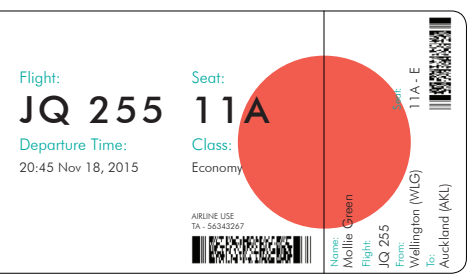




\subsubsection{SYSTEM USAGE}

May 22, 2015

Wellington Airport, South West Pier. $10 \mathrm{am}-11 \mathrm{am}$
3 Departing Flights

NZ 44520 passengers

NZ 27510 passengers

NZ 12418 passengers

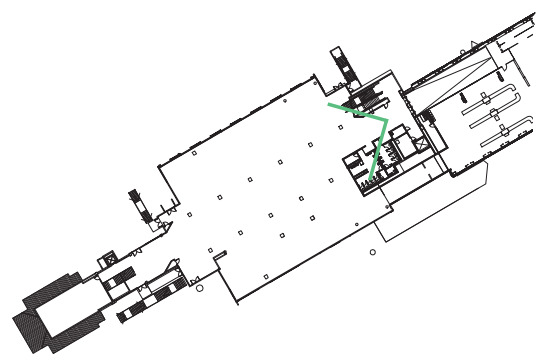

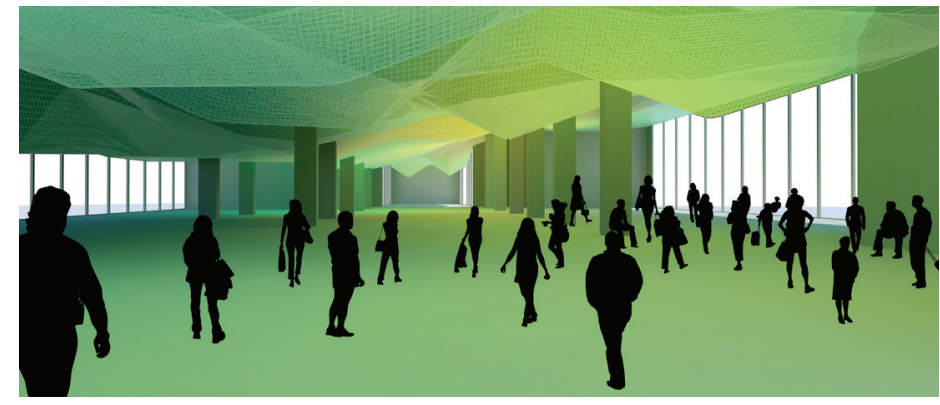
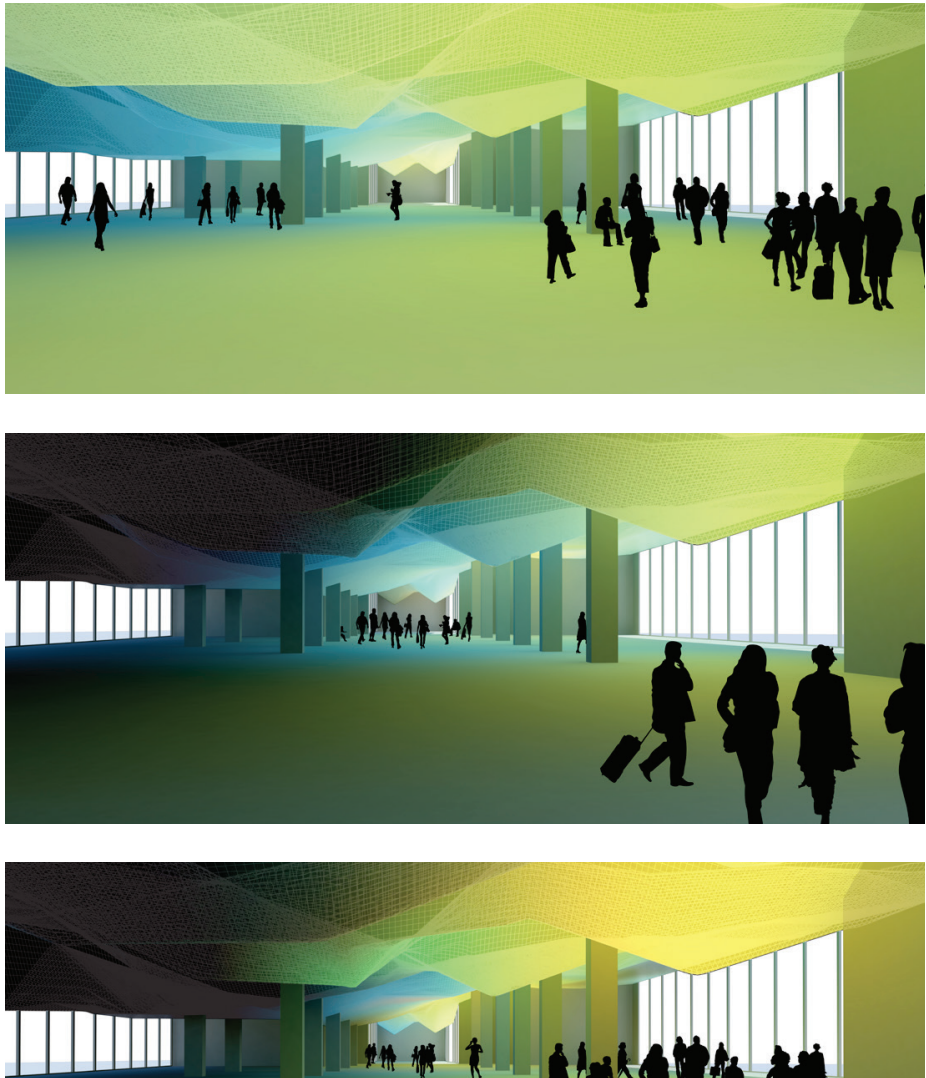
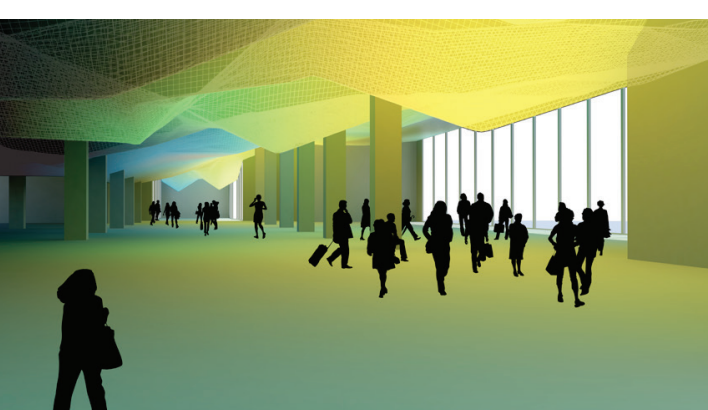

? $10 \mathrm{am}$

NZ 445 Waiting in lounge NZ 275 Arriving in lounge NZ 124 Checking in

$10.15 \mathrm{am}$

NZ 455 Boarding

NZ 275 Waiting in lounge NZ 124 Checking in

$10.30 \mathrm{am}$

NZ 455 Departed

NZ 275 Waiting in lounge NZ 124 Arriving in lounge

$10.45 \mathrm{am}$

NZ 455 Departed NZ 275 Boarding NZ 124 Waiting in Lounge 


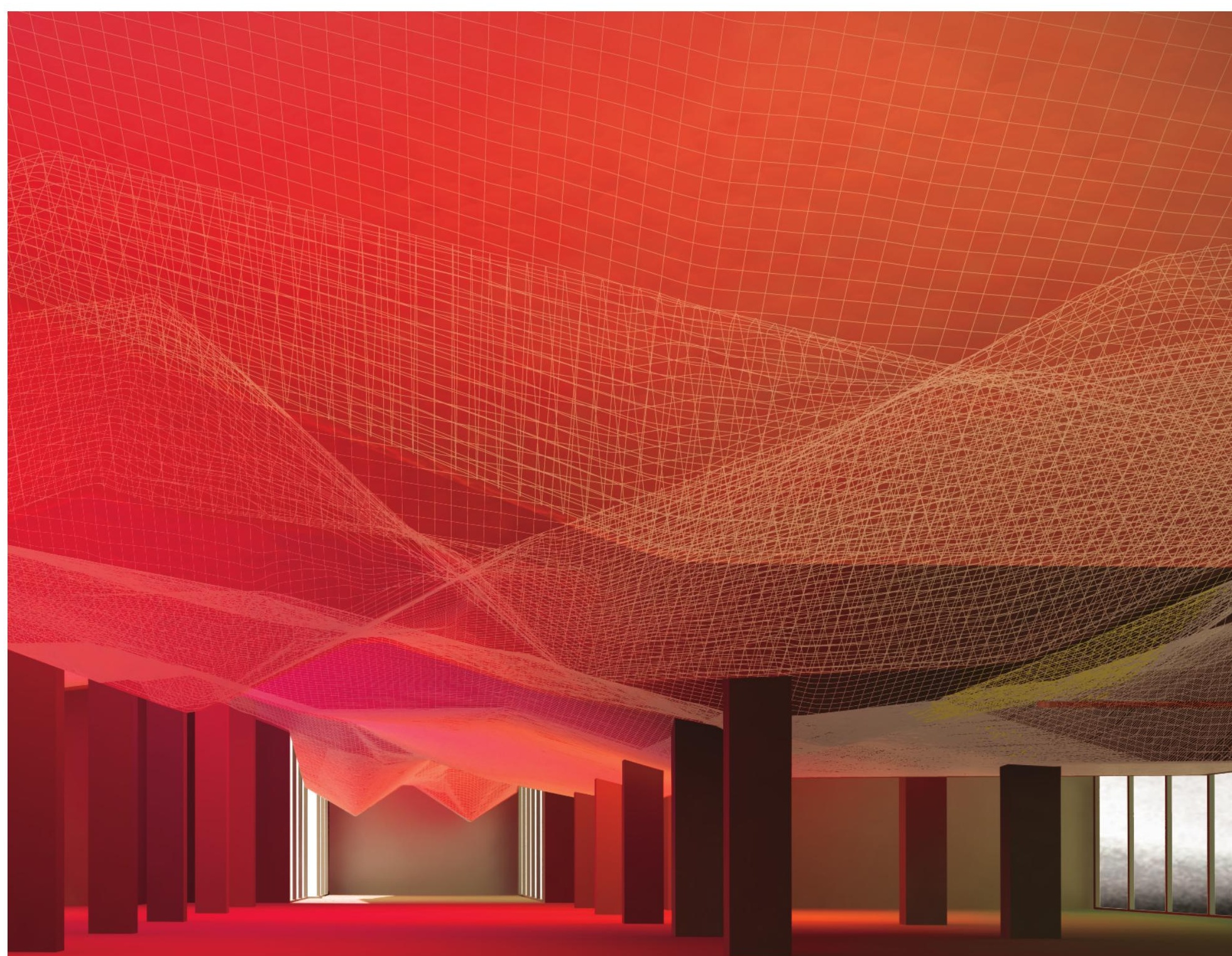

$\leftarrow 3.74$ Although the physical form never changes, the use of the architecture changes the lighting, hence the atmosphere. Areas become highlighted, being responsive to the users at all times.

$\uparrow$ 3.75 Wellington Airport intervention in the south-west pier shows real world use. 
3.76 Design Solution. Virtual-Real space provides real-time way finding system situation within the context of an airport. 
3.77 Users are each mapped to a colour shown across all contexts. 

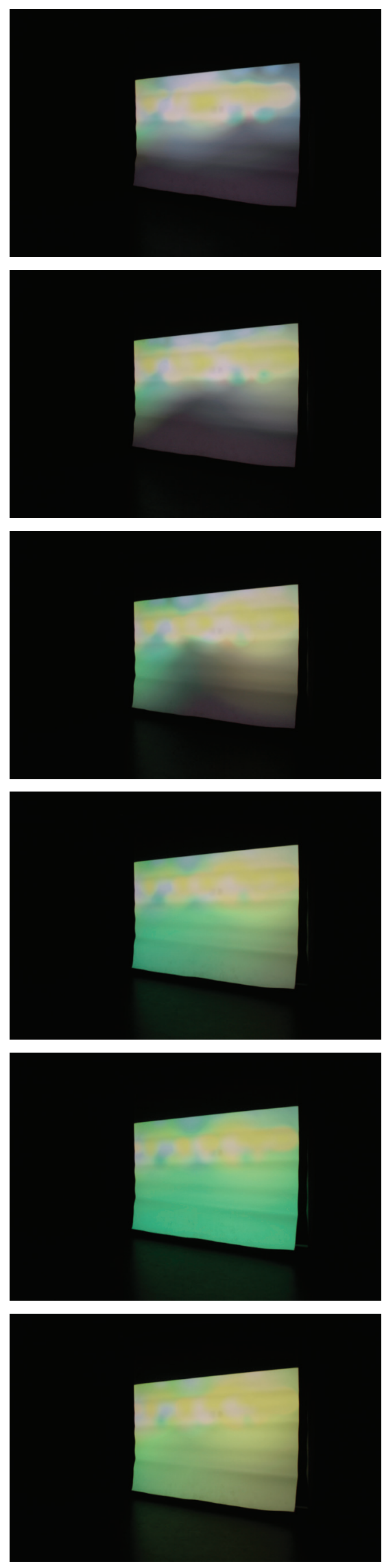

3.78 Time lapse of 1:1 scale projections of visualisation at 30 sec. intervals. Displayed publicly. See appendix A for exhibition photographs. 

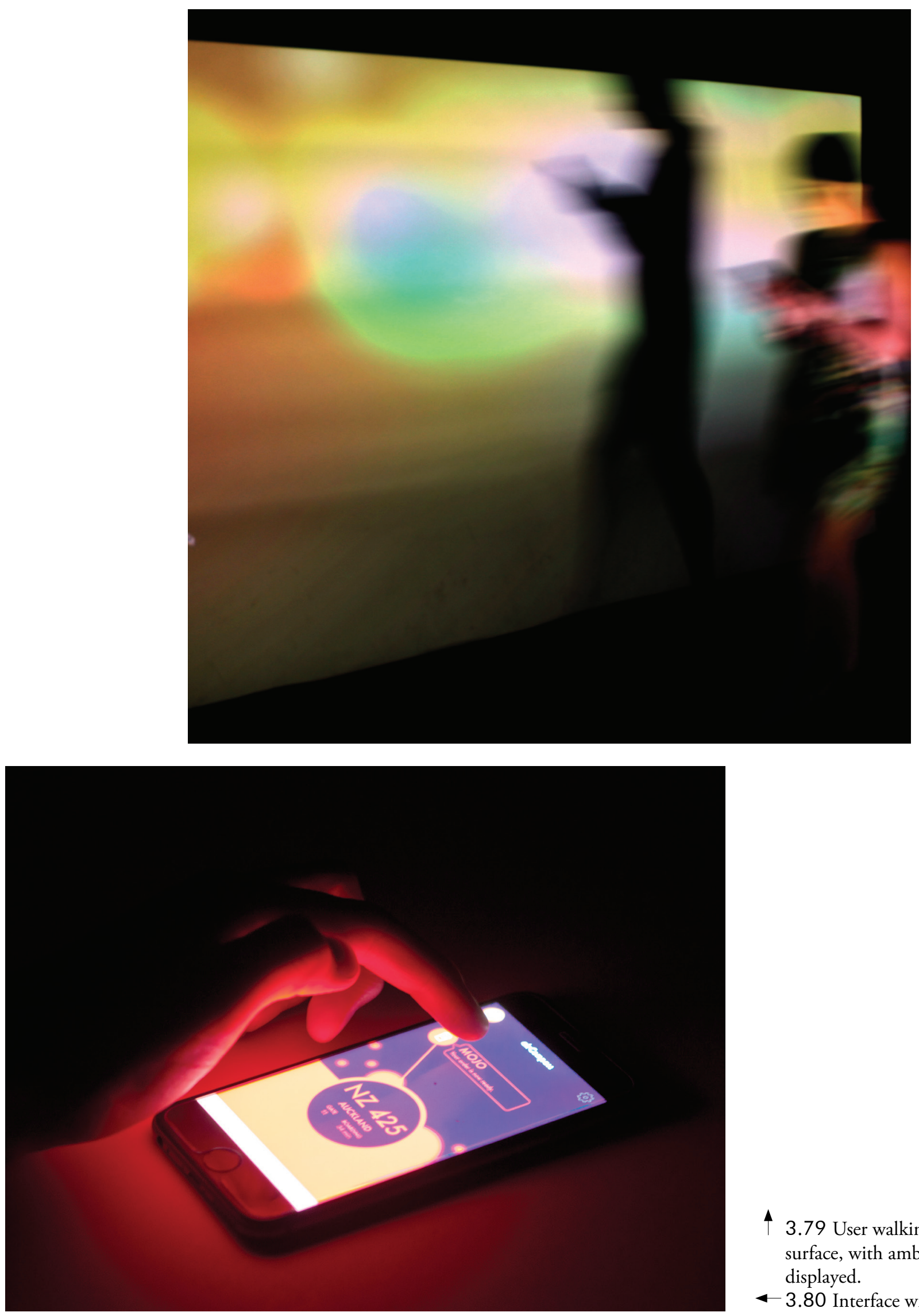

^ 3.79 User walking past the display surface, with ambient information displayed.

$\leftarrow 3.80$ Interface with application. 


\subsection{SUMMARY}

This section devised new ways to experience space through physical and virtual worlds simultaneously. Using the design index, a unified experience to help users to understand data, and other people through data is created. Following this, the project requires further prototyping and testing to develop a fully aware system. The system adherers to understanding of spatial computation, taking computing from a task to a layer of reality with 'a comprehension of physical space which is related to that of the user' (McCullough, 2004).

This section meets many of the MR design index requirements through its use of virtual and real realities to enhance the social and navigational aspects of traditional architecture. The cohesiveness between the visualisation and form highlights this. 


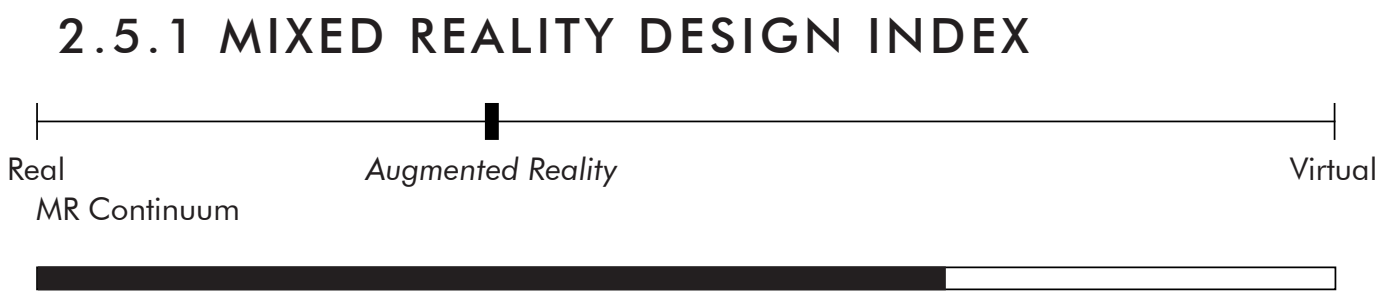

Comprehensive space between material, cognitive and symbolic

Corroboration and reciprocity between physical and virtual components

Extension of a coherent spatial experience and social context.

Anthropic Design

3.81 MR Design Index for second design stage. 


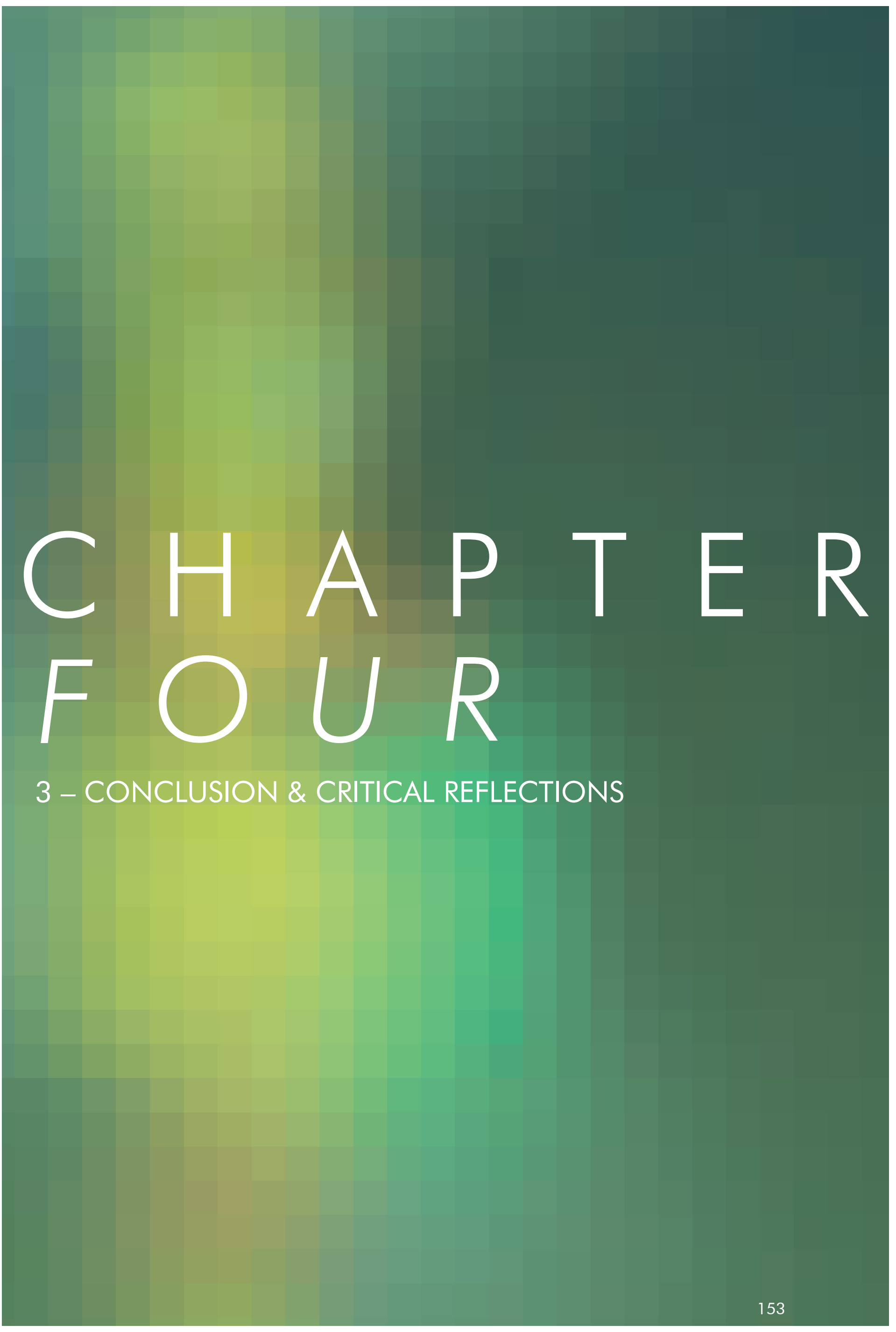




\subsection{CONCLUSION}

This thesis describes the design process of a real-time data influenced way finding system and its use of mixed reality and technologies to spatialise data. Using principles of physical-virtual architecture, a greater level of customised human centric spatial experiences is gained, increasing authenticity, navigation and socialisation. Each stage explored a new set of technologies.

The initial stage uses real time data inputs from API's and humans to provide a base for unique representations in $2 \mathrm{D}$ and $3 \mathrm{D}$. User-centric inputs enables a wide range out outputs to provide depth and layers of information over an iterative process for a $2 \mathrm{D}$ surface. Data sources are then mapped to physical 3D space in real time, providing a kinetic form informed by the flows of users through space, from flight data input of arrivals and departures from Wellington Airport. The stage provided an insight into the spatial ordering of people through designed environments. Accessing against chosen theorists allowed for stage two to be approached in the way it was. These spaces allow for complicated data to become understandable by common users, but overall lacked the personalisation and flexibility that a virtualised system could create.

The second stage involved further translation and processing of this data, mapping it to a virtual representation in real time. Software was written to produce visualisations, which evolved over iterations to understand the location of users in the space, reflecting flows of passengers. Visualisations are displayed on a form originating from data. This way-finding system allows users to understand themselves in the context of other passengers on the same flight and the boarding gate. Mobile application design allows personalised feedback and individual input of locations, which are mapped to points on the visualisation. This iterative effect resulted in a system situated between interactive architecture and mobile navigation systems, highlighting the need for cybrid, dual reality thinking in the development of data-spatial systems.

Personal devices are universal devices, the architecture is a situated device, and this link covers interaction methods based on personal and social preferences. Smartphones are portable and location based, whereas the architecture is responsive, embodied and activity based. This inserts the user within the architecture creation process in real time. By comparing these, their technologies complement each other, the phone is an extension of the human, and the projection is an extension of the architecture.

Pervasive computing should be passive and seamless, this project aims to do this through the use of pre-existing design technologies to create a responsive space that we don't always realise we are a part of. By siphoning off data sources that exist, the system is simply a manifestation of the availability of data in a spatial way.

Embodied interaction aims to create people-to-people relationships through use of using data sources about users in a space, within the space itself, extending social locations. This acts as a motivator to connect humans and improve the use of space. The final experience is an information landscape which users inhabit. The complexity of data sources is reduced to visual, spatial and form related communications which is aware of its environment and is responsive to 
users. Uncovering hidden patterns from data occurs.

This research raises questions about data selection in a preserved physical form, indicating that shape changing architectural devices may be the next avenue to explore. It also asks how the human use of space might change when systems such the one designed, become pervasive and has the ability to become personal and customised. The design process enhanced the possible connection that can be made between technology systems and architectural experience, not just to create high a performance building, but a building that perform socially around the user. 


\subsection{NEXT STEPS}

\subsubsection{TECHNICAL}

As the project is a prototype, there remain issues to be further resolved. This includes a self-adjusting mechanism for delays and changes to flight times and adding Bluetooth beacons and IPS technology into the software.

Data transformed into physical forms raises questions about which data is static, becoming physical space, and which data is dynamic, used in the projection. Static data chosen changes periodically, so should possibly use natural and consistent inputs, such as the exact location of the architecture. Alternatively, not using data at all, instead an abstract interpretation of spatial qualities could be used.

There needs to be a better understanding how the projections will work. The addition of LCD panels in forms, or LED lighting will allow it to happen, and could be prototyped. Furthermore, the size and shape of airports will have to be considered. The architectural projection accounts for some of this, however the overall simulation needs a method for understanding boundaries of space.

\subsubsection{SPATIAL}

The use of various IoT sensors could be included, as currently there is input from users with both physical and virtual interfaces. Virtual data about the space is included, however data pulled physically is not. Some of the case studies use sensors as a design tool, and could be used in the project to alter the virtual visualisation in real time to the context, however this is already done to a degree with human location inputs.

There are possibilities for the spatial system to act a design tool for the layout and organisation of airports. Data allows flights to be grouped in different ways, for example by destination; information architecture though data. Simulations of the space can also inform the shape and layout, for example how people flow through space to create circulation space? 


\subsection{LIMITATIONS}

A universal language allows the system to scale to various architectural elements as needed. Allowing a greater number of flights to be seen on the system at one time without effecting the usability and clarity of the design language is a limitation. Through awareness of location, the software can determine the best set of colors for the given users. Future work could also look at methods of sorting passengers into gate lounges, then flights after that using a different set of hues.

In reality, the installed system would require anonymity and privacy to be reassured. As the key human data inputs are flight arrivals and departures, and human location from app enabled personal devices, users should be aware their data is being used.

In future steps, a fuller understanding of the link between the projection and form will need to be resolved. This may end up in more iterations of the form or visual language. Ensuring these are closely related will benefit the integration humans. 


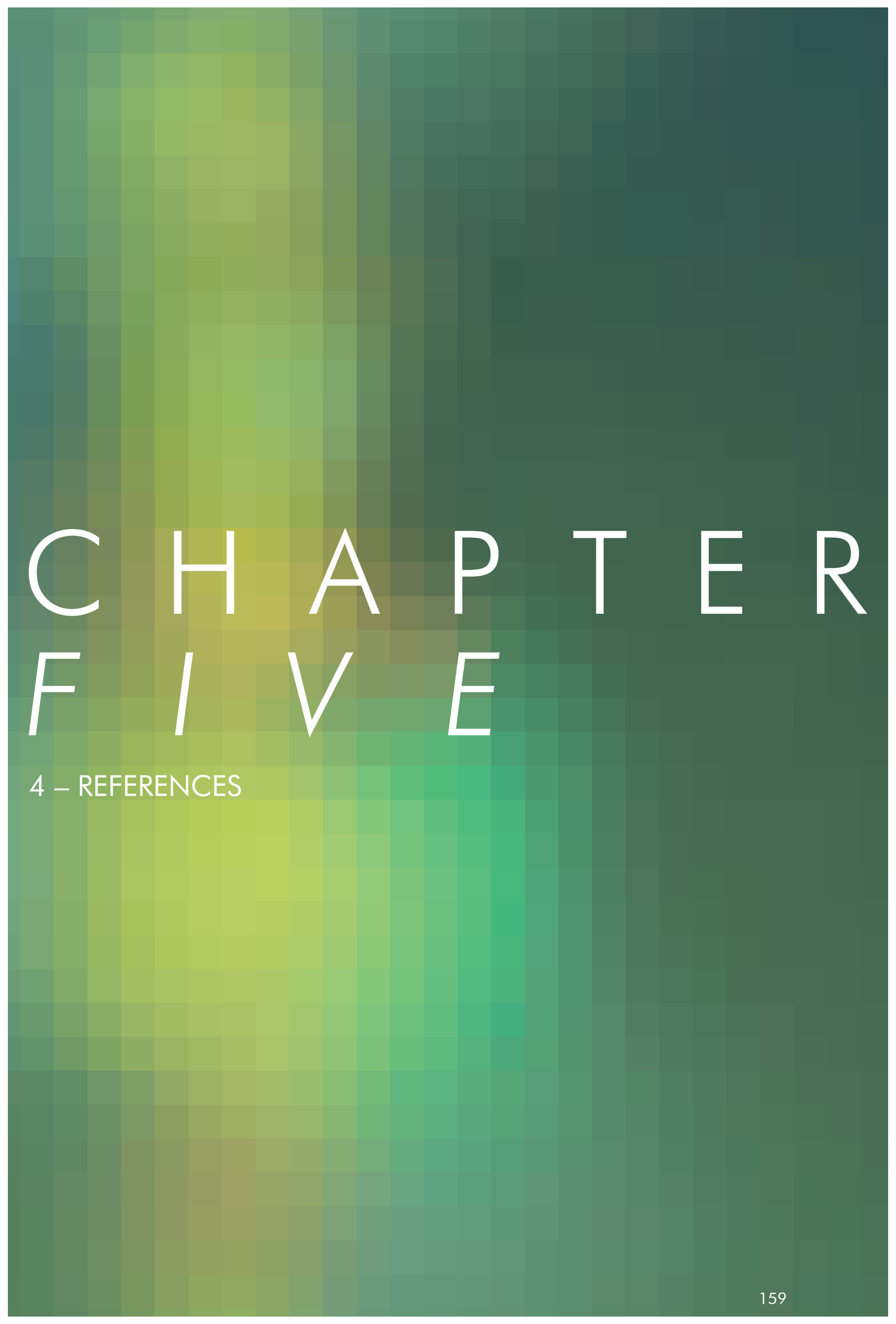




\section{1 REFERENCES}

Achten, H. (1997). Generic Representations - Typical Design without the Use of Types. In CAAD Futures 1997 (pp. 117-133). München (Germany). Retrieved from http://cumincad.scix.net/

Achten, H. (2015). Closing the Loop for Interactive Architecture - Internet of Things, Cloud Computing, and Wearables. In Real Time - Proceedings of the 33rd eCAADe Conference (Vol. 2, pp. 623-632). Vienna University of Technology, Vienna, Austria. Retrieved from http://cumincad.scix.net/

Anders, P. (2007). Designing Mixed Reality: Principles, Projects and Practice. In Expanding Bodies: Art ? Cities? Environment - Proceedings of the 27th Annual Conference of the Association for Computer Aided Design in Architecture (pp. 276-283). Halifax (Nova Scotia).

Cooper, A. (2007). About face 3 the essentials of interaction design. Indianapolis, IN: Wiley Pub.

CreativeApplications. net. (2014). Holo 1 : Emerging trajectories in art, science and technology. Toronto: CreativeApplicationsnet.

Dourish, P. (2001). Where the Action Is. Cambridge MA: MIT Press. Retrieved from https://mitpress.mit.edu/books/where-action

Downton, P. (2003). Design research. Melbourne: RMIT Publishing.

Estimote. (n.d.). Retrieved January 31, 2016, from http://estimote.com/

Fry, B. (2008). Visualizing data. Sebastopol, CA: O’Reilly Media, Inc.

Hansen, K. and M. (2015). Designing Real Time Sense and Response Environments through UX Research. In Real Time - Proceedings of the 33rd eCAADe Conference (Vol. 2, pp. 651-658). Vienna University of Technology, Vienna, Austria. Retrieved from http://cumincad.scix.net/

Herzog \& de Meuron. (n.d.). Allianz Areana. Retrieved January 31, 2016, from https://www.herzogdemeuron.com/index/projects/complete-works/201225/205-allianz-arena.html

Infratil - Airports. (2015). Wellington Airport 2015 Results and Annual Report. Retrieved February 11, 2016, from http://www.infratil.com/infratilnews/2015/wellington-airport/

Izaki, Å., \& Helme, L. (2014). Encoding User Experiences. Architectural Design, 84(5), 114-121. http://doi.org/10.1002/ad.1817

Kuo, C.-G., Lin, H.-C., Shen, Y.-T., \& Jeng, T.-S. (2004). Mobile Augmented Reality for Spatial Information Exploration. In CAADRIA 2004 - Proceedings of the 9th International Conference on Computer Aided Architectural Design Research in Asia (pp. 891-900). Seoul, Korea. Retrieved from http://cumincad.scix.net/ 
McCullough, M. (2004). Digital ground : architecture, pervasive computing, and environmental knowing. Cambridge, Mass: MIT Press.

Milgram, P., \& Kishino, F. (1994). A taxonomy of mixed reality visual displays. IEICE TRANSACTIONS on Information and Systems, 77(12), 1321-1329.

Moloney, J. (2011). Designing Kinetics for Architectural Facades : State Change (1st ed.). Hoboken: Taylor and Francis.

Motion Capture Software and Mocap Tracking Info. (2015). Retrieved December 8, 2015, from http://www.organicmotion.com/motion-capture/

Processing.org. (n.d.). Retrieved January 31, 2016, from https://processing. org/

Reynolds, C. (2015). Boids (Flocks, Herds, and Schools: a Distributed Behavioral Model). Retrieved November 21, 2015, from http://www.red3d. com/cwr/boids/

Schnabel, M. A., Wang, X., Seichter, H., \& Kvan, T. (2007). From Virtuality to Reality and Back. In International Association of Societies of Design Research 2007 (IASDR07). The Hong Kong Polytechnic University Hung Hom, Kowloon, Hong Kong. Retrieved from http://cumincad.scix.net/

Segard, A., Moloney, J., \& Moleta, T. (2013). Open Communitition Competitive Design in a Collaborative Virtual Environment. In Open Systems: Proceedings of the 18th International Conference on ComputerAided Architectural Design Research in Asia (CAADRIA 2013) (pp. 231240). Singapore. Retrieved from http://cumincad.scix.net/

Shekhar, S., Feiner, S. K., \& Aref, W. G. (2015). Spatial computing. Communications of the ACM, 59(1), 72-81. http://doi. org/10.1145/2756547

Vertegaal, R., \& Poupyrev, I. (2008). Introduction. Communications of the ACM, 51(6), 26. http://doi.org/10.1145/1349026.1349033

Wu, C., \& Clayton, M. J. (2015). Visualizing Climate Data as a 3D Climate Torus. Retrieved September 8, 2015, from http://cumincad.scix.net/ 


\subsection{SOURCE OF FIGURES}

Note: All figures not attributed are author's own.

Figure 1.01: Aaron Koblin, Design Media Arts, UCLA. Flight Paths, 2005 $<$ http://users.design.ucla.edu/ akoblin/work/faa/>

Figure 1.06: Breakfast. Points Sign. 2013. New York, NY. <https:// pointssign.com/static/img/promosite/action/vid_bg.jpg>

Figure 1.07: Breakfast. Points Sign, 2013. New York, NY. <http:// d3uifzcxlzuvqz.cloudfront.net/images/stories/content/products/points/pointssign-rotation.jpg>

Figure 1.09: ONL Architecture. Salt Water Pavilion, 1997. Neeltje Jans, Netherlands. <http://vaa.onl/projects/salt-water-pavilion>

Figure 1.10: ONL Architecture. Salt Water Pavilion, 1997. Neeltje Jans, Netherlands. <http://vaa.onl/projects/salt-water-pavilion>

Figure 1.12: Echelman, Janet \& Aaron Koblin. Unnumbered Sparks, 2014. Vancouver, Canada. <http://www.unnumberedsparks.com/images/index/ gallery-01.jpg>

Figure 1.13: Echelman, Janet \& Aaron Koblin. Unnumbered Sparks, 2014. Vancouver, Canada. <https://tedconfblog.files.wordpress.com/2014/03/1323 7626894_744c2672a4_h.jpg>

Figure 1.15: Herzog and de Meuron. Allianz Arena, 2005. MünchenFröttmaning, Germany. <http://www.meisterhermann.ro/wp-content/ uploads/2015/10/Allianz_Arena5.jpg>

Figure 1.16: Herzog and de Meuron. Allianz Arena, 2005. MünchenFröttmaning, Germany. <https://sites.google.com/site/deutschindieschule/_/ rsrc/1430732735925/home/Allianz_arena_golden_hour_Richard_Bartz.jpg>

Figure 1.18: Phillip Beesley Architect Inc. Hyzolic Ground, 2010.

Canadian Pavilion, Venice Biennale. <http://philipbeesleyarchitect.com/ sculptures/0929_Hylozoic_Ground_Venice/PBAI_03.jpg>

Figure 1.19: Phillip Beesley Architect Inc. Hyzolic Ground, 2010. Canadian Pavilion, Venice Biennale. <http://philipbeesleyarchitect.com/ sculptures/0929_Hylozoic_Ground_Venice/PBAI_09.jpg>

Figure 1.21: doubleNegatives Architecture. Corpora in Si(gh)te, 2008. Yamaguchi, Japan. <http://special.ycam.jp/corpora/img/top_img.jpg>

Figure 1.22: doubleNegatives Architecture. Corpora in Si(gh)te, 2008. Yamaguchi, Japan. <http://special.ycam.jp/corpora/img/top_img.jpg>

Figure 2.02: Wellington City Council. $5 \mathrm{~m}$ contours \& building footprints. 2004 \& 2015. Wellington, New Zealand. <https://koordinates.com/> 
Figure 2.09: MIT Media Lab. inFROM, 2013. <http://www. creativeapplications.net/openframeworks/inform-dynamic-shape-displayfrom-tangible-media-group/>

Figure 3.06: Princemio. Pathfinder, 2013 <http://www.creativeapplications. net/wp-content/uploads/2014/11/pathfinder_photos_06.jpg>

Figure 3.56: Harlan, Dev. Parmenides, 2011. New York, NY 10013, United States < http://www.arch2o.com/astral-flight-hangar-christopher-henry/>

Figure 3.64: Vijgen, Richard. The Architecture of Radio, 2015. < http:// www.creativeapplications.net/javascript-2/three-js/the-architecture-of-radiovisualizing-the-invisible-architecture-of-networks/> 


\subsection{APPENDICIES}

APPENDIX A: PUBLIC DISPLAY OF WORK IN INTERIOR ARCHITECTURE EXIBITION.

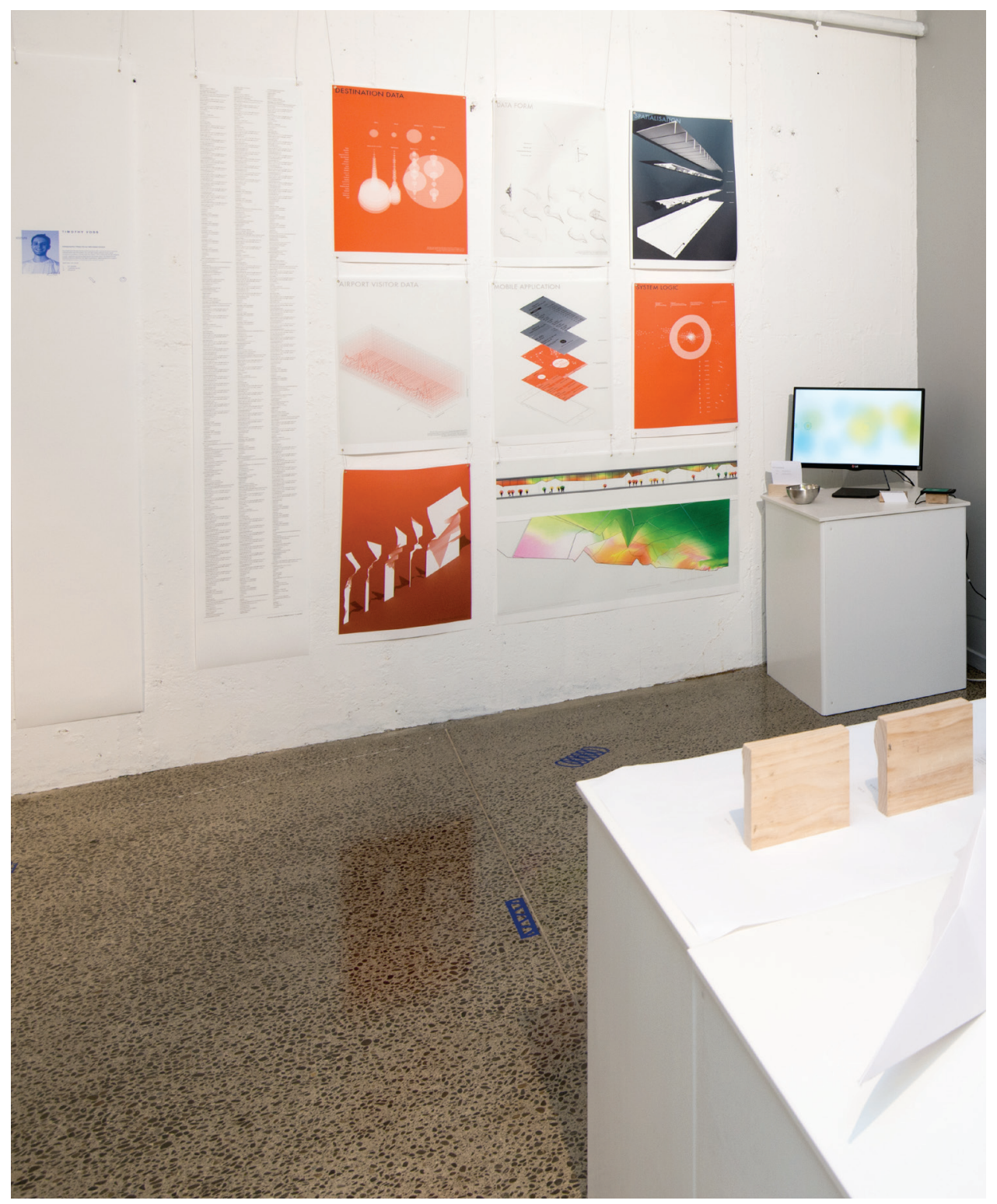




\section{APPENDIX B: TECHNOLOGY TESTS.}

\section{Technology Test 01}

Kinect Skeleton - Real Time

The skeleton test using the firefly plug in for grasshopper allows points on a person to be tracked in real time. This plug in also allows an output of lines between points. The test involved understanding how humans can affect a form.

Pros

- Accurate point tracking of human movement

- Directly into rhino

- Easy to set up and link to existing models and logic

- Real time

Cons

- Slow at updating logic based on tracking

- Can't save particular states

Next:

- How to store frames/sequences

- Quicker responding forms

\section{Technology Test 02}

Kinect Point Cloud - Real Time

The point cloud test using the firefly plug in for grasshopper allows the depth field to be translated to points in real time and displayed in rhino/grasshopper. This records the entire space the sensor seeing, including the space, so is better for an overall idea of the space. It also allows for distinct human features such as size, clothes, facial features etc. to be recorded.

Pros

- Easy to set up

- Real time

- Adjustable resolution

- Directly into rhino

Cons

- Slow at updating in real time - grasshopper is prone to crashing

- No recording feature

- No separation of foreground and background

Next

- Store frames/sequences

- Understand how best to use it to dictate form

\section{Technology Test 03}

Kinect Point Cloud - Recorded

Brekel Pro Point Cloud v1 for Kinect was used to record the points. This software has a large amount of options allowing for various amounts of points 
to be exported. Data is accurate and recorded quickly at $30 \mathrm{fps}$.

Pros

- Fast saving for later use in design

- Export to large amount of formats including .obj and .bin (real flow)

- Saves .jpg texture maps

- Has skeleton tracking version

Cons

- No real time output

- Involves in depth processing to get animation recording into Rhino or 3Ds Max

Next:

- Points based around the movement - rendered out?

\section{Technology Test 04}

Real Time Weather Input

Using a plug in for grasshopper, gHowl, XML feeds from the internet can be inputted into logic. Currently I am using openweathermaps.org to pull data. A large variety of data sources can be gathered, such as temperature, humidity, exact location, precipitation and wind speed and direction. This is outputted in a numerical format.

Pros

- Easy to input new location

- accurate and real time from internet

Cons

- need to enable/disable component to refresh

- XML data is difficult to parse in grasshopper (large data sets)

Next:

- How get multiple data points across multiple locations

- Self-refreshing (timer in grasshopper for input possibly)

\section{Technology Test 05}

Real Time Flight Tracking Input

Using the same gHowl plug in for grasshopper, the data can be retrieved about arrival and departure information from any given airport. Using API's from flightstats.com the information can be downloaded in real time, for six hour intervals.

Pros

- Easy to change data request inputs

- Accurate data is being provided

Cons

- need to enable/disable component to refresh

- XML data is difficult to parse in grasshopper (large data sets) and there is various tiers to each set of data (e.g. each flight has an airline code, number, 
time and gate)

- Can get better formatted information through excel

Next:

- Find a way to parse the information better and make it usable for design

- Self-refreshing (timer in grasshopper for input possibly)

- Find out which script will do this best

\section{Technology Test 06}

Android/ mobile phone integration

Using the android mode of processing, it was possible to get the sketch used in the previous design stage working. This then allows the implementation of location based feedback and interaction through the touch and movement sensors on the device.

\section{Technology Test 07}

Social Network inputs

Using twitter feeds, take the recent tweets about a particular airport and create a cloud of words. This cloud of words, sentences and images could then create a texture, collage or interaction point for people. 
- end - 
